\title{
New horizons in lymph node imaging in head and neck cancer
}

Citation for published version (APA):

de Bondt, R. B. J. (2010). New horizons in lymph node imaging in head and neck cancer. [Doctoral Thesis, Maastricht University]. Datawyse / Universitaire Pers Maastricht.

https://doi.org/10.26481/dis.20101215rb

Document status and date:

Published: 01/01/2010

DOI:

10.26481/dis.20101215rb

Document Version:

Publisher's PDF, also known as Version of record

\section{Please check the document version of this publication:}

- A submitted manuscript is the version of the article upon submission and before peer-review. There can be important differences between the submitted version and the official published version of record.

People interested in the research are advised to contact the author for the final version of the publication, or visit the DOI to the publisher's website.

- The final author version and the galley proof are versions of the publication after peer review.

- The final published version features the final layout of the paper including the volume, issue and page numbers.

Link to publication

\footnotetext{
General rights rights.

- You may freely distribute the URL identifying the publication in the public portal. please follow below link for the End User Agreement:

www.umlib.nl/taverne-license

Take down policy

If you believe that this document breaches copyright please contact us at:

repository@maastrichtuniversity.nl

providing details and we will investigate your claim.
}

Copyright and moral rights for the publications made accessible in the public portal are retained by the authors and/or other copyright owners and it is a condition of accessing publications that users recognise and abide by the legal requirements associated with these

- Users may download and print one copy of any publication from the public portal for the purpose of private study or research.

- You may not further distribute the material or use it for any profit-making activity or commercial gain

If the publication is distributed under the terms of Article $25 \mathrm{fa}$ of the Dutch Copyright Act, indicated by the "Taverne" license above, 


\title{
NEW HORIZONS IN LYMPH NODE IMAGING IN HEAD AND NECK CANCER
}

\author{
R.B.J. de Bondt
}


ISBN 9789461590152

(C) Copyright RBJ de Bondt, Maastricht 2010

Print by Datawyse / Universitaire Pers Maastricht

Financial support of this thesis by: Bayer-Shering-Pharma, Philips Medical Systems, Hagamedical B.V., Tromp B.V. and Agfa Healthcare. 


\title{
NEW HORIZONS IN LYMPH NODE IMAGING IN HEAD AND NECK CANCER
}

\author{
PROEFSCHRIFT \\ ter verkrijging van de graad van doctor \\ aan de Universiteit Maastricht, \\ op gezag van de Rector Magnificus, \\ Prof. Mr. G.P.M.F. Mols, \\ volgens het besluit van het College van Decanen, \\ in het openbaar te verdedigen \\ op woensdag 15 december 2010 om 16.00 uur \\ door
}

Bert Jan de Bondt

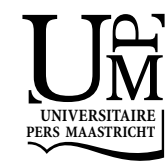




\section{Promotoren}

Prof. dr. R.G.H. Beets-Tan

Prof. dr. B. Kremer

\section{Copromotoren}

Dr. J.W. Casselman

Dr. P.J. Nelemans

\section{Beoordelingscommissie}

Prof. dr. C. Boetes (voorzitter)

Prof. dr. R.J. Baatenburg de Jong

Prof. dr. J.O. Barentsz

Prof. dr. J.A. Castelijns

Prof. dr. P. Kessler

Prof. dr. Ph. Lambin

\section{Paranimfen}

Dennis den Hartog

Maarten Boot 
Aan

Margreet, Sophie,Marijn en Merel

Ruud en Lineke

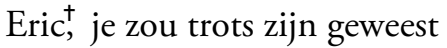


$\longrightarrow$ 


\section{Contents}

Chapter 1 General introduction and outline of this thesis 9

Chapter 2 Lymph node imaging in the head and neck: overview of the 17 present status of modern imaging techniques

Chapter 3 Detection of lymph node metastases in head and neck cancer: A meta-analysis comparing US, USgFNAC, CT and MR imaging

Chapter 4 Clinical impact of STIR MRI on staging and management in 43 patients with cervical lymph node metastases of head and neck squamous cell carcinomas.

Chapter 5 Morphological MRI criteria improve the detection of lymph 59 node metastases in head and neck squamous cell carcinoma: multivariate logistic regression analysis of MRI features of cervical lymph nodes

Chapter 6 Diagnostic accuracy and additional value of diffusion weighted 77 imaging for discrimination of malignant cervical lymph nodes in head and neck squamous cell carcinoma.

Chapter 7 General discussion and future perspectives

$\begin{array}{lll}\text { Chapter } 8 \text { Conclusions and recommendations } & 105\end{array}$

$\begin{array}{lll}\text { Chapter } 9 \text { Summary } & 109\end{array}$

$\begin{array}{lll}\text { Chapter } 10 & \text { Samenvatting } & 113\end{array}$

$\begin{array}{ll}\text { Dankwoord } & 117\end{array}$

Curriculum Vitae $\quad 121$

List of publications $\quad 122$ 
$\longrightarrow$ 


\section{CHAPTER 1}

General introduction and outline of this thesis 
$\longrightarrow$ 


\section{Primary tumor and tumor spread}

Head and neck squamous cell carcinomas (HNSCC) originate from the mucosal lining of the upper aero digestive tract. Squamous cell carcinoma is the most frequently occurring histological type of tumor in the head and neck as it accounts for almost $90 \%$ of all head and neck cancers. Important risk factors are cigarette smoking and alcohol abuse; especially, the combined use of both is related to an increased risk to develop a head and neck carcinoma. Recently, a new group of HNSCC caused by oncogenic human papilloma virus (HPV) has been identified [10]

The pattern of regional tumor spread is predominantly through the lymphatic system to the cervical lymph nodes, rather than haematogenous dissemination. Distant metastases will occur almost exclusively after the tumor has spread to the cervical lymph nodes. The probability that cervical lymph node metastases are present at diagnosis is closely correlated to the etiology, location, stage and histology of the primary tumor.

Nodal tumor spread is the most important prognostic factor in HNSCC. Therefore, the choice of work up and management highly depends on the extent of lymph node metastases, which not only means the presence but also the number and distribution of lymph node metastases in the different levels of the neck. Such a nodal staging can guide decisions whether radical treatment involving elective dissection of lymph nodes is necessary or whether more conservative treatment options such as targeted radiotherapy, minimal invasive surgery such as laser surgery, and adequate use of combined neoadjuvant therapy suffice. For this reason, imaging modalities which are able to visualize the extent of lymph node metastases are a crucial part of the diagnostic work-up in patients who present with HNSCC. Accurate radiological imaging staging of head and neck cancers together with clinical staging can lead to a tailored treatment strategy without any compromise to the local control.

\section{Management of the clinically negative (N0) and positive $\left(\mathrm{N}_{+}\right)$neck}

A major challenge is clinical decision making with respect to treatment of the clinically negative neck. The clinically negative (N0) neck is defined as a neck without palpable pathological lymph nodes. One treatment option is to follow a so-called "wait-and-see policy", where it is decided to refrain from elective dissection of lymph nodes. The advantage of this approach is that unnecessary 
morbidity due to surgery can be avoided. However, due to the inability to completely rule out the presence of lymph node metastases by means of palpation, there is a risk that occult metastases remain undetected and that the patient is undertreated. There is serious concern that salvage of occult metastases that become manifest during follow-up is much less effective than elective prophylactic removal shortly after diagnosis and treatment of the primary tumor. For this reason, more aggressive treatment including elective prophylactic neck dissection, radiotherapy or a combination of both therapies are alternative options for treatment. In current practice, this is solved by deciding to refrain from a "waitand-see policy" when the estimated risk of occult metastases exceeds 20\% [7] This estimate is based on location, stage and histology of the primary tumor. However, more aggressive approaches may result in overtreatment because this chosen cut-off point is rather arbitrarily and the risk estimate may be unreliable. For these reasons, treatment of the clinically negative neck remains controversial.

In the clinically positive $\left(\mathrm{N}_{+}\right)$neck pathological lymph nodes are palpable and neck dissection, in which lymph nodes would be removed, radiation therapy, or a combination of both are indicated. Extent and distribution of lymph node metastases will again determine the choice of therapy.

\section{Radiological imaging modalities}

Hence, in patients with HNSCC, accurate staging of the extent of lymph node metastases in $\mathrm{N} 0$ and $\mathrm{N}+$ necks is of crucial importance in order to help the clinician to make a confident and accurate diagnosis and to decide on the optimal treatment of individual patients. Accurate prediction of lymph node involvement is not possible with the present imaging modalities, unless combined with invasive and demanding procedures such as ultrasound guided fine needle aspiration cytology (USgFNAC). Besides the radiological imaging modalities, such as US, CT and MRI, there is an upcoming role of 18 F-FDG PET for the detection of cervical lymph node metastases. However, this role is limited for small metastases [9; 9; 2; 9; 5; 5]. The use of the integrated PET-CT as a one step examination is in literature advocated to be the initial imaging modality for advanced staged HNSCC. Again, the limitation is the detection of small lymph node metastases. [7; $11 ; 12 ; 9 ; 12 ; 4 ; 2 ; 10 ; 11]$

Assessment of lymph nodes using imaging is mainly based on size, but the mere use of the size criterion has several disadvantages. First, it is difficult to establish an optimal threshold value. The use of common threshold values, such as $\leq 7 \mathrm{~mm}$ or 
$\leq 10 \mathrm{~mm}$ result in suboptimal sensitivity and lowering of the cut-off point results in higher sensitivity, but consequently also in lower specificity [2]. Second, metastases in small cervical lymph nodes will remain below the threshold levels and consequently these malignant lymph nodes will be misclassified as normal. Therefore, additional criteria have to be established to improve diagnostic accuracy.

\section{Objectives of this thesis}

This thesis will focus on the diagnostic performance of MRI and the main aim will be to investigate and improve the diagnostic accuracy of MRI for the differentiation of malignant from benign lymph nodes in HNSCC.

The objectives of this thesis are:

- To evaluate the diagnostic value of MRI relative to that of other methods that are used for the detection of cervical lymph node metastases in patients with HNSCC.

- To evaluate whether the use of new sequences and/or other MRI criteria in addition to size can improve accurate detection of metastatic lymph node disease in patients with HNSCC.

\section{Outline of this thesis}

First an overview is given of all applicable imaging modalities in which for every modality the present diagnostic accuracy is discussed (chapter 2). In order to further compare the diagnostic accuracies of the presently used radiological imaging modalities a meta-analysis is presented in which ultrasound, USgFNAC, CT and MR imaging are compared (chapter 3). The use of the STIR sequence is investigated in order to evaluate the added diagnostic value over conventional MRI for the detection of cervical lymph nodes metastases (chapter 4). The accuracy and additional diagnostic value of different morphological criteria is assessed by means of multivariate logistic regression analysis. It is evaluated whether use of these criteria in addition to size results in better prediction of the presence of metastasis in cervical lymph nodes (chapter 5). Subsequently, the diffusion weighted imaging (DWI) sequence was assessed. Use of this sequence provides information on apparent diffusion coefficient (ADC) of different tissues and lesions. The diagnostic accuracy and additional value of the ADC value - compared to the size and morphological criteria - is assessed by means of univariate and multivariate 
logistic regression analyses. Evaluation was performed in a series of patients with predominantly clinically negative necks (chapter 6).

Finally, the results of the various studies are discussed and suggestions for future directions will be made (chapter 7). A summary is provided in chapter 8 . 


\section{References}

1. Adams, S., Baum, R. P., Stuckensen, T., et al., "Prospective comparison of 18F-FDG PET with conventional imaging modalities (CT, MRI, US) in lymph node staging of head and neck cancer." Eur J Nucl Med. 1998. 25(9): 1255-60.

2. Brink, I., Klenzner, T., Krause, T., et al., "Lymph node staging in extracranial head and neck cancer with FDG PET--appropriate uptake period and size-dependence of the results." Nuklearmedizin. 2002. 41(2): 108-13.

3. Brouwer, J., de Bree, R., Comans, E. F., et al., "Positron emission tomography using [18F]fluorodeoxyglucose (FDG-PET) in the clinically negative neck: is it likely to be superior?" Eur Arch Otorhinolaryngol. 2004. 261(9): 479-83.

4. Connell, C. A., Corry, J., Milner, A. D., et al., "Clinical impact of, and prognostic stratification by, F-18 FDG PET/CT in head and neck mucosal squamous cell carcinoma." Head Neck. 2007. 29(11): 986-95.

5. de Bondt, R. B., Nelemans, P. J., Hofman, P. A., et al., "Detection of lymph node metastases in head and neck cancer: A meta-analysis comparing US, USgFNAC, CT and MR imaging." Eur J Radiol. 2007.

6. Escott, E. J., "Positron emission tomography-computed tomography protocol considerations for head and neck cancer imaging." Semin Ultrasound CT MR. 2008. 29(4): 263-70.

7. Fleming, A. J., Jr. and Johansen, M. E., "The clinician's expectations from the use of positron emission tomography/computed tomography scanning in untreated and treated head and neck cancer patients." Curr Opin Otolaryngol Head Neck Surg. 2008. 16(2): 127-34.

8. Hollenbeak, C. S., Lowe, V. J. and Stack, B. C., Jr., "The cost-effectiveness of fluorodeoxyglucose 18-F positron emission tomography in the N0 neck." Cancer. 2001. 92(9): 2341-8.

9. Kyzas, P. A., Evangelou, E., Denaxa-Kyza, D., et al., "18F-fluorodeoxyglucose positron emission tomography to evaluate cervical node metastases in patients with head and neck squamous cell carcinoma: a meta-analysis." J Natl Cancer Inst. 2008. 100(10): 712-20.

10. Nahmias, C., Carlson, E. R., Duncan, L. D., et al., "Positron emission tomography/computerized tomography (PET/CT) scanning for preoperative staging of patients with oral/head and neck cancer." J Oral Maxillofac Surg. 2007. 65(12): 2524-35.

11. Ng, S. H., Yen, T. C., Liao, C. T., et al., "18F-FDG PET and CT/MRI in oral cavity squamous cell carcinoma: a prospective study of 124 patients with histologic correlation." J Nucl Med. 2005. 46(7): 1136-43.

12. Pentenero, M., Cistaro, A., Brusa, M., et al., "Accuracy of 18F-FDG-PET/CT for staging of oral squamous cell carcinoma." Head Neck. 2008. 30(11): 1488-96.

13. Quon, A., Fischbein, N. J., McDougall, I. R., et al., "Clinical role of 18F-FDG PET/CT in the management of squamous cell carcinoma of the head and neck and thyroid carcinoma." J Nucl Med. 2007. 48 Suppl 1: 58S-67S.

14. Roh, J. L., Yeo, N. K., Kim, J. S., et al., "Utility of 2-[18F] fluoro-2-deoxy-D-glucose positron emission tomography and positron emission tomography/computed tomography imaging in the preoperative staging of head and neck squamous cell carcinoma." Oral Oncol. 2007. 43(9): 887-93.

15. Schoder, H., Carlson, D. L., Kraus, D. H., et al., "18F-FDG PET/CT for detecting nodal metastases in patients with oral cancer staged N0 by clinical examination and CT/MRI." J Nucl Med. 2006. 47(5): 755-62.

16. Straetmans, J. M., Olthof, N., Mooren, J. J., et al., "Human papillomavirus reduces the prognostic value of nodal involvement in tonsillar squamous cell carcinomas." Laryngoscope. 2009. 119(10): 1951-7. 
17. van den Brekel, M. W., Stel, H. V., Castelijns, J. A., et al., "Cervical lymph node metastasis: assessment of radiologic criteria." Radiology. 1990. 177(2): 379-84.

18. Veit-Haibach, P., Luczak, C., Wanke, I., et al., "TNM staging with FDG-PET/CT in patients with primary head and neck cancer." Eur J Nucl Med Mol Imaging. 2007. 34(12): 1953-62.

19. Weiss, M. H., Harrison, L. B. and Isaacs, R. S., "Use of decision analysis in planning a management strategy for the stage N0 neck." Arch Otolaryngol Head Neck Surg. 1994. 120(7): 699-702.

20. Wensing, B. M., Vogel, W. V., Marres, H. A., et al., "FDG-PET in the clinically negative neck in oral squamous cell carcinoma." Laryngoscope. 2006. 116(5): 809-13. 


\title{
CHAPTER 2
}

\section{Lymph node imaging in the head and neck: overview of the present status of modern imaging techniques}

\author{
M.J. Lahaye \\ R.B.J. de Bondt \\ S.M.E. Engelen \\ G.L. Beets \\ R.G.H. Beets-Tan
}

Adapted from

Lymph node Imaging: Present Status and Future Developments, in: Clinical Blood Pool MR Imaging, p. 181-191 (Springer-Verlag) 


\section{Introduction}

This chapter will describe the present status of modern imaging techniques for identifying lymph node metastases in patients with head and neck cancer and describe the upcoming role and evidence so far of promising modern imaging techniques and new contrast agents that could help radiologists to improve their performance by non-invasive modern imaging techniques.

\section{Lymph node anatomy and physiology}

Knowledge of the anatomy and physiology of the lymphatic system and lymph nodes is important in order to have a basic understanding of lymph node imaging. Lymph nodes are small bean-shaped structures that are usually less than $2.5 \mathrm{~cm}$ in long axial diameter. They are largely distributed in drainage areas of body sites most exposed to the external environment like pharynx axis, gastrointestinal tract, lungs and limbs. Therefore the lymphatic system is an essential first line of defense against pathogens.

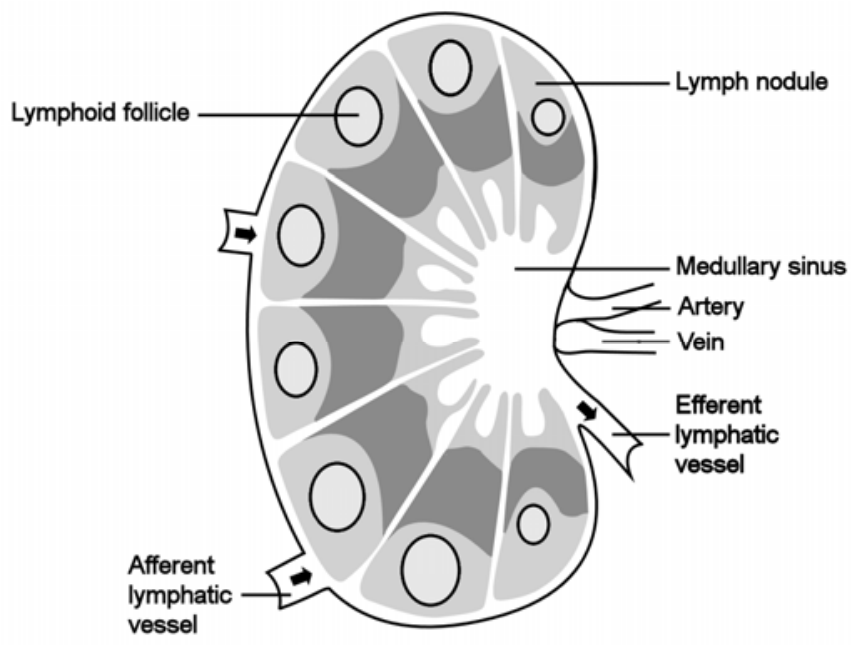

Figure 1. Schematic drawing of the anatomy of a normal lymph node.

The normal lymph node is separated into compartments, called lymph nodules and surrounded by a connective tissue capsule (figure 1). In these lymph nodules, dense masses of macrophages and lymphocytes are situated and separated by spaces called lymph sinuses. Through these lymph sinuses the lymph fluid passes on their way from the numerous afferent lymphatic vessels towards an efferent lymphatic vessel. 
The efferent vessel leaves the node at a concave area called the hilum. The lymph fluid consists chyle, proteins, fat and white blood cells of which predominantly lymphocytes. The lymphatic vessels and the lymph node pattern anatomically define the lymph fluid flow to lymph nodes. Metastasis can follow this structured lymph flow to spread to the next lymph node. Cabanas et al. demonstrated the existence of specific lymph node centers, the so-called sentinel lymph nodes (SLN), which were the primary site of metastases from penile cancer [2]. When biopsy of a SLN is negative for metastatic disease, the other lymph nodes downstream are likely to be also benign. Thus, a specific node can be the doorway for cancer to spread to other regional nodes. It is possible for a metastasis in a sentinel node to grow and block the lymphatic flow, thus redirecting lymph and tumor cells to other, possibly not ordinarily sequential, nodes. This hypothesis of a sentinel node has also been established in malignancies in the head and neck $[5 ; 6 ; 3]$

\section{Ultrasound}

During the past decades radiologists have gained a great deal of experience in lymph node imaging by means of ultrasound [5]. Several morphological characteristics of lymph nodes are assessed to distinguish malignant from benign lymph nodes. Those characteristics suggesting tumoral lymph node involvement are enlargement, round shape, irregular border, loss of the central hilum and hypo echogeneity [3]. However, none of these morphological characteristics are accurate enough in malignant node identification and therefore US only, without fine needle aspiration cytology (FNAC), cannot be relied on for clinical decision making in head and neck cancer staging. Lymph node staging in head and neck cancer, with the introduction of ultrasound guided fine needle aspiration cytology (USg-FNAC), sensitivity and specificity ranging from $63-97 \%$ and $69-100 \%$ respectively have been obtained $[1 ; 5 ; 9]$. The associated diagnostic odds ratio (DOR) for US alone was 40 whereas the DOR for USg-FNAC was significantly higher up to 260, which means an enormous improvement of the performance of preoperative cervical lymph node staging. A drawback of ultrasound in general is that the performance depends on the experience of the sonographer [2]. A further drawback of USg-FNAC in head and neck cancer staging is the poor accessibility of cervical nodes deeply located in anatomic regions such as the retropharyngeal area; for the prediction of these lymph nodes other modalities such as the crosssectional imaging modalities CT and MRI are nowadays recommended.

Therefore the recommendations in the guidelines for diagnostic work up of head and neck cancer is to primarily stage with cross sectional imaging techniques such as CT and MRI, and limit staging of head and neck cancer patients by means of US to experienced centers only. 
Above mentioned downsides on operator dependency of the USg-FNAC performance, on restricted general availability of the USg-FNAC, on its incapability to accurately access some anatomical cervical regions at risk and on its inherent invasive nature, makes USg-FNAC a less probable practical staging tool that will gain general acceptance in head and neck cancer diagnostic work up.

New ultrasound techniques such as Doppler-ultrasound combined with microbubble contrast agents or 3D US are being studied with promising results $[2 ; 4 ; 3]$

\section{Computed Tomography}

Computed Tomography (CT) has for long time been adopted as an imaging tool to identify malignant lymph nodes in several cancers. In contrast to US, CT has a much larger field of view and is less reader dependent. However, due to its low contrast resolution, conventional computed tomography techniques have primarily relied on size criteria, and size on its own is insufficient for a reliable distinction between malignant and benign lymph nodes. Prediction of cervical lymph node metastases in head and neck cancer by means of conventional single and 4-16 slice CT techniques remains low in specificity. The main criterion for the assessment of cervical lymph node involvement using CT is the maximum short axial diameter and several studies have been undertaken to determine the optimal cut off size of the maximum short axial diameter for discrimination between metastatic and nonmetastatic lymph nodes. In general, $10 \mathrm{~mm}$ is a commonly used size cut-off but a range varying from 9 to $15 \mathrm{~mm}$ has been described [4 $\mathrm{Pt} 1 ; 1]$. Other criteria, such as the presence of necrosis or extra nodal tumor spread, are used but appear to be less valuable [4; 1]. Necrosis is mainly seen in lymph nodes exceeding $10 \mathrm{~mm}$ and those lymph nodes will at first instance already be classified as malignant based on the size criterion. In the neck, with its dense and packed structures like vessels and muscles, it is very difficult with the conventional CT techniques to achieve an acceptable soft tissue contrast resolution. Advancement in multi detector CT (MDCT) techniques with 64 slice CT being introduced in patient clinics, could theoretically mean an upgrading of CT for nodal disease detection, but so far no studies exist on the 64 slice CT for evaluating head and neck cancer.

\section{FDG-PET and 18FDG-PET-CT}

18Fluorodeoxyglucose (FDG) PET has proven its additional value for the search of distant metastases in a wide variety of tumor types. Cancer cells have an increased glycolytic rate compared to normal cells, which 18 FDG-PET is able to detect. The limitation of the nowadays-available human PET machine is its low resolution and 
high irradiation dose, and consequently its inaccuracy for the detection of low-bulk tumor, requiring a size of at least $1 \mathrm{~cm} 3$ tumour volume before PET can depict it. In patients presenting with a head and neck tumor, it is of utmost importance to accurately predict lymph node metastases in the lower cervical levels (level IV and VI), because lymph node involvement in these levels show a significant higher prevalence of distant metastases, mainly to the lungs. In these patients a chest CT has so far been recommended in the work up as a first line staging tool [5].

A recent study in patients presenting with cervical lymph node metastases, in the work up to search for the unknown primary tumor and distant metastases, a 18Fluorodeoxyglucose Positron Emission Tomography (FDG-PET) scan shows to be a superior diagnostic modality above the conventional CT staging and is now recommended in these group of patients [8].

But for the loco regional staging in primary head and neck cancer only few reports are available on the use of FDG-PET. The low resolution obtained with presently available PET machines may be the reason why interpretation of PET images in the complex anatomy of the head and neck remain very difficult both for staging of the clinically $\mathrm{N}+$ neck as well as for staging of the N0 neck [ $4 \mathrm{Pt} 1$ ]. However, Myers et al. reported a trend in increased accuracy for FDG-PET compared to CT for the detection of cervical metastases in the N0 neck in HNSCC [2]. This study was performed in only 14 patients with very small series of HNSCC in the oral cavity, oropharynx and hypopharynx. Therefore, further and larger studies are needed to validate these results, also for other tumor sites in the head and neck.

The introduction of hybrid techniques for whole body staging such as the integrated PET-CT, combining anatomical and functional information leads to superior results compared to PET or CT only as a single staging tool. [3; $1 ; 8$; 12]New generation PET-CT machines improve the detection of smaller lymph node metastases and might in future concur with other radiological imaging modalities $[3 ; 1 ; 8 ; 12][7 ; 11 ; 12 ; 9 ; 12 ; 4 ; 2 ; 10 ; 11]$

\section{Magnetic Resonance Imaging (MRI)}

Conventional MR techniques with 0.5 to 1.5 Tesla powerfield systems are in general not accurate for the detection of nodal metastases in cancer patients, despite the superior contrast resolution of MR imaging as compared with all other existing conventional imaging techniques. Staging by means of MRI in head and neck cancer remains of limited use for malignant lymph node detection as shown by the recent meta-analysis in which MRI was the least performing noninvasive diagnostic modality. This is probably due to the primarily use of the short axial 
diameter as a criterion to determine a lymph node benign or malignant. In addition to the size criterion, other criteria like morphological aspects, such as necrosis or pattern of enhancement, are used in daily practice, but the diagnostic performances still have to be established in future studies.
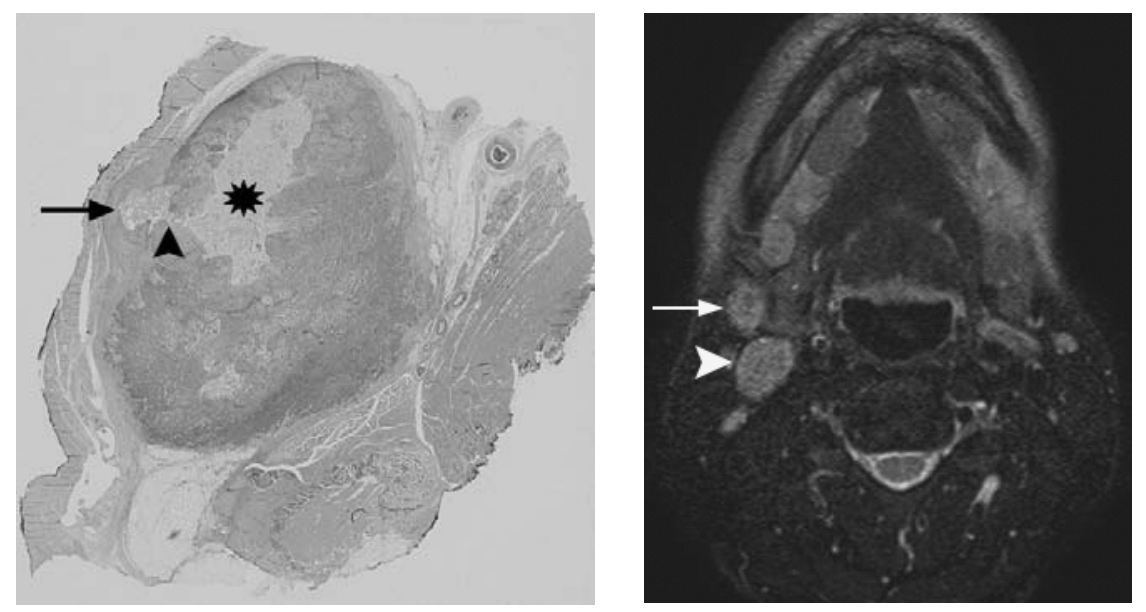

Figure 2. (A) SPIR TSE T2 weighted image in the axial plane. On the right side of the neck in level II an enlarged lymph node (arrowhead) with a short axial diameter of $12 \mathrm{~mm}$ will be classified as malignant based on the size criterion, however, cytology revealed no metastasis. Another smaller lymph node (arrow) with a short axial diameter of only $8 \mathrm{~mm}$ shows an indistinct border and a heterogeneous signal intensity. Cytology confirmed the diagnosis of a metastasis. (B) At histological examination (HE-stained) of this latter lymph node, the region of heterogeneous signal intensity corresponds to a metastasis (asterix) with extra nodal spread (arrow). Note the disruption of the lymph node capsule (arrowhead).

\section{MRI with lymph node specific contrast agents}

During the last decade, a new MR contrast agent Ultrasmall Super Paramagnetic Iron Oxide (USPIO) has been developed and in preclinical as well as clinical studies has proven to be promising for oncology patients $[2 ; 6 ; 3]$. USPIO is at present the only contrast agent that has shown the largest evidence that it is effective in nodal staging of cancer patients. Despite these results it has to be mentioned that USPIO is not approved and therefor not availible in several European countries due to the high cost-effectiveness.

USPIO is a contrast agent that undergoes phagocytosis by the reticuloendothelial system in the liver but also by macrophages located in benign lymph nodes. Uptake of USPIO within the node results in a decrease of signal intensity on $\mathrm{T}^{*}$-weighted images due to increased susceptibility artifacts. This means that benign regions in a 
node appear black on T2*-weighted images(figure 3A). In malignant nodes tumor deposits displace the macrophages. In these regions in the node no uptake of USPIO will occur (figure 3B). These regions with no uptake will be depicted as regions within the node with high signal intensity (white region). Weissleder et al. were the first to distinguish malignant from benign nodes in an animal model using USPIO [2], followed by Anzai et al. who demonstrated in a study with healthy volunteers that the uptake of USPIO reached its peak after 24 hours [1]. A recent meta-analysis of 19 USPIO MR studies showed that USPIO MRI has a sensitivity and specificity of $88 \%$ and $96 \%$, respectively for the detection of malignant nodes in various pelvic and head and neck cancers [1].

\section{MRI with Gadolinium based lymph node specific contrast agents}

Another type of MR contrast agents are the Gadolinium (Gd) based compounds, such as Gd-DTPA and MS325 (Vasovist ${ }^{\circ}$ ). These gadolinium-based contrast agents are promising for the detection of malignant lymph nodes.

Several studies have assessed the role of Gd DTPA perfusion MR imaging for the detection of malignant nodes [9]. In a series of 21 patients with squamous cell carcinomas of the head and neck, Fischbein et al. used DCE-MRI to assess cervical lymph nodes [3]. Malignant nodes in head and neck cancer displayed a significantly longer time to peak enhancement, reduced peak enhancement, decreased slope and slower wash-out, compared to normal lymph nodes.

Dynamic Contrast Enhanced (DCE) MR imaging acquires serial images following the intravenous injection of the contrast agent. Wash-in and wash-out curves can be derived from designated regions of interest (ROIs) for direct comparison, or pharmacokinetic models can be applied in order to derive permeability characteristics $[4 ; 5]$

Gd-DTPA enhanced dynamic MR imaging reflects the physiology of the microcirculation, especially the microvasculature and the extravascular space. Microcirculation in tumoral nodes generally differs from that in non-tumoral, reactive nodes. Lymphoid tissue, especially in a reactive lymph node, generally has a higher blood flow while tumour tissue in malignant nodes can be heterogeneous, slow and even retrograde. The delayed tumoral node enhancement due to a longer time to peak and lower peak enhancement is a direct reflection of the slower leakage of blood pool contrast agents from blood into the interstitial space of a tumoral node. A decreased volume of extravascular, extracellular space of metastatic tissue in lymph nodes as compared to that in reactive lymph node tissue further 
contributes to the slower leakage and wash out of contrast agents in malignant nodes.

A major drawback of the DCE-MRI is the region of interest analysis method, although embedded in the dynamic contrast-enhanced MR imaging literature. Liney et al. retrospectively evaluating three distinct methods of region of interest selection in lymph nodes in breast cancer patients [6]. Each method returned differing values, showing that further work in this area aiming at increasing reproducibility is obviously needed.

Recently Vasovist ${ }^{\circledR}$ (Gadovosfeset Trisodium, Bayer Schering Pharma, Berlin, Germany) has been introduced and approved as Gd based blood pool MR contrast agent for vascular MR imaging. Gadovosfeset Trisodium Vasovist ${ }^{\circ}$ is a formulation of a stable gadolinium diethylenetriaminepentaacetic acid (GdDTPA) chelate substituted with a diphenylcyclohexylphosphate group (gadofosveset trisodium and binds reversible to the human protein albumin.

A recent study in a rabbit model has shown that interstitial MR lymphography was feasible using Vasovist ${ }^{\oplus}$ contrast agent [6]. Malignant nodes in this rabbit model showed a longer time to peak enhancement and reduced peak enhancement than benign nodes. So far no human studies in cancer lymph node metastases have been published but need to be initiated to prove the role of this contrast agent.

\section{Conclusion}

Preoperative selection of patients for a tailored treatment can only be made when more reliable selection tools than the existing ones will become available.

Staging by clinical examination only does not reach a high level of accuracy for selecting these patients. The simultaneous advancement in imaging techniques during the past decade, providing morphological and functional information in one single examination, offers clinicians nowadays a promising tool to distinguish between the high risk, for whom a multimodality treatment could be the only curative option and the low risk patients for whom local and distant tumour control could be obtained with surgical excision only. This chapter aimed at describing the present status of modern imaging techniques for identifying nodal disease in patients with head and neck cancer. 


\section{References}

1. Anzai, Y., McLachlan, S., Morris, M., et al., "Dextran-coated superparamagnetic iron oxide, an MR contrast agent for assessing lymph nodes in the head and neck." AJNR Am J Neuroradiol. 1994. 15(1): 87-94.

2. Cabanas, R. M., "An approach for the treatment of penile carcinoma." Cancer. 1977. 39(2): 456-66.

3. Connell, C. A., Corry, J., Milner, A. D., et al., "Clinical impact of, and prognostic stratification by, F-18 FDG PET/CT in head and neck mucosal squamous cell carcinoma." Head Neck. 2007. 29(11): 986-95.

4. Curtin, H. D., Ishwaran, H., Mancuso, A. A., et al., "Comparison of CT and MR imaging in staging of neck metastases." Radiology. 1998. 207(1): 123-30.

5. de Bondt, R. B., Nelemans, P. J., Hofman, P. A., et al., "Detection of lymph node metastases in head and neck cancer: A meta-analysis comparing US, USgFNAC, CT and MR imaging." Eur J Radiol. 2007.

6. de Lussanet, Q. G., Backes, W. H., Griffioen, A. W., et al., "Dynamic contrast-enhanced magnetic resonance imaging of radiation therapy-induced microcirculation changes in rectal cancer." Int J Radiat Oncol Biol Phys. 2005. 63(5): 1309-15.

7. Escott, E. J., "Positron emission tomography-computed tomography protocol considerations for head and neck cancer imaging." Semin Ultrasound CT MR. 2008. 29(4): 263-70.

8. Fischbein, N. J., Noworolski, S. M., Henry, R. G., et al., "Assessment of metastatic cervical adenopathy using dynamic contrast-enhanced MR imaging." AJNR Am J Neuroradiol. 2003. 24(3): 301-11.

9. Fleming, A. J., Jr. and Johansen, M. E., "The clinician's expectations from the use of positron emission tomography/computed tomography scanning in untreated and treated head and neck cancer patients." Curr Opin Otolaryngol Head Neck Surg. 2008. 16(2): 127-34.

10. Herborn, C. U., Lauenstein, T. C., Vogt, F. M., et al., "Interstitial MR lymphography with MS-325: characterization of normal and tumor-invaded lymph nodes in a rabbit model." AJR Am J Roentgenol. 2002. 179(6): 1567-72.

11. Jeong, H. S., Chung, M. K., Son, Y. I., et al., "Role of 18F-FDG PET/CT in management of high-grade salivary gland malignancies." J Nucl Med. 2007. 48(8): 1237-44.

12. Knappe, M., Louw, M. and Gregor, R. T., "Ultrasonography-guided fine-needle aspiration for the assessment of cervical metastases." Arch Otolaryngol Head Neck Surg. 2000. 126(9): 10916.

13. Kvistad, K. A., Rydland, J., Smethurst, H. B., et al., "Axillary lymph node metastases in breast cancer: preoperative detection with dynamic contrast-enhanced MRI." Eur Radiol. 2000. 10(9): 1464-71.

14. Kyzas, P. A., Evangelou, E., Denaxa-Kyza, D., et al., "18F-fluorodeoxyglucose positron emission tomography to evaluate cervical node metastases in patients with head and neck squamous cell carcinoma: a meta-analysis." J Natl Cancer Inst. 2008. 100(10): 712-20.

15. Liney, G. P., Gibbs, P., Hayes, C., et al., "Dynamic contrast-enhanced MRI in the differentiation of breast tumors: user-defined versus semi-automated region-of-interest analysis." J Magn Reson Imaging. 1999. 10(6): 945-9.

16. Lurie, D. M., Seguin, B., Schneider, P. D., et al., "Contrast-assisted ultrasound for sentinel lymph node detection in spontaneously arising canine head and neck tumors." Invest Radiol. 2006. 41(4): 415-21.

17. McGuirt, W. F., Williams, D. W., 3rd, Keyes, J. W., Jr., et al., "A comparative diagnostic study of head and neck nodal metastases using positron emission tomography." Laryngoscope. 1995. 105 (4 Pt 1): 373-5. 
18. Mende, U., Krempien, R., Hassfeld, S., et al., "[3D-ultrasound: a valuable adjunct for therapy planning and follow-up of head and neck tumours]." Ultraschall Med. 2002. 23(2): 101-7.

19. Misselwitz, B., Platzek, J. and Weinmann, H. J., "Early MR lymphography with gadofluorine $\mathrm{M}$ in rabbits." Radiology. 2004. 231(3): 682-8.

20. Myers, L. L., Wax, M. K., Nabi, H., et al., "Positron emission tomography in the evaluation of the N0 neck." Laryngoscope. 1998. 108(2): 232-6.

21. Nahmias, C., Carlson, E. R., Duncan, L. D., et al., "Positron emission tomography/computerized tomography (PET/CT) scanning for preoperative staging of patients with oral/head and neck cancer." J Oral Maxillofac Surg. 2007. 65(12): 2524-35.

22. Ng, S. H., Yen, T. C., Liao, C. T., et al., "18F-FDG PET and CT/MRI in oral cavity squamous cell carcinoma: a prospective study of 124 patients with histologic correlation." J Nucl Med. 2005. 46(7): 1136-43.

23. Pentenero, M., Cistaro, A., Brusa, M., et al., "Accuracy of 18F-FDG-PET/CT for staging of oral squamous cell carcinoma." Head Neck. 2008. 30(11): 1488-96.

24. Plaat, R. E., de Bree, R., Kuik, D. J., et al., "Prognostic importance of paratracheal lymph node metastases." Laryngoscope. 2005. 115(5): 894-8.

25. Quon, A., Fischbein, N. J., McDougall, I. R., et al., "Clinical role of 18F-FDG PET/CT in the management of squamous cell carcinoma of the head and neck and thyroid carcinoma." J Nucl Med. 2007. 48 Suppl 1: 58S-67S.

26. Regelink, G., Brouwer, J., de Bree, R., et al., "Detection of unknown primary tumours and distant metastases in patients with cervical metastases: value of FDG-PET versus conventional modalities." Eur J Nucl Med Mol Imaging. 2002. 29(8): 1024-30.

27. Rijpkema, M., Kaanders, J. H., Joosten, F. B., et al., "Method for quantitative mapping of dynamic MRI contrast agent uptake in human tumors." J Magn Reson Imaging. 2001. 14(4): 457-63.

28. Roh, J. L., Yeo, N. K., Kim, J. S., et al., "Utility of 2-[18F] fluoro-2-deoxy-D-glucose positron emission tomography and positron emission tomography/computed tomography imaging in the preoperative staging of head and neck squamous cell carcinoma." Oral Oncol. 2007. 43(9): 887-93.

29. Roren, A., Mayoux-Benhamou, M. A., Fayad, F., et al., "Comparison of visual and ultrasound based techniques to measure head repositioning in healthy and neck-pain subjects." Man Ther. 2009. 14(3): 270-7.

30. Schroder, R. J., Pegios, W., Hunerbein, M., et al., "[Magnetic resonance tomography and endosonography in the preoperative staging of advanced rectal carcinomas after hyperthermoradiochemotherapy]." Rofo. 1997. 166(3): 199-205.

31. Schwartz, D. L., Ford, E., Rajendran, J., et al., "FDG-PET/CT imaging for preradiotherapy staging of head-and-neck squamous cell carcinoma." Int J Radiat Oncol Biol Phys. 2005. 61(1): 129-36.

32. Starek, I., Koranda, P. and Benes, P., "Sentinel lymph node biopsy: A new perspective in head and neck mucosal melanoma?" Melanoma Res. 2006. 16(5): 423-7.

33. Steinkamp, H. J., Zwicker, C., Langer, M., et al., "[Reactive enlargement of cervical lymph nodes and cervical lymph node metastases: sonography (M/Q quotient) and computed tomography]." Aktuelle Radiol. 1992. 2(4): 188-95.

34. Takes, R. P., Righi, P., Meeuwis, C. A., et al., "The value of ultrasound with ultrasound-guided fine-needle aspiration biopsy compared to computed tomography in the detection of regional metastases in the clinically negative neck." Int J Radiat Oncol Biol Phys. 1998. 40(5): 1027-32.

35. Tanis, P. J., Nieweg, O. E., van den Brekel, M. W., et al., "Dilemma of clinically nodenegative head and neck melanoma: outcome of "watch and wait" policy, elective lymph node dissection, and sentinel node biopsy--a systematic review." Head Neck. 2008. 30(3): 380-9. 
36. Tregnaghi, A., De Candia, A., Calderone, M., et al., "Ultrasonographic evaluation of superficial lymph node metastases in melanoma." Eur J Radiol. 1997. 24(3): 216-21.

37. van den Brekel, M. W., Castelijns, J. A., Stel, H. V., et al., "Modern imaging techniques and ultrasound-guided aspiration cytology for the assessment of neck node metastases: a prospective comparative study." Eur Arch Otorhinolaryngol. 1993. 250(1): 11-7.

38. Veit-Haibach, P., Luczak, C., Wanke, I., et al., "TNM staging with FDG-PET/CT in patients with primary head and neck cancer." Eur J Nucl Med Mol Imaging. 2007. 34(12): 1953-62.

39. Wagner, A., Kermer, C., Zettinig, G., et al., "Validity of sentinel lymph node (SLN) detection following adjuvant radiochemotherapy (RCT) in head and neck squamous cell carcinoma (HNSCC)." Technol Cancer Res Treat. 2007. 6(6): 655-60.

40. Weissleder, R., Elizondo, G., Wittenberg, J., et al., "Ultrasmall superparamagnetic iron oxide: an intravenous contrast agent for assessing lymph nodes with MR imaging." Radiology. 1990. 175(2): 494-8.

41. Will, O., Purkayastha, S., Chan, C., et al., "Diagnostic precision of nanoparticle-enhanced MRI for lymph-node metastases: a meta-analysis." Lancet Oncol. 2006. 7(1): 52-60.

42. Zenk, J., Bozzato, A., Hornung, J., et al., "Neck lymph nodes: prediction by computer-assisted contrast medium analysis?" Ultrasound Med Biol. 2007. 33(2): 246-53. 
$\longrightarrow$ 


\title{
CHAPTER 3
}

Detection of lymph node metastases in head and neck cancer: A meta-analysis comparing US, USgFNAC, $C T$ and MR imaging

\author{
R.B.J. de Bondt \\ P.J. Nelemans \\ P.A.M. Hofman \\ J.W. Casselman \\ B. Kremer \\ J.M.A. van Engelshoven \\ R.G.H. Beets-Tan
}

European Journal of Radiology 2007;64:266-272 


\section{Abstract}

Purpose: To perform a meta-analysis comparing ultrasonography (US), US guided fine needle aspiration cytology (USgFNAC), computed tomography (CT), and magnetic resonance imaging (MRI) in the detection of lymph node metastases in head and neck cancer.

Methods: MEDLINE, EMBASE and Cochrane databases were searched (January 1990-January 2006) for studies reporting diagnostic performances of US, USgFNAC, CT, and MRI to detect cervical lymph node metastases. Two reviewers screened text and reference lists of potentially eligible articles. Criteria for study inclusion: (1) histopathology was the reference standard, (2) primary tumours and metastases were squamous cell carcinoma and (3) data were available to construct $2 \times 2$ contingency tables. Meta-analysis of pairs of sensitivity and specificity was performed using bivariate analysis. Summary estimates for diagnostic performance used were sensitivity, specificity, diagnostic odds ratios (DOR) (95\% confidence intervals) and summary receiver operating characteristics (SROC) curves.

Results: From seventeen articles, 25 data sets could be retrieved. Eleven articles studied one modality: US ( $n=4)$; USgFNAC $(n=1)$; CT $(n=3)$; MRI $(n=3)$. Six articles studied two or more modalities: US and CT $(n=2)$; USgFNAC and CT $(n=1)$; CT and MRI $(n=1)$; MRI and MRI-USPIO (Sinerem) $(n=2)$; US, USgFNAC, CT and MRI $(n=1)$. USgFNAC (AUC $=0.98)$ and US $($ AUC $=$ 0.95 ) showed the highest areas under the curve (AUC). MRI-USPIO (AUC = $0.89)$ and $\mathrm{CT}(\mathrm{AUC}=0.88)$ had similar results. MRI showed an AUC $=0.79$. USgFNAC showed the highest DOR $(\mathrm{DOR}=260)$ compared to US $(\mathrm{DOR}=$ 40), MRI-USPIO (DOR = 21), CT $(\mathrm{DOR}=14)$ and MRI $(\mathrm{DOR}=7)$.

Conclusion: USgFNAC showed to be the most accurate imaging modality to detect cervical lymph node metastases. 


\section{Introduction}

Of all head and neck tumours more than $90 \%$ are squamous cell carcinomas (SCC) [1]. The presence of one isolated lymph node metastasis (stage N1) decreases the prognosis with $50 \%$, while multiple metastases decrease the prognosis even more dramatically; therefore, the lymph node status is one of the most important predictors of poor prognosis in head and neck tumours [2-4].

Management in patients presenting with lymph node metastases could consist of a neck dissection in which selected or all lymph node levels in the neck are removed. This could be followed by radiation therapy depending on histological findings like extra nodal spread or presence of multiple metastases. It has to be kept in mind that a neck dissection as well as radiation therapy have a considerable morbidity and mortality [5-7]. Therefore, accurate assessment of the lymph node status is important for the choice of treatment.

Radiological imaging modalities, like computed tomography (CT) and magnetic resonance imaging (MRI), can be used to support treatment decisions when an unexpected lymph node metastasis is detected on the opposite side in the neck or when it is detected on the ipsilateral side where it is not suspected. Alternative diagnostic modalities are ultrasonography (US) and ultrasonography guided fine needle aspiration cytology (USgFNAC). However, because of the high operator dependency of these latter modalities it generally remains restricted to expert referral centers. An important drawback of US and USgFNAC is the inability to detect (high) retropharyngeal lymph nodes. Important questions, whether these imaging techniques perform well enough and whether one modality is superior to others, need answers.

Regarding the literature, diagnostic performances of studies are difficult to compare due to a large variety in study designs, study populations, imaging modalities and techniques. Also, only few reports compared all radiological imaging modalities in one study. A meta-analysis of the literature is a tool for these problems as it provides in summarizing the results of individual radiological diagnostic studies.

Such a meta-analysis could provide answers to two relevant questions in the management of patients with a head and neck tumour. Firstly, what is the relative performance of these modalities and secondly, are these modalities accurate enough to further reduce the potential risk of occult lymph node metastases? 
The aim of this study was to perform a meta-analysis of radiological imaging modalities for the detection of lymph node metastases in patients with SCC of the head and neck; US, USgFNAC, CT, and MR imaging were compared.

\section{Materials and methods}

\section{Literature research}

A computerized systematic literature search was performed by one reader. Abstracts were selected of articles reporting the diagnostic performance of ultrasound (US), US guided fine needle aspiration cytology (USgFNAC), computed tomography (CT), and magnetic resonance imaging (MRI) in the detection of lymph node metastases in head and neck tumours. The MEDLINE database from January 1990 to January 2006 was searched with the following search terms as keywords: (a) "head and neck neoplasm" (medical subject heading, or $\mathrm{MeSH}$ ), (b) lymph nodes $(\mathrm{MeSH})$ or "lymphatic system $(\mathrm{MeSH})$ " or "neoplasm metastases" (MeSH) and (c) "diagnostic imaging" (MeSH). The EMBASE and Cochrane databases were searched by using(a) "head and neck",(b) "neoplasm",(c) "lymph nodes" or "lymphatic system", (d) "metastases" and (e) "imaging” as text words.

From the selected abstracts, two reviewers separately screened the full text of these potentially eligible articles in which US, USgFNAC, CT, and/or MR imaging were evaluated. Reference lists were manually screened for additional relevant articles.

\section{Inclusion criteria}

Based on the full text, studies were selected if they fullfilled all of the following inclusion criteria: (a) histopathology findings (specimens obtained at surgery or at lymph node biopsy) were used as the reference standard, (b) the primary tumour and lymph node metastases were squamous cell carcinomas and (c) sufficient data were presented to construct a $2 \times 2$ contingency table (sensitivity and/or specificity with absolute numbers of false positive (FP), false negative (FN), true positive (TP) and true negative (TN) findings) for the imaging modalities compared with the reference standard.

\section{Exclusion criteria}

Studies were excluded if raw data, such as two-by-two tables for statistical analysis, were not presented. Reviews, letters to the author, comments and case reports were excluded. 


\section{Data extraction}

Two reviewers separately extracted relevant data from each article by using a standard data form. Neither reviewer was blinded to information regarding authors, journal name, or year of publication. In case of disagreement, a third reviewer would assess these articles.

Characteristics of the selected articles were noted: (a) reporting of the study population and number of patients, (b) reporting of the diagnostic test(s) and reference test, (c) reporting of criteria for positive lymph node involvement; the size (expressed in axial, longitudinal or ratio of both) with cut-off points, presence of necrosis, appearance of extra nodal spread and enhancement on CT or MR imaging and (d) year of publication and author.

\section{Statistical analysis}

Results on absolute numbers of false positive (FP), false negative (FN), true positive (TP) and true negative (TN) findings for the imaging modalities were analyzed using the bivariate model [8]. Bivariate analysis is a method for metaanalyzing diagnostic studies. The method has two advantages. Firstly, it accounts for the trade-off between sensitivity and specificity in diagnostic studies, which results from between-study variation in test threshold, and secondly, it incorporates between-study heterogeneity that is to be expected in diagnostic studies. Pairs of sensitivity and specificity, which are derived from the individual studies under review, are jointly analyzed, incorporating any correlation that might exist between these two measures using a random effects model [8].

Summary estimates for diagnostic performance that were estimated were sensitivity, specificity, diagnostic odds ratios (DOR) with $95 \%$ confidence intervals and summary receiver operating characteristics (SROC) curves. The diagnostic odds ratio (DOR) is a measure for diagnostic performance of a test, which combines sensitivity and specificity into one measure. A DOR of 1 implies that the test has no discriminatory power at all; the larger the DOR, the better the test discriminates between patients with and without the target disorder (in this metaanalysis, the presence of metastases). The estimates of DOR, which were derived from the bivariate model, were used to fit SROC curves through the observed pairs of sensitivity and specificity. In this way, it can be visualized that a point $\mathrm{P} 1$, which represents a study with observed false positive rate (FPR) of $20 \%$ and observed true positive rate (TPR) of $75 \%$, and a point $\mathrm{P} 2$, which represents another study with observed FPR of $8 \%$ and observed TPR of $50 \%$, are roughly concordant with one SROC curve and represent similar diagnostic performance [9]. In order not to 
extend beyond the empirical range of data, SROC curves were restricted within the range of FPR values that were actually observed in the included studies (partial SROC curves). For all analyses we used SPSS 13.0 for Windows.

\section{Results}

The computerized search revealed a total of 831 articles. After reading the abstracts, 88 articles were found to be relevant, of which 17 studies fullfilled the criteria for inclusion after reading the full text [10-26]. There was no disagreement between the two readers in selecting articles. Reasons for exclusion of studies were as follows: (a) not sufficient or inconclusive information was presented on the histopathology findings as the reference standard $(n=26)$, (b) not sufficient or inconclusive data were presented to construct a $2 \times 2$ contingency table (sensitivity and/or specificity with absolute numbers of false positive (FP), false negative (FN), true positive (TP) and true negative (TN) findings) ( $n=16$ ), (c) study data were reported elsewhere in more detail $(n=2)$ and (d) studies focused on another subject; search for the unknown primary tumour $(n=4)$, overview of imaging techniques $(n=12)$, search for distant metastases $(n=2)$, search for extra nodal spread $(n=2)$, other subjects such as genetics, survival study and vascular studies $(n=6)$. From the 17 articles, 25 data sets were retrieved. We identified 7, 3, 8, 5, 2 studies for US, USgFNAC, CT, MRI and MRI-USPIO (Sinerem), respectively. Eleven articles studied one diagnostic modalities; US $(n=4)$, USgFNAC $(n=1)$, CT $(n=3)$ and MRI $(n=3)$. Six articles compared two or more diagnostic modality; US and CT $(n=2)$, USgFNAC and CT $(n=1)$, CT and MRI $(n=1)$, MRI and MRI-USPIO $(n=2)$, US and USgFNAC and CT and MRI $(n=1)$.

Figure 1 shows partial SROC curves for all modalities. This figure visualizes the trade off between sensitivity and specificity. It illustrates that with USgFNAC the sensitivity of $80 \%$ can be reached without much loss of specificity, whereas with CT the sensitivity of $80 \%$ is only reached at the expanse of more false positive findings.

Table 1 presents a summary of study and patient characteristics. A large variation in reported sensitivity and specificity was observed for all imaging modalities. For US for example, sensitivity values range from 63\% to 97\%. Generally, low sensitivity values are associated with high specificity and vice versa, but there are exceptions, such as the study by van den Brekel et al. [26] (both sensitivity and specificity are 75\%) and the study by Steinkamp et al. [15] with a sensitivity of 
$93 \%$ and a specificity of $95 \%$. The prevalence of positive lymph nodes in the reviewed studies also show much variation: they range between $20 \%$ and $77 \%$.

Table 2 summarizes the size and morphologic criteria for determination of lymph node metastases per author and imaging modality. Those criteria vary not only between the different modalities but also within studies on US, USgFNAC, CT and MRI.

Table 3 presents summary estimates for diagnostic performance for each modality: sensitivity, specificity and DOR with $95 \%$ confidence intervals. The highest DOR was found for USgFNAC (DOR $=260)$. This was significantly higher $(p<0.05)$ than that for US (DOR $=40)$, MRI-USPIO (Sinerem) $(\mathrm{DOR}=21)$, CT $(\mathrm{DOR}=$ 14 ) and MRI (DOR = 7). Other pair wise comparisons of the modalities showed no significant differences in diagnostic performance between modalities except for the comparison between US and MRI in which US performs significantly better than MRI ( $p=0.006$ ). Mean sensitivity of $87 \%$ was highest for US (with $95 \%$ CI 76-93\%), and specificity of 98\% was highest for USgFNAC (95\% CI 93-99.7\%).

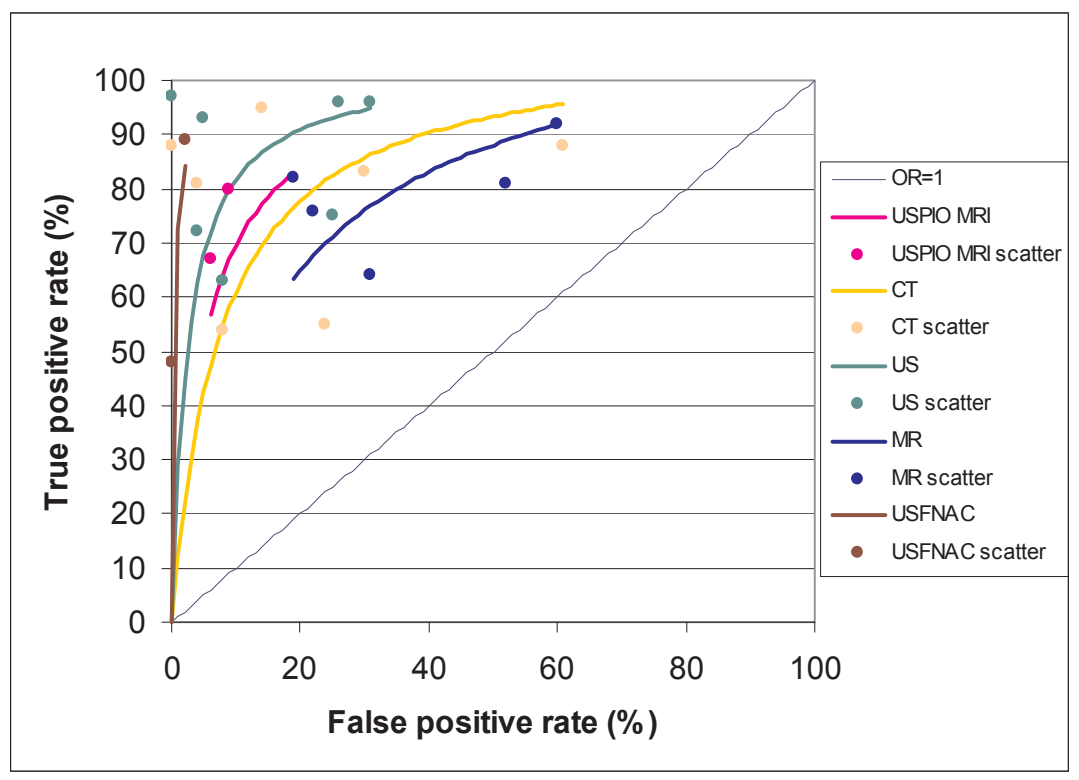

Figure 1. Partial Summary ROC curves comparing the accuracy of ultrasound guided fine needle aspiration cytology (USgFNAC), ultrasound (US), computed tomography (CT) and magnetic resonance imaging (MRI) without and with the use of ultra-small particles iron oxide (USPIO) (MRI-USPIO) in the detection of cervical lymph node metastases in head and neck tumour. The scatter of observed pairs of sensitivity and specificity are presented around the fitted SROC curves. OR: odds ratio. 
Table 1. Study characteristics and included data sets for each imaging modality, ultrasound (US), ultrasound guided fine needle aspiration cytology (USgFNAC), computed tomography (CT) and magnetic resonance imaging (MRI) without and with the use of USPIOa (MRI-USPIO), of the included articles

\begin{tabular}{|c|c|c|c|c|c|c|c|c|c|}
\hline $\begin{array}{l}\text { Imaging } \\
\text { modality }\end{array}$ & $\begin{array}{c}\mathrm{Nr} \text { of } \\
\text { patients }\end{array}$ & $\begin{array}{c}\text { Sensitivity } \\
\text { (\%) }\end{array}$ & $\begin{array}{l}\text { Specificity } \\
(\%)\end{array}$ & $\mathrm{TP}^{\mathrm{b}}$ & $\mathrm{FN}^{\mathrm{c}}$ & $\mathrm{TN}^{\mathrm{d}}$ & $\mathrm{FP}^{\mathrm{e}}$ & $\begin{array}{l}\text { Prevalen } \\
\text { lymph } \mathrm{r} \\
\text { metastase }\end{array}$ & References \\
\hline US & 48 & 72 & 96 & 18 & 4 & 24 & 2 & 46 & Haberal et al. [10] \\
\hline US & 184 & 63 & 92 & 91 & 54 & 95 & 8 & 58 & v. d. Brekel et al. [11] \\
\hline US & 25 & 97 & 100 & 35 & 1 & 34 & 0 & 51 & Takeuchi et al. [12] \\
\hline US & 38 & 96 & 69 & 50 & 2 & 69 & 31 & 34 & Danninger et al. [13] \\
\hline US & 132 & 75 & 75 & 82 & 27 & 53 & 18 & 61 & v. d. Brekel et al. [26] \\
\hline US & 123 & 96 & 74 & 74 & 3 & 34 & 12 & 62 & Steinkamp et al. [14] \\
\hline US & 138 & 93 & 95 & 79 & 6 & 50 & 3 & 62 & Steinkamp et al. [15] \\
\hline USgFNAC & 56 & 89 & 98 & 66 & 8 & 105 & 2 & 41 & Knappe et al. [16] \\
\hline USgFNAC & 50 & 48 & 100 & 12 & 13 & 36 & 0 & 41 & Takes et al. [17] \\
\hline USgFNAC & 67 & 90 & 100 & 56 & 6 & 29 & 0 & 68 & v. d. Brekel et al. [26] \\
\hline $\mathrm{CT}$ & 48 & 81 & 96 & 18 & 4 & 24 & 2 & 46 & Haberal et al. [10] \\
\hline $\mathrm{CT}$ & 196 & 88 & 39 & 69 & 9 & 46 & 72 & 40 & Curtin et al. [18] \\
\hline CT & 41 & 95 & 86 & 18 & 1 & 19 & 3 & 46 & McGuirt et al. [19] \\
\hline CT & 28 & 88 & 100 & 21 & 3 & 7 & 0 & 77 & Carvalho et al. [20] \\
\hline CT & 48 & 55 & 76 & 15 & 12 & 16 & 5 & 56 & Freire et al. [21] \\
\hline CT & 50 & 54 & 92 & 14 & 12 & 35 & 3 & 41 & Takes et al. [17] \\
\hline CT & 132 & 83 & 70 & 90 & 18 & 49 & 21 & 61 & v. d. Brekel et al. [26] \\
\hline CT & 138 & 93 & 66 & 79 & 6 & 35 & 18 & 62 & Steinkamp et al. [15] \\
\hline MRI & 196 & 81 & 48 & 63 & 15 & 57 & 61 & 40 & Curtin et al. [18] \\
\hline MRI & 12 & 64 & 69 & 7 & 4 & 9 & 4 & 46 & Braams et al. [22] \\
\hline MRI & 132 & 82 & 81 & 86 & 19 & 56 & 13 & 60 & v. d. Brekel et al. [26] \\
\hline MRI & 27 & 76 & 78 & 16 & 5 & 7 & 2 & 70 & Feinmesser et al. [24] \\
\hline MRI & 81 & 92 & 40 & 93 & 8 & 166 & 250 & 20 & Sigal et al. [25] \\
\hline MRI-USPIO & 81 & 80 & 81 & 81 & 20 & 337 & 79 & 20 & Sigal et al. [25] \\
\hline MRI-USPIO & 11 & 67 & 94 & 28 & 14 & 46 & 3 & 46 & Anzai et al. [23] \\
\hline
\end{tabular}

a USPIO: ultra-small particles iron oxide. b TP: true positive.

c FN: false negative. d TN: true negative. e FP: false positive. 
Table 2. Size and morphologic criteria for determination of cervical lymph node metastases as reported by different authors

\begin{tabular}{|c|c|c|c|}
\hline Author & Modality & Size criteria & Morphologic criteria \\
\hline \multirow[t]{3}{*}{ Haberal et al. [10] } & \multirow[t]{2}{*}{ US } & $>10 \mathrm{~mm}$ longest diameter & Contour irregularity \\
\hline & & Long/short diameter $<2$ & Heterogenous pattern \\
\hline & CT & $10-15 \mathrm{~mm}$ diameter & $\begin{array}{l}\text { Contour irregularity, heterogeneity, } \\
\text { necrosis rim enhancement }\end{array}$ \\
\hline van den Brekel et al. [11] & US & $7 \mathrm{~mm}$ (level II) and $6 \mathrm{~mm}$ & - \\
\hline Takeuchi et al. [12] & US & $\begin{array}{l}\text { Short/long diameter (no cut off } \\
\text { point) }\end{array}$ & Internal irregularity \\
\hline Danninger et al. [13] & US & Not specified & Not specified \\
\hline \multirow[t]{4}{*}{ van den Brekel et al. [26] } & US & $\mathrm{N} 07 \mathrm{~mm}, \mathrm{~N}+9 \mathrm{~mm}$ & - \\
\hline & USgFNAC & N0 3-4 mm, N+13-14 mm & - \\
\hline & $\mathrm{CT}$ & $>11 \mathrm{~mm}$ (level II) and >10 mm & Necrosis \\
\hline & MRI & $>11 \mathrm{~mm}$ (level II) and >10 mm & Necrosis \\
\hline Steinkamp et al. [14] & US & $>10 \mathrm{~mm}$ longest diameter & Ovoid form, hypo echogenicity \\
\hline \multirow[t]{2}{*}{ Steinkamp et al. [15] } & US & Long/short ratio $<2$ & - \\
\hline & CT & $>10 \mathrm{~mm}$ maximal diameter & Irregularity/hypo density \\
\hline Knappe et al. [16] & USgFNAC & $>8-10 \mathrm{~mm}$, long/short ratio $<2$ & $\begin{array}{l}\text { Round form, narrow/absent hilum, } \\
\text { central lucency, and irregular } \\
\text { capsule }\end{array}$ \\
\hline \multirow[t]{3}{*}{ Takes et al. [17] } & \multirow[t]{2}{*}{ USgFNAC } & $>5 \mathrm{~mm}$ short diameter & - \\
\hline & & $>7 \mathrm{~mm}$ short diameter & $\begin{array}{l}\text { Round > ovoid shape, centrally } \\
\text { hypo echogene, absence of hilum }\end{array}$ \\
\hline & $\mathrm{CT}$ & $>10 \mathrm{~mm}$ & Round, necrosis, rim enhancement \\
\hline \multirow[t]{2}{*}{ Curtin et al. [18] } & $\mathrm{CT}$ & $10-15 \mathrm{~mm}$ longest diameter & Internal abnormalities \\
\hline & MRI & $10-15 \mathrm{~mm}$ longest diameter & Internal abnormalities \\
\hline McGuirt et al. [19] & CT & $>15 \mathrm{~mm}$ level II, $10 \mathrm{~mm}$ elsewhere & Necrosis, contour irregularity \\
\hline Carvalho et al. [20] & $\mathrm{CT}$ & $>15 \mathrm{~mm}$ longest diameter & Rim enhancement \\
\hline Freire et al. [21] & CT & $15 \mathrm{~mm}$ level II, $10 \mathrm{~mm}$ elsewhere & Central necrosis \\
\hline \multirow[t]{3}{*}{ Braams et al. [22] } & \multirow[t]{3}{*}{ MRI } & $\begin{array}{l}>11 \mathrm{~mm} \text { short diameter level II, } \\
10 \mathrm{~mm} \text { elsewhere }\end{array}$ & Irregular or rim enhancement \\
\hline & & $\begin{array}{l}\text { Clusters of }>3 \text { nodes } 9-11 \mathrm{~mm} \text { level } \\
\text { II }\end{array}$ & \\
\hline & & $\begin{array}{l}\text { Clusters of }>3 \text { nodes } 8-9 \mathrm{~mm} \\
\text { elsewhere }\end{array}$ & \\
\hline Anzai et al. [23] & MRI-USPIO & Minimal size on T1 WI? & Irregularity on T2 WI \\
\hline Feinmesser et al. [24] & MRI & $>15 \mathrm{~mm}$ longest diameter & Necrosis on T2 WI \\
\hline \multirow[t]{2}{*}{ Sigal et al. [25] } & MRI & $>10 \mathrm{~mm}$ short diameter on $\mathrm{T} 1 \mathrm{WI}$ & \\
\hline & \multicolumn{2}{|c|}{ MRI-USPIO - } & Hyper intensity on $\mathrm{T}^{*}$ \\
\hline
\end{tabular}


Table 3. Summary estimates for mean sensitivity, mean specificity and diagnostic odds ratio (DOR) from the bivariate model, with corresponding $95 \%$ confidence intervals $(95 \% \mathrm{CI})$

\begin{tabular}{lccc}
\hline Imaging modality & Mean sensitivity 95\% CI & Mean specificity 95\% CI & Mean DOR 95\% CI \\
\hline CT & $0.81(0.68-0.90)$ & $0.76(0.62-0.87)$ & $14(6.6-29.7)$ \\
US & $0.87(0.76-0.93)$ & $0.86(0.74-0.93)$ & $40(17.8-90.1)$ \\
MRI & $0.81(0.65-0.91)$ & $0.63(0.43-0.80)$ & $7(3.1-17.8)$ \\
USgFNAC & $0.80(0.57-0.92)$ & $0.98(0.93-0.997)$ & $260(51.7-1306)$ \\
USPIO-MRI & $0.74(0.44-0.91)$ & $0.88(0.66-0.96)$ & $21(5.5-78.1)$ \\
\hline
\end{tabular}




\section{Discussion}

The current meta-analysis shows that ultrasound guided fine needle aspiration cytology (USgFNAC) has the best diagnostic performance for the detection of cervical lymph node metastases. Ultrasound alone is also associated with high DOR, whereas computed tomography (CT) and magnetic resonance imaging (MRI) perform less well.

The findings of this study are contrary to daily practice where CT and MRI are more commonly used to detect cervical lymph node metastases. The main advantage of CT and MRI are the lower inter-observer variation, and these techniques are, in general, less time consuming compared to USgFNAC and US. Furthermore, CT and MRI are relatively standardized techniques that can be performed at most institutions and can be interpreted by general radiologists with no specific expertise in head and neck imaging. This is in contrast to US and USgFNAC which are not popular tools in the work up of patients in general hospitals, and are generally only performed by experienced radiologists in referral hospitals.

The question whether imaging alone is accurate enough to guide treatment decisions in patients with head and neck malignancies depends on the ability of imaging techniques to rule out the presence of occult metastases. It has been proposed that a more conservative approach regarding management of the clinically negative neck could be allowed, if the risk of occult lymph node metastases could be reduced to below 20\% [27]. This would mean that the probability of metastases given a negative result of imaging (post-test probability) should drop below $20 \%$ and that high sensitivity of imaging is a minimal condition that should be satisfied. However, specificity must also be acceptable, because the power to rule out a diagnosis depends both on sensitivity and specificity [28]. Only two studies on US [12] and [15], one study on CT [19] and none of the studies on MRI showed high sensitivity (>90\%) combined with acceptable specificity. There is one study by Nieuwenhuis et al., which is not included in this review that reports that USgFNAC enables to decrease the risk of occult metastases to almost 20\% [29]. In the literature only three studies could be found that explicitly addressed the evaluation of clinically N0 necks [17], [26] and [30]. Two of these studies are included in this review [17] and [26].

There are two methodological issues concerning this meta-analysis that need to be addressed. Figure 1 shows that there is scatter of observed pairs of sensitivity and specificity around the fitted SROC curves. This scatter implies between-study 
variability that is not explained by between-study variations in test threshold. Such heterogeneity of study results is common to diagnostic studies due to differences in patient, study and test characteristics [31]. In this meta-analysis, it is very plausible that part of the heterogeneity is caused by a large variation in morphologic criteria for determination of lymph node metastases. The morphologic and size criteria used are summarized in Table 2, and it is clear that different studies used very different criteria. It is also important to realize that size criteria for US are relevant to differentiate between benign and malignant lymph nodes, and size criteria for USgFNAC are only used to decide whether to puncture or refrain from puncture a lymph node. With respect to the size criteria, there was less variability between studies; most studies used a cut-off size for malignancy of $1 \mathrm{~cm}$ or larger, but one can question whether this commonly used size criterion is a sensible choice. Regarding diameters of $6 \mathrm{~mm}$ or more as suspicious, as was done by Takes and van den Brekel, might better serve the purpose of ruling out occult metastases [11] and [17].

We refrained from scoring the quality of the individual studies, because this issue is still a matter of debate. Whereas incorporation of quality scores is advocated by some [32],others are more critical and point out that the incorporation of quality scores as weights in meta-analysis lacks statistical or empirical justification [33,34]. The alternative approach for assessment of the effect of methodological issues on study results, metaregression analysis, was not feasible due to the small number of studies within each diagnostic modality.

In the current study we evaluated the diagnostic performance of USgFNAC, US, $\mathrm{CT}$ and MRI as they are used in clinical practice to answer the question of cervical lymph node metastases. All of these techniques rely on lymph node size and shape criteria to determine if lymph nodes are abnormal. With ongoing technical developments, additional criteria are evolving that may improve the diagnostic performance of existing techniques. Positron emission tomography (PET) imaging is believed that it may be a valuable addition to the diagnostic armamentarium of the radiologist. However, so far sparse literature where PET imaging alone as a screening tool in the $\mathrm{N} 0$ neck shows a very low sensitivity and no additional value compared to other imaging techniques [35].

In conclusion, this meta-analysis shows that at present USgFNAC is the most reliable imaging technique to assess the presence of metastases in cervical lymph nodes in patients with head and neck cancer. This review also indicates that further studies on diagnostic performances of radiological imaging modalities should be done, focused on patients with clinically N0 necks, because especially in these patients imaging may play an important role in directing treatment. 


\section{References}

1. $\quad$ van de Velde C. Oncologie. Zesde Herziene Druk 2001:1326-39.

2. Foote RL, Olsen KD, Davis DL. Base of tongue carcinoma: patterns of failure and predictors of recurrence after surgery alone. Head Neck 1993;15(4):300-7.

3. Leemans CR, Tiwari R, Nauta JJ, van der Waal I, Snow GB. Recurrence at the primary site in head and neck cancer and the significance of neck lymph node metastases as a prognostic factor. Cancer 1994;73(1): 187-90.

4. Taylor JM, Mendenhall WM, Lavey RS. Time-dose factors in positive neck nodes treated with irradiation only. Radiother Oncol 1991;22(3): 167-73.

5. Ferlito A, Rinaldo A, Robbins KT, et al. Changing concepts in the surgical management of the cervical node metastasis. Oral Oncol 2003; 39(5):429-35.

6. Olsen KD, Caruso M, Foote RL. Primary head and neck cancer. Histopathologic predictors of recurrence after neck dissection in patients with lump node involvement. Arch Otolaryngol Head Neck Surg 1994;120(12):1370-4.

7. Byers RM, Wolf PF, Ballantyne AJ. Rationale for elective modified neck dissection. Head Neck Surg 1988;10(3):160-7.

8. Reitsma JB, Glas AS, Rutjes AW,Scholten RJ, Bossuyt PM, Zwinderman AH. Bivariate analysis of sensitivity and specificity produces informative summary measures in diagnostic reviews. J Clin Epidemiol 2005;58(10):982-90.

9. Moses LE, Shapiro D, Littenberg B. Combining independent studies of a diagnostic test into a summary ROC curve: data-analytic approaches and some additional considerations. Stat Med 1993;12(14):1293-316.

10. Haberal I, Celik H, Gocmen H, Akmansu H, Yoruk M, Ozeri C. Which is important in the evaluation of metastatic lymph nodes in head and neck cancer:palpation, ultrasonography, orcomputedtomography? Otolaryngol Head Neck Surg 2004;130(2):197-201.

11. van den Brekel MW, Castelijns JA, Snow GB. The size of lymph nodes in the neck on sonograms as a radiologic criterion for metastasis: how reliable is it? AJNR Am J Neuroradiol 1998;19(4):695-700.

12. Takeuchi Y, Suzuki H, Omura K, et al. Differential diagnosis of cervical lymph nodes in head and neck cancer by ultrasonography. Auris Nasus Larynx 1999;26(3):331-6.

13. Danninger R, Posawetz W, Humer U, Stammberger H, Jakse R. Ultrasound investigation of cervical lymph node metastases: conception and results of a histopathological exploration. Laryngorhinootologie 1999;78(3): 144-9.

14. Steinkamp HJ, Mathe F, Treisch J, Hosten N, Neumann K, Felix R. A histologically controlled study of the value of sonography and palpation for the detection and exclusion of neck lymph node enlargements and metastases. Aktuelle Radiol 1991;1(6):312-8.

15. Steinkamp HJ, Zwicker C, Langer M, et al. Reactive enlargement of cervical lymph nodes and cervical lymph node metastases: sonography (M/Qquotient) and computed tomography. Aktuelle Radiol 1992;2(4): 188-95.

16. Knappe M, Louw M, Gregor RT. Ultrasonography-guided fine-needle aspiration for the assessment of cervical metastases. Arch Otolaryngol Head Neck Surg 2000;126(9):1091-6.

17. Takes RP, Righi P, Meeuwis CA, et al. The value of ultrasound with ultrasound-guided fineneedle aspiration biopsy compared to computed tomography in the detection of regional metastases in the clinically negative neck. Int J Radiat Oncol Biol Phys 1998;40(5):1027-32.

18. Curtin HD, Ishwaran H, Mancuso AA, Dalley RW, Caudry DJ, McNeil BJ. Comparison of CT and MR imaging in staging of neck metastases. Radiology 1998;207(1):123-30. 
19. McGuirt WF, Williams III DW, Keyes Jr JW, et al. A comparative diagnostic study of head and neck nodal metastases using positron emission tomography. Laryngoscope 1995;105(4 Pt 1):373-5.

20. Carvalho P, Baldwin D, Carter R, Parsons C. Accuracy of CT in detecting squamous carcinoma metastases in cervical lymph nodes. Clin Radiol 1991;44(2):79-81.

21. Freire AR, Lima EN, Almeida OP, Kowalski LP. Computed tomography and lymphoscintigraphy to identify lymph node metastases and lymphatic drainage pathways in oral and oropharyngeal squamous cell carcinomas. Eur Arch Otorhinolaryngol 2003;260(3):14852.

22. Braams JW, Pruim J, Freling NJ, et al. Detection of lymph node metastases of squamous-cell cancer of the head and neck with FDG-PET and MRI. J Nucl Med 1995;36(2):211-6.

23. Anzai Y, Blackwell KE, Hirschowitz SL, et al. Initial clinical experience with dextran-coated superparamagnetic iron oxide for detection of lymph node metastases in patients with head and neck cancer. Radiology 1994;192(3):709-15.

24. Feinmesser R, Freeman JL, Noyek AM, Birt D, Gullane P, Mullen JB. MRI andneckmetastases:aclinical,radiological,pathologicalcorrelativestudy.

J Otolaryngol 1990;19(2):136-40.

25. Sigal R, Vogl T, Casselman J, et al. Lymph node metastases from head and neck squamous cell carcinoma: MR imaging with ultrasmall superparamagnetic iron oxide particles (Sinerem MR)results of a phase-III multicenter clinical trial. Eur Radiol 2002;12(5):1104-13.

26. van den Brekel MW, Castelijns JA, Stel HV, Golding RP, Meyer CJ, Snow GB. Modern imaging techniques and ultrasound-guided aspiration cytology for the assessment of neck node metastases: a prospective comparative study. Eur Arch Otorhinolaryngol 1993;250(1):11-7.

27. Weiss $\mathrm{MH}$, Harrison LB, Isaacs RS. Use of decision analysis in planning a management strategy for the stage N0 neck. Arch Otolaryngol Head Neck Surg 1994;120(7):699-702.

28. Pewsner D, Battaglia M, Minder C, Marx A, Bucher HC, Egger M. Ruling a diagnosis in or out with "SpPIn" and "SnNOut": a note of caution. BMJ 2004;329(7459):209-13.

29. Nieuwenhuis EJ, Castelijns JA, Pijpers R, et al. Wait-and-see policy for the N0 neck in earlystage oral and oropharyngeal squamous cell carcinoma using ultrasonography-guided cytology: is there a role for identification of the sentinel node? Head Neck 2002;24(3):282-9.

30. van den Brekel MW, Castelijns JA, Stel HV, et al. Occult metastatic neck disease: detection with US and US-guided fine-needle aspiration cytology. Radiology 1991;180(2):457-61.

31. Lijmer JG, MolBW, Heisterkamp S, et al. Empirical evidence of design-related bias in studies of diagnostic tests. JAMA 1999;282(11): 1061-6.

32. Whiting P, Rutjes AW, Dinnes J, Reitsma JB, Bossuyt PM, Kleijnen J. A systematic review finds that diagnostic reviews fail to incorporate quality despite available tools. J Clin Epidemiol 2005;58(1):1-12.

33. Juni P,Witschi A, Bloch R, Egger M. The hazards of scoring the quality of clinical trials for meta-analysis. JAMA 1999;282(11):1054-60.

34. Detsky AS, Naylor CD, O'Rourke K, McGeer AJ, L'Abbe KA. Incorporating variations in the quality of individual randomized trials into meta-analysis. J Clin Epidemiol 1992;45(3):25565 .

35. Brouwer J, de Bree R, Comans EF, Castelijns JA, Hoekstra OS, Leemans CR. Positron emission tomography using $[18 \mathrm{~F}]$ fluorodeoxyglucose (FDGPET) in the clinically negative neck: is it likely to be superior? Eur Arch Otorhinolaryngol 2004;261(9):479-83. 


\title{
CHAPTER 4
}

Clinical impact of STIR MRI on staging and management in patients with cervical lymph node metastases of head and neck squamous cell carcinomas.

\author{
B.J. de Bondt \\ R.J. Stokroos \\ J.W. Casselman \\ J.M.A. van Engelshoven \\ R.G.H. Beets-Tan \\ F.G.H. Kessels
}

Head and Neck 2009 Jul; 31(7): 928-37 


\begin{abstract}
Background: We investigated the incremental diagnostic value of Short Tau Inversion Recovery (STIR) MRI to detect cervical nodal metastases in HNSCC.

Methods: Thirty-six patients with cervical nodal metastases underwent MR imaging preceding neck dissection. Two readers evaluated MRI versus MRI with STIR. Level based analysis was performed: inter-observer agreements (Kappa) for detecting normal and metastatic lymph nodes; sensitivities and specificities for detecting at least one metastatic lymph node per level; linear regression analysis to determine performances of MRI with STIR in detecting correct numbers of normal and metastatic lymph nodes. Histopathology was the reference standard.

Results: 175 neck levels were evaluated. MRI with STIR showed better Kappa's for metastatic and normal lymph nodes, was more accurate to estimate numbers of metastatic and normal lymph nodes, and showed improvement of sensitivities and specificities.
\end{abstract}

Conclusion: Incorporation of STIR into the conventional MR protocol improves significantly the detecting of cervical lymph node metastases. 


\section{Introduction}

The presence of cervical metastatic lymph nodes is one of the most important predictors of poor prognosis in patients with head and neck malignancies. The presence of one isolated metastatic lymph node (stage N1) decreases the 5 years survival with $50 \%$, while multiple metastatic lymph nodes decrease the prognosis even more dramatically [1-7].

The extent of known metastatic lymph nodes in the neck influences the choice of management. Aneck dissection may be performed in which one or more lymph node levels are dissected either unilateral or bilateral. Surgery may be followed by radiation therapy depending on histological findings like extra nodal spread, perineural growth and vessel-invasion, or the presence of multiple metastases. All of these therapies carry a considerable morbidity and mortality [8-15]. Thus, radiological imaging plays an important role in the assessment of lymph node involvement as it is used to support treatment decisions; i.e. the extent and laterality of a neck dissection. Furthermore, as metastatic lymph nodes in level IV are correlated with a higher prevalence of distant metastases, i.e. pulmonary metastases, adequate additional pulmonary imaging may play a role in planning a treatment strategy $[16,17]$.

Magnetic Resonance Imaging (MRI), with its excellent soft tissue contrast capabilities over other radiological imaging modalities, is a well established tool to assess lymph nodes. However, despite developments in high-resolution imaging and the development of specific contrast agents for MRI, the detection of small metastases in lymph node remains a difficult task [18-21].

In the work up of patients presenting with a cervical metastatic lymph node of a clinically unknown head and neck squamous cell carcinoma, our MRI protocol included the reversed Short Tau Inversion Recovery (STIR) sequence. Besides the location of the primary tumor, also metastatic lymph nodes showed a marked hypo-intense signal intensity compared to normal lymph nodes. Tumor infiltration with architectural distortion of lymph node parenchyma, necrosis and edema result in areas of proton richness. This proton richness will provide in a longer T1 relaxation time and shortening in T2 relaxation time which results on the reversed STIR sequence in a hypo-intense signal intensity compared to normal tissue. 
In the neck, with its dense lymphatic system, an accurate lesion-by-lesion analysis with pathologic correlation is not always achievable. Therefore, to stage the neck, a level-based classification is used in this study [22]. This classification provides a functional staging, based on the observation of tumor spread to the different lymph node groups.

The aim of this study was to determine the additional value of STIR over conventional MRI for the detection of cervical metastatic lymph nodes in HNSCC.

\section{Materials and Methods}

\section{Patients}

Thirty-six consecutive patients with fine needle aspiration cytology (FNAC) proven metastatic lymph nodes of a clinically unknown primary head and neck squamous cell carcinoma (HNSCC) were studied. The average age was 54.6 years (range $40-80$ years), there were 27 (75\%) men and 9 (25\%) women. A head and neck surgeon performed the otolaryngological examinations, which were followed by magnetic resonance imaging (MRI). Subsequently, all patients underwent a one-sided radical neck dissection. The patients had no history of a previous malignancy and primary or metastatic pulmonary malignancy was ruled out by means of a chest $\mathrm{x}$-ray or chest CT scan.

\section{Imaging}

MR acquisitions were performed according to a standardized protocol on a $1.5 \mathrm{~T}$ MRI scanner (Magnetom 63 SP4000, Siemens, Erlangen, Germany) using a neck coil (Neck Array, Siemens, Erlangen, Germany). The sequence protocol consisted of a coronal short tau inversion recovery (STIR) sequence. This was followed by acquisition of $T_{2}$ weighed (W) images in the axial plane, and fat-saturated $T_{1} W$ images pre- and post intravenous injection of gadolinium-DTPA in the axial and coronal plane. The sequence parameters are listed in table 1 .

\section{Level classification of the neck}

The neck was subdivided according to the six different levels in agreement with the classification of the AJCC (1997) [22]. When viewing the coronal MR images, a reference line on the axial MR images was used to determine the exact neck level. The classification of the neck levels was used by the radiologists when viewing the MR images and used by the pathologist when interpreting the specimen after neck 
dissection; the exact location of each lymph node per neck level was used to match the radiologically examined and pathologically isolated lymph nodes.

\section{Pathological examination}

All neck dissections were performed "en bloc". Immediately after the resection the surgeon positioned the neck dissection specimen on a schematic drawing of the neck in real proportions and fixed it with needles. In the schematic drawing the neck node levels were drawn in. The pathologist manually identified and localized the lymph nodes per neck level in the specimen, the exact location of each lymph node per neck level was matched with the radiologically assessed lymph nodes.

Subsequently, lymph nodes were fixed, sectioned and hematoxyline-eosine (HE) stained. The presence of tumor in each lymph node was examined macroscopically and microscopically. The short-axial diameters of all lymph nodes were noted. The pathological results were used as the reference standard.

\section{$M R$ reading}

Two head and neck radiologists (experience 10 and 12 years in daily interpretation of head and neck imaging) independently interpreted the MR images. The first reading was the conventional MRI scan, subsequently followed by the reading in combination with the STIR sequence.

To suppress the background, the STIR images were evaluated in the reversed mode in which proton rich and pathological tissue show a hypo-intense signal intensity. Lymph nodes were identified as discrete round or oval shaped structures of moderate to high signal intensity on $T_{2} W$ images, iso-intense to muscle on $T_{1} W$ images and hyper-intense on the fat-saturated post contrast $\mathrm{T}_{1} \mathrm{~W}$ images.

The number of normal lymph nodes and metastatic lymph nodes were separately registered per level as stated earlier in the section "level classification of the neck". The index lymph node was registered; this was defined as the metastatic lymph node the patients initially presented with. Besides the knowledge of a metastatic lymph node of an unknown primary head and neck tumor, both readers were blinded for any other clinical information and each other assessments of the lymph nodes.

The criterion for lymph node involvement on $T_{1} W$ and $T_{2} W$ images was the short axial diameter, defined as the minimal axial diameter in the largest region of the 
lymph node, in which the cut-off point to classify lymph nodes as abnormal was $>10 \mathrm{~mm}$.

The criterion for lymph node involvement on the STIR sequence accounting for lymph nodes of all sizes was the signal intensity on the reversed images in which hypo-intense signal intensity was considered pathological and suggestive of presence of metastasis.

\section{Statistical analysis}

Inter observer agreement for detecting normal lymph nodes as well as metastatic lymph node was determined per neck level by using quadratic weighted kappa's. The difference in kappa's between the regular MRI and the combination of MRI with STIR was tested using a bootstrap technique [23, 24].

For each level, sensitivities and specificities for detecting at least one metastatic lymph node were determined for both observers. The pathological results were the reference standard. The p-values for differences in sensitivity and specificity between MRI and the combination of MRI with STIR were tested with the $\mathrm{McNemar}$ test in which a p-value below 0.05 was taken to indicate significance.

In addition, the performance of MRI and the combination of MRI with STIR in detecting the correct number of lymph nodes and the correct number of metastases was determined with a linear regression model. The difference in fit as expressed by the $\mathrm{R}^{2}$ between the two test modalities was tested with a bootstrap technique. A pvalue below 0.05 was taken to indicate significance.

Table 1. Sequence parameters of the head and neck MRI* protocol.

\begin{tabular}{|c|c|c|c|c|}
\hline Parameter & $\mathrm{STIR}^{\dagger}$ & SPIR T ${ }^{2} \mathrm{~W}$ & $\mathrm{~T}^{1} \mathrm{~W}$ & SPIR CE T1W \\
\hline & (coronal) & (axial) & (axial, coronal) & (axial, coronal) \\
\hline $\mathrm{TE} / \mathrm{TR} / \mathrm{TI}^{\ddagger}(\mathrm{ms})$ & $60 / 5868 / 150$ & $80 / 1704 /-$ & $12 / 400 /-$ & $12 / 740 /-$ \\
\hline Matrix & 256 & 256 & 256 & 256 \\
\hline Slice/gap thickness $(\mathrm{mm})$ & $3 / 0$ & $3 / 0$ & $3 / 0$ & $3 / 0$ \\
\hline Field of view (mm) & 160 & 160 & 160 & 160 \\
\hline $\mathrm{NSA}^{\mathrm{S}}$ & 2 & 2 & 2 & 2 \\
\hline Scan time $(\mathrm{min})$ & $3: 37$ & $3: 21$ & $2: 37$ & $4: 47$ \\
\hline
\end{tabular}

${ }^{*}$ Magnetic Resonance Imaging, $\dagger$ Short Tau Inversion Recovery, $\otimes$ Spectral Pre-saturation Inversion Recovery T2 weighted image, 9 T1 weighted image, \# SPIR contrast enhanced T1 weighted image, Echo Time/Repetition Time/Inversion Time, $\$$ Number of Signals Averaged 


\section{Results}

Thirty-six specimens were pathologically examined representing 180 neck levels with a total of 962 lymph nodes; in 156 lymph nodes metastasis could be demonstrated.

Table 2 presents the inter observer agreement $(\kappa)$ ofthe two observers per lymph node level. For detecting metastasis with conventional MRI, $\kappa$ was best for level I $(\kappa=0.79)$ and least for level III $(\kappa=0.46)$. When MRI was combined with STIR, the approaches 1 for all levels in the neck ( $\kappa$ ranged from 0.89-0.99). Differences in $\kappa$ was only statistically significant for level I, II and III. Regarding accurate assignment of lymph nodes as being normal on conventional MRI, the $\kappa$ ranged from 0.73-0.92. MRI combined with STIR resulted in a $\kappa$ range from 0.85-0.99. No statistically significant differences were found in any level of the neck.

Table 3 presents the sensitivities and specificities for both observers for the correct diagnosis of at least one metastatic lymph node per neck level. Differentiation has been made with absolute numbers of patients with positive $\left(\mathrm{N}_{+}\right)$and negative (N0) neck levels at pathological examination. Sensitivities in the $\mathrm{N}+$ necks levels for conventional MRI and MRI combined with STIR ranged from $25-96 \%$ and $93-$ $100 \%$, respectively, and specificities in the N0 neck levels ranged from $20-100 \%$ and $96-100 \%$, respectively. For both observers, the differences in sensitivity were only statistically significant for level III and IV, and for the specificity this accounted only for level III.

Table 4 shows the slopes of the regression equations averaged over the 5 evaluated levels in the neck. For both observers, MRI overestimates the number of normal lymph nodes as well as the number of metastatic lymph nodes (slopes $>1$ ). In contrast, the prediction of the number of normal lymph nodes and metastatic lymph nodes on MRI combined with STIR is more accurate (slopes $\approx 1$ ).

Furthermore, when the conventional MRI is combined with STIR, the predictions of metastatic lymph nodes and normal lymph nodes are significantly more precise as tested by the differences in $\mathrm{R}^{2}$. 
Table 2. Inter observer agreements $(\kappa)$ of the two observers for the detection of metastatic lymph nodes and normal lymph nodes by means of conventional MRI* alone and MRI combined with the STIR† sequence.

\begin{tabular}{lllllll}
\hline Level & \multicolumn{3}{l}{ Metastatic lymph nodes } & \multicolumn{3}{l}{ Normal lymph nodes } \\
& MRI & MRI + STIR & p-value & MRI & \multicolumn{2}{c}{ MRI + STIR p-value } \\
\hline I & 0.79 & 1 & $<0.001$ & 0.89 & 099 & 0.056 \\
II & 0.67 & 0.99 & 0.009 & 0.73 & 0.96 & 0.29 \\
III & 0.46 & 0.97 & 0.003 & 0.93 & 0.96 & 0.58 \\
IV & 0.65 & 0.95 & 0.027 & 0.89 & 0.85 & 0.56 \\
V & 0.62 & 0.89 & 0.17 & 0.92 & 0.95 & 0.28 \\
\hline
\end{tabular}

${ }^{*}$ Magnetic Resonance Imaging, ${ }^{\dagger}$ Short Tau Inversion Recovery

Table 3. Sensitivities and specificities of the two observers for the detection of at least one metastatic lymph node per level in the neck by means of conventional MRI* alone and MRI combined with the STIR† sequence.

\begin{tabular}{|c|c|c|c|c|c|c|c|c|c|}
\hline \multirow{3}{*}{ Level } & \multirow{3}{*}{$\begin{array}{c}\text { Pathol. } \\
\mathrm{N}+\text { level (n) }\end{array}$} & \multicolumn{3}{|c|}{ ObserverSensitivity Sensitivity } & \multirow[t]{3}{*}{$\mathrm{p}$-value } & \multirow{3}{*}{$\begin{array}{l}\text { Pathol. } \\
\text { No level (n) }\end{array}$} & \multicolumn{3}{|c|}{ Specificity Specificity p-value } \\
\hline & & & $(\%)$ & $(\%)$ & & & $(\%)$ & $(\%)$ & \\
\hline & & & MRI & $\begin{array}{c}\mathrm{MRI}+ \\
\text { STIR }\end{array}$ & & & MRI & $\begin{array}{c}\text { MRI + } \\
\text { STIR }\end{array}$ & \\
\hline \multirow[t]{2}{*}{ I } & 4 & I & 25 & 100 & 0.26 & 32 & 100 & 100 & 1 \\
\hline & & II & 25 & 100 & 0.26 & & 100 & 100 & 1 \\
\hline \multirow[t]{2}{*}{ II } & 25 & I & 96 & 100 & 1 & 11 & 73 & 100 & 0.26 \\
\hline & & II & 96 & 100 & 1 & & 73 & 100 & 0.26 \\
\hline \multirow[t]{2}{*}{ III } & 21 & I & 71 & 100 & 0.03 & 15 & 60 & 100 & 0.03 \\
\hline & & II & 76 & 100 & 0.06 & & 20 & 100 & 0.0005 \\
\hline \multirow[t]{2}{*}{ IV } & 15 & I & 47 & 100 & 0.008 & 21 & 81 & 100 & 0.13 \\
\hline & & II & 53 & 93 & 0.03 & & 81 & 100 & 0.13 \\
\hline \multirow[t]{2}{*}{$\mathrm{V}$} & 9 & I & 44 & 100 & 0.06 & 22 & 100 & 96 & 1 \\
\hline & & II & 57 & 100 & 0.13 & & 96 & 100 & 1 \\
\hline
\end{tabular}

${ }^{*}$ Magnetic Resonance Imaging, ${ }^{\dagger}$ Short Tau Inversion Recovery

Table 4. Slopes of the linear regression model predicting the number of normal lymph nodes and lymph node metastases, averaged over the 5 levels in the neck for MRI* and MRI combined with STIR $†$ for both observers, and p-values for the difference in fit as expressed by R2 of the model.

\begin{tabular}{|c|c|c|c|c|c|c|}
\hline \multirow[t]{3}{*}{ Observer } & \multicolumn{3}{|c|}{ Metastatic lymph nodes } & \multicolumn{3}{|c|}{ Normal lymph nodes } \\
\hline & \multirow[t]{2}{*}{ slope MRI } & \multicolumn{2}{|c|}{ slope MRI + p-value as a } & \multirow[t]{2}{*}{ slope MRI } & \multicolumn{2}{|c|}{ slope MRI + p-value as a } \\
\hline & & STIR & difference of $\mathrm{R}^{2}$ & & STIR & difference of $\mathrm{R}^{2}$ \\
\hline I & 1.60 & 1.06 & 0.0006 & 1.21 & 1.07 & 0.002 \\
\hline II & 1.24 & 1.12 & $<0.0001$ & 1.25 & 1.04 & 0.002 \\
\hline
\end{tabular}

${ }^{\circ}$ Magnetic Resonance Imaging, ${ }^{\dagger}$ Short Tau Inversion Recovery 

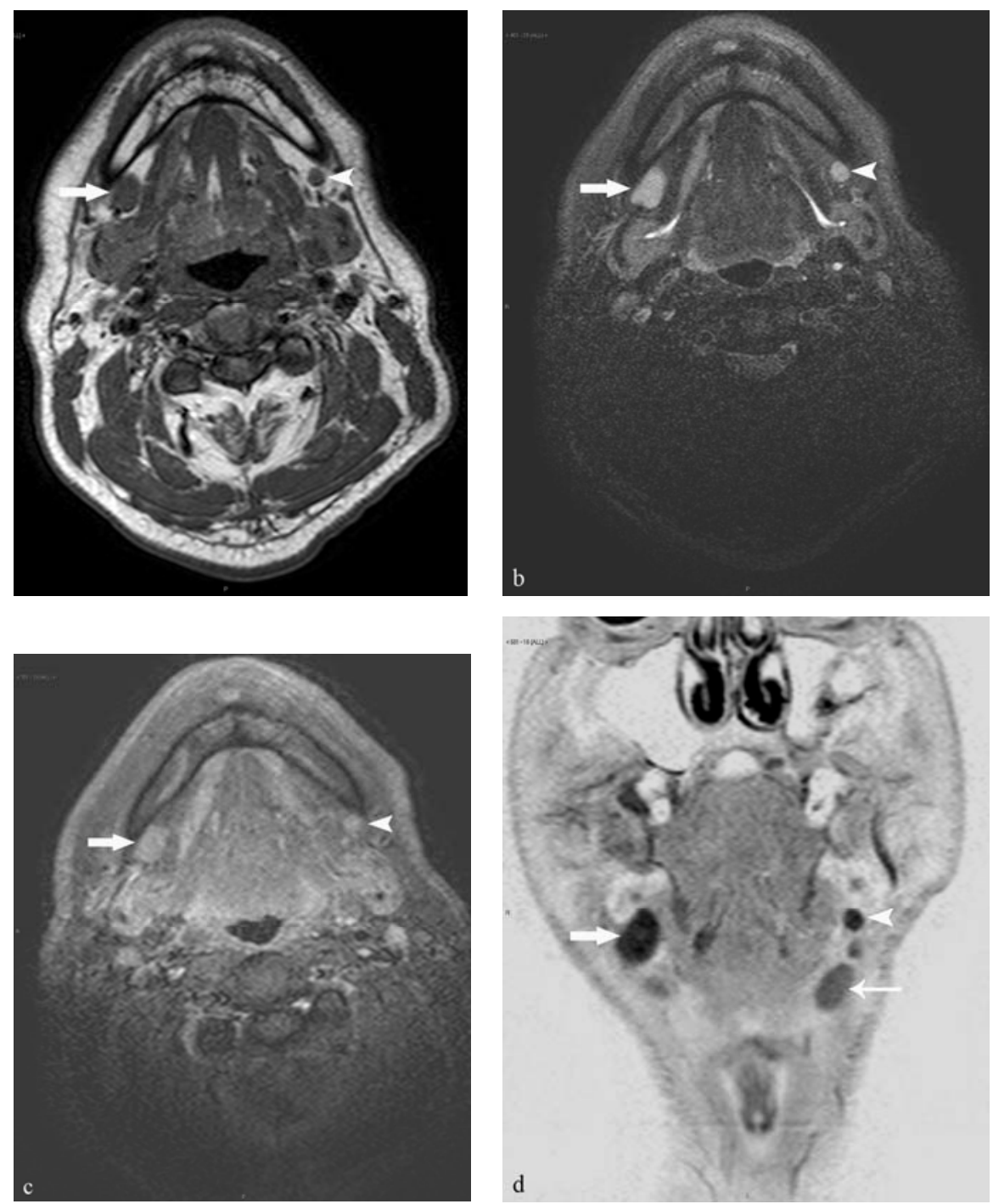

Figure 1a-d. MR images of the neck of a patient with right sided cervical lymph node metastasis of an unknown primary tumor. Axial T1W image (a) and fat-saturated T2W image (b) show in level Ia on the right side a pathological enlarged lymph node (arrow) and on the left side an ovoid lymph node (arrowhead) with $5 \mathrm{~mm}$ short axial diameter with normal appearance, there is normal enhancement on the contrast enhanced fat-saturated T1W image (c). On the corresponding coronal reversed STIR image (d), the same small lymph node (arrowhead) shows similar pathological hypo-intense signal intensity (SI) compared to the pathological lymph node on the right side (arrow): ultrasound guided fine needle aspiration cytology (USgFNAC) confirmed the diagnosis of metastases of squamous cell carcinoma (SCC) in both lymph nodes. Note, in contrast, the enlarged lymph node (small arrow) on the left side in level Ia with normal signal intensity on the STIR in which USgFNAC revealed no SCC. 

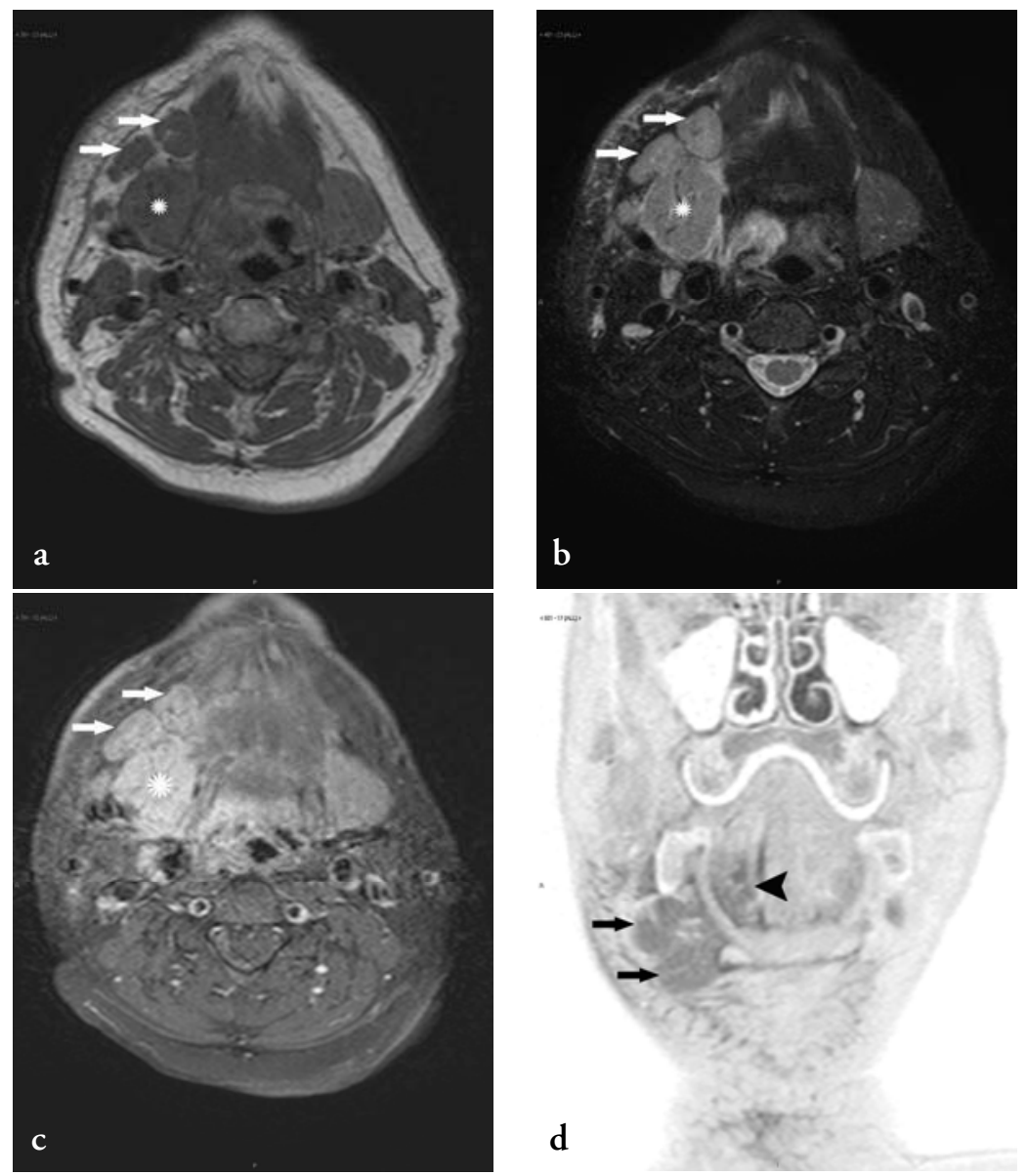

Figure 2a-d. MR images of the neck in a patient presenting with enlarged lymph nodes on the right side. The axial T1W image (a), fat-saturated T2W image (b) and contrast enhanced fat-saturated T1W image (c) show two pathological enlarged lymph nodes in level Ia on the right side (arrows). The corresponding coronal reversed STIR image (d) demonstrates the same enlarged lymph nodes (arrows) showing normal gray signal intensity compared to other non-enlarged lymph nodes. Ultrasound guided fine needle aspiration cytology (USgFNAC) revealed no SCC. The asterix indicates obstructive enlargement of the submandibular gland on the right side. The arrowhead indicates edema surrounding a small primary tumor in the floor of the mouth. 


\section{Discussion}

This study demonstrates that adding short tau inversion recovery (STIR) to the conventional magnetic resonance imaging (MRI) protocol improves the detection of cervical lymph node metastases significantly in patients with head and neck squamous cell carcinoma (HNSCC).

A recently published meta-analysis of radiological studies evaluating ultrasound (US), ultrasound guided fine needle aspiration cytology (USgFNAC), MRI and computed tomography (CT) for the detection of neck node metastases, presented sensitivities ranging from $48-97 \%$ and specificities ranging from $39-96 \%$ [25]. When focused on MRI studies only, sensitivity and specificity ranged from 67$92 \%$ and $40-94 \%$, respectively [18-21]. In these studies a large variety of size criteria and morphological criteria were used to assess lymph nodes; the long or short axial diameter, or ratio of the short and long axial diameter, and also morphological criteria such as central necrosis, extra nodal tumor spread, heterogeneity and contrast enhancement were mentioned. However, the usefulness of the short axial diameter as size criterion, and heterogeneity of lymph nodes as morphological criterion have been established [18, 26-28]. Central necrosis and extra nodal tumor spread appear to be a biologically late event in the development of tumor in lymph nodes and is mainly seen in lymph nodes exceeding the $10 \mathrm{~mm}$ in short axial diameter [29]. However, these lymph nodes in this presented study will be classified as metastatic anyway based on the short axial diameter, and one could question the added value over the size criterion.

In this study we have chosen for the established and generally accepted short axial diameter as size criterion for evaluating lymph nodes on the conventional MRI. On the level based analysis for the detection of at least one metastatic lymph node per neck level, sensitivity and specificity for the different levels for the conventional MRI ranged from $25-96 \%$ and $20-100 \%$, respectively. However, for MRI combined with STIR sensitivity and specificity ranged even from $93-100 \%$ and 96-100\%, respectively.

There are some important clinical implications of this more accurate imaging technique that have to be addressed. Firstly, a more reliable level based classification for cervical lymph node metastases can be achieved; the more exact determination of the location of metastatic lymph nodes in the neck levels will allow the surgeon to dissect only the levels involved in metastatic disease. Secondly, accurate assessment of dissemination of metastases in the neck levels could also guide the radiotherapist in determining the field of radiation. One has to remind 
that both surgical and radiation treatment refinements will limit patient morbidity [1-7]. The nodal $(\mathrm{N})$ status, as part of the TNM staging system, is an important prognostic factor in head and neck cancer in predicting the survival rate and therefore influences the choice of treatment [8-15].

Another advantage of STIR is the rapid imaging technique, it will increase the scan time with only 3 to 4 minutes. Furthermore, adding STIR to the MRI protocol is applicable in all MR scanners, the reading of the images is relatively easy also for radiologists not experienced in head and neck radiology. An advantage of STIR viewing in the reversed mode is the suppression of the background, pathological lymph nodes will pop-up more easily and the observer is less distracted by the surrounding tissue. It is important to note that this phenomenon was observed not only in large metastases but also in metastatic lymph nodes as small as $5 \mathrm{~mm}$, this is illustrated in a small ovoid lymph node in figure 1. In contrast, figure 2 shows enlarged lymph nodes a patient initially presented with, these lymph nodes would be classified as pathologic based on the short axial diameter. However, STIR demonstrates a normal gray signal-intensity and cytological examination revealed no metastases.

It has to be emphasized that the sole finding of pathological hypo-intense signal on the reversed STIR images will not be sufficient to determine lymph nodes as malignant; STIR is highly sensitive for the detection of pathologic lesions, but it is not specific for malignancy alone whereas the same phenomenon could be observed for example in inflammatory lymph nodes. However, in patients with head and neck cancer, this finding on STIR is an important indicator of a pathological lymph node in which the other MRI sequences should be reviewed for the decision to determine a lymph node to be malignant.

Other reports presented the usefulness of STIR for the detection of a variety of malignancies by using this sequence as a screening tool for whole body imaging [30-33]. Only a few studies focused on the detection of metastatic lymph in the head and neck. Brüning et al. already assessed in 1994 the value of STIR for the demarcation of primary head and neck tumors [34]. Müller-Lisse et al. reported the STIR sequence as a pathfinder for primary tumors and lymph nodes in the head and neck, however, there was no pathological correlation [35]. Another study reported SPECT versus STIR for the detection of metastatic lymph nodes in HNSCC. This level based analysis with pathological correlation showed a sensitivity and specificity of $92 \%$ and $71 \%$, respectively [36]. Nakatsu et al. published a descriptive report in which STIR was compared with other fat suppression MR sequences for lesions in the neck and thorax, however, they did 
not focus on lymph nodes and there was no pathological correlation [37]. Kawai et al. recently reported the results of STIR for the detection of cervical metastatic lymph nodes, they advocated STIR to use as a screening technique for cervical metastatic lymph nodes prior to further detailed MR study of the neck [38].

Although adding STIR to the protocol will increase the total scan time with only a few minutes, one could question whether it is justified to adjust the MRI protocol by withdrawing a sequence that has only little added value. This issue will remain matter for further studies.

There are some short comings when focused on this study that have to be mentioned. Firstly, the issue of the small series, patients presenting with a metastatic lymph node of an unknown primary HNSCC is infrequent compared to the population of patients presenting with a primary head and neck tumor in which metastatic lymph nodes may be present. Secondly, in this respect we have to mention that only patients with positive necks $\left(\mathrm{N}_{+}\right)$were evaluated in this study, as they initially presented with a neck node metastasis; the knowledge of the presence of at least one metastastic lymph node might influence the observers to assign more easily other lymph nodes as metastatic. The question raises whether the presented results could be extrapolated to other patient groups, and whether we expect the same results in the clinically negative neck (N0). Thirdly, due to the large number of lymph nodes in the neck, a well-known disadvantage of the staging system is the difficulty of a true lesion-by-lesion analysis. Therefore, to stage the neck, we used the level-based classification. Consequently, uncertainty whether lymph nodes truly match the pathologically examination will unfortunately remain.

\section{Conclusion}

In this study we have demonstrated the incremental impact of STIR on staging and subsequently on management of patients with cervical lymph node metastases. STIR is a sensitive and fast MRI sequence and adding STIR to the conventional MRI protocol is strongly recommended. 


\section{References}

1. Ono I, Ebihara S, Saito H, et al. Correlation between prognosis and degree of lymph node involvement in carcinoma of the head and neck. Auris Nasus Larynx 1985;12 Suppl 2:S85-89.

2. Jakobsen J, Hansen O, Jorgensen $\mathrm{KE}$, et al. Lymph node metastases from laryngeal and pharyngeal carcinomas--calculation of burden of metastasis and its impact on prognosis. Acta Oncol 1998;37:489-493.

3. Kehrl W, Wenzel S, Niendorf A. [Effect of various forms of metastatic lymph node involvement on prognosis of squamous epithelial carcinomas of the upper aerodigestive tract]. Laryngorhinootologie 1998;77:569-575.

4. Ganzer U, Meyer-Breiting E, Ebbers J, et al. [Effect of tumor size on lymph node metastasis and type of treatment on the prognosis of hypopharyngeal cancer]. Laryngol Rhinol Otol (Stuttg) 1982;61:622-628.

5. Leemans CR, Tiwari RM, van der Waal I, et al. [Neck lymph node dissection in squamous cell carcinoma originating in the head-and-neck area; the significance for the prognosis]. Ned Tijdschr Geneeskd 1992;136:221-225.

6. Foote RL, Olsen KD, Davis DL. Base of tongue carcinoma: patterns of failure and predictors of recurrence after surgery alone. Head Neck 1993;15:300-307.

7. Leemans CR, Tiwari R, Nauta JJ, et al. Recurrence at the primary site in head and neck cancer and the significance of neck lymph node metastases as a prognostic factor. Cancer 1994;73:187-190.

8. Taylor JM, Mendenhall WM, Lavey RS. Time-dose factors in positive neck nodes treated with irradiation only. Radiother Oncol 1991;22:167-173.

9. Newman JP, Terris DJ, Pinto HA, et al. Surgical morbidity of neck dissection after chemoradiotherapy in advanced head and neck cancer. Ann Otol Rhinol Laryngol 1997; 106:117-122.

10. Hillel A, Patten C. Neck dissection: morbidity and rehabilitation. Cancer Treat Res 1990;52:133-147.

11. Sanguineti G, Sormani MP, Benasso M, et al. Late local treatment morbidity after accelerated radiotherapy or alternating chemoradiotherapy for advanced head and neck carcinoma. Tumori 2002;88:313-320.

12. Bentzen SM, Saunders MI, Dische S, et al. Radiotherapy-related early morbidity in head and neck cancer: quantitative clinical radiobiology as deduced from the CHART trial. Radiother Oncol 2001;60:123-135.

13. Ferlito A, Rinaldo A, Robbins KT, et al. Changing concepts in the surgical management of the cervical node metastasis. Oral Oncol 2003;39:429-435.

14. Olsen KD, Caruso M, Foote RL. Primary head and neck cancer. Histopathologic predictors of recurrence after neck dissection in patients with lump node involvement. Arch Otolaryngol Head Neck Surg 1994;120:1370-1374.

15. Byers RM, Wolf PF, Ballantyne AJ. Rationale for elective modified neck dissection. Head Neck Surg 1988;10:160-167.

16. de Bree R, Deurloo EE, Snow GB, et al. Screening for distant metastases in patients with head and neck cancer. Laryngoscope 2000;110:397-401.

17. Plaat RE, de Bree R, Kuik DJ, et al. Prognostic importance of paratracheal lymph node metastases. Laryngoscope 2005;115:894-898.

18. Curtin HD, Ishwaran H, Mancuso AA, et al. Comparison of CT and MR imaging in staging of neck metastases. Radiology 1998;207:123-130.

19. Braams JW, Pruim J, Freling NJ, et al. Detection of lymph node metastases of squamous-cell cancer of the head and neck with FDG-PET and MRI. J Nucl Med 1995;36:211-216. 
20. Feinmesser R, Freeman JL, Noyek AM, et al. MRI and neck metastases: a clinical, radiological, pathological correlative study. J Otolaryngol 1990;19:136-140.

21. Sigal R, Vogl T, Casselman J, et al. Lymph node metastases from head and neck squamous cell carcinoma: MR imaging with ultrasmall superparamagnetic iron oxide particles (Sinerem MR) --results of a phase-III multicenter clinical trial. Eur Radiol 2002;12:1104-1113.

22. Fleming I, Cooper J, Henson D. AJCC Staging manual. Lippenscott-Raven 1997;5th ed. Philadelphia.

23. Efron B. An Introduction to the Bootstrap (Monographs on Statistics and Applied Probability). Chapman \& Hall/CRC 1994.

24. Crary SB, Fahey DA. Analysis of critical-exponent data using Efron's bootstrap technique. Phys Rev B Condens Matter 1987;35:2102-2104.

25. de Bondt RB, Nelemans PJ, Hofman PA, et al. Detection of lymph node metastases in head and neck cancer: A meta-analysis comparing US, USgFNAC, CT and MR imaging. Eur J Radiol 2007.

26. van den Brekel MW, Stel HV, Castelijns JA, et al. Cervical lymph node metastasis: assessment of radiologic criteria. Radiology 1990;177:379-384.

27. van den Brekel MW, Castelijns JA, Stel HV, et al. Detection and characterization of metastatic cervical adenopathy by MR imaging: comparison of different MR techniques. J Comput Assist Tomogr 1990;14:581-589.

28. van den Brekel MW, Castelijns JA, Croll GA, et al. Magnetic resonance imaging vs palpation of cervical lymph node metastasis. Arch Otolaryngol Head Neck Surg 1991;117:663-673.

29. Som PM. Lymph nodes of the neck. Radiology 1987;165:593-600.

30. Walker R, Kessar P, Blanchard R, et al. Turbo STIR magnetic resonance imaging as a wholebody screening tool for metastases in patients with breast carcinoma: preliminary clinical experience. J Magn Reson Imaging 2000;11:343-350.

31. Eustace S, Tello R, DeCarvalho V, et al. Whole body turbo STIR MRI in unknown primary tumor detection. J Magn Reson Imaging 1998;8:751-753.

32. Takenaka D, Ohno Y, Hatabu H, et al. Differentiation of metastatic versus non-metastatic mediastinal lymph nodes in patients with non-small cell lung cancer using respiratory-triggered short inversion time inversion recovery (STIR) turbo spin-echo MR imaging. Eur J Radiol 2002;44:216-224.

33. Takahara T, Imai Y, Yamashita $T$, et al. Diffusion weighted whole body imaging with background body signal suppression (DWIBS): technical improvement using free breathing, STIR and high resolution 3D display. Radiat Med 2004;22:275-282.

34. Bruning R, Heuck A, Naegele M, et al. [Fat-suppressing STIR sequences with and without contrast media in the MRT of ENT tumors]. Rofo 1994;160:412-416.

35. Muller-Lisse GU, Kretschmar UL, Jager L, et al. [Value of fat signal suppression MRI pulse sequences for diagnosis of malignant tumors in the area of the head-neck]. Radiologe 1996;36:199-206.

36. Valdes Olmos RA, Koops W, Loftus BM, et al. Correlative 201Tl SPECT, MRI and ex vivo $201 \mathrm{Tl}$ uptake in detecting and characterizing cervical lymphadenopathy in head and neck squamous cell carcinoma. J Nucl Med 1999;40:1414-1419.

37. Nakatsu M, Hatabu H, Itoh H, et al. Comparison of short inversion time inversion recovery (STIR) and fat-saturated (chemsat) techniques for background fat intensity suppression in cervical and thoracic MR imaging. J Magn Reson Imaging 2000;11:56-60.

38. Kawai Y, Sumi M, Nakamura T. Turbo short tau inversion recovery imaging for metastatic node screening in patients with head and neck cancer. AJNR Am J Neuroradiol 2006;27:12831287. 
$\longrightarrow$ 


\title{
CHAPTER 5
}

Morphological MRI criteria improve the detection of lymph node metastases in head and neck squamous cell carcinoma: multivariate logistic regression analysis of MRI features of cervical lymph nodes

\author{
R.B.J. de Bondt \\ P. J. Nelemans \\ F. Bakers \\ J.W. Casselman \\ C. Peutz-Kootstra \\ B. Kremer \\ P.A.M. Hofman \\ R.G.H. Beets-Tan
}

European Radiology 2009 Mar; 19(3): 626-33

Awarded with the annual prize of the Dutch Radiological Society, 2007 


\begin{abstract}
The aim was to evaluate whether morphological criteria in addition to the size criterion results in better diagnostic performance of MRI for the detection of cervical lymph node metastases in patients with head and neck squamous cell carcinoma (HNSCC). Two radiologists evaluated 44 consecutive patients in which lymph node characteristics were assessed with histopathological correlation as gold standard. Assessed criteria were the short axial diameter and morphological criteria such as border irregularity and homogeneity of signal intensity on T2weighted and contrast-enhanced T1-weighted images. Multivariate logistic regression analysis was performed: diagnostic odds ratios (DOR) with 95\% confidence intervals $(95 \% \mathrm{CI})$ and areas under the curve (AUCs) of receiveroperating characteristic (ROC) curves were determined.
\end{abstract}

Border irregularity and heterogeneity of signal intensity on T2-weighted images showed significantly increased DORs. AUCs increased from 0.67 (95\% CI: 0.610.73 ) using size only to 0.81 (95\% CI: $0.75-0.87)$ using all four criteria for observer 1 and from 0.68 (95\% CI: $0.62-0.74)$ to 0.96 (95\% CI: $0.94-0.98)$ for observer $2(p<0.001)$. This study demonstrated that the morphological criteria border irregularity and heterogeneity of signal intensity on T2-weighted images in addition to size significantly improved the detection of cervical lymph nodes metastases. 


\section{Introduction}

The presence of cervical lymph node metastases is an important prognostic factor in patients with HNSCC as it worsens significantly the treatment outcome [1-8]. The choice of management depends on the existence and extent of lymph node metastases in the neck. Therapy could consist of surgery, radiation therapy, chemotherapy, or a combined therapy. However, all these therapies have a considerable morbidity and mortality. Therefore, treatment refinements like limitation of the field of radiation or a more selective neck dissection are necessary [9-15]. MRI is one of the imaging techniques that is used to guide treatment decisions, but the ability of MRI to discriminate between lymph nodes with and without metastasis is still poor [16].

A commonly used criterion for the assessment of cervical lymph node involvement on MRI is the short axial diameter, and several studies have been undertaken to determine the optimal cutoff size of the short axial diameter for discrimination between metastatic and non-metastatic lymph nodes. On MRI a commonly used size cutoff point is a short axial diameter of $10 \mathrm{~mm}$, but a range varying from 9 to $15 \mathrm{~mm}$ has been described [17-21]. The challenge for radiologists remains the detection of metastases in small lymph nodes with a short axial diameter below 10 $\mathrm{mm}$, because mere use of the size criterion will result in misclassification of these nodes as normal. In this respect, according to the literature, the performance of MRI is still poor for detection of lymph node metastases, and this concerns especially the detection of metastases in small lymph nodes [16].

In addition to the size criterion, the use of morphological criteria might have added value to detect metastatic disease in lymph nodes. The diagnostic value of morphological criteria has not been well evaluated in patients with HNSCC. In patients with rectal cancer, Kim et al. demonstrated that in addition to the size criterion, morphological criteria such as borders and heterogeneity could be helpful signs to predict nodal involvement [22].

The aim of our study was to evaluate the accuracy and additional diagnostic value of morphological criteria observed on MRI images, such as border irregularity and heterogeneity of the signal intensity on T2-weighted images and heterogeneity of enhancement on post-contrast T1-weighted images. 


\section{Materials and methods}

\section{Patients}

This study was approved by the local medical ethics committee. Between January 2002 and December 2006 a series of 44 consecutive patients [mean age was 61 years (range: $40-86$ years); 11 women and 33 men] with a HNSCC who underwent a unilateral or bilateral supraomohyoid neck dissection (SOHND) (dissection of levels I-III) or radical (modified) neck dissection (R(M)ND) (dissection of levels I-V) was studied. All patients underwent MR imaging as part of the routine diagnostic workup. This was performed on a 1.5-T machine (Gyroscan, Powertrack 6000, Philips, Best, The Netherlands) by using a head-neck coil (Philips, Best, The Netherlands). Table 1 presents the relevant parameters of the MRI protocol.

\section{MR evaluation}

One general radiologist (observer 1) and one radiologist specialized in head and neck imaging (observer 2) independently evaluated the MR images retrospectively. Both observers were blinded to each other's MR assessments, clinical information, and the results of the histological examination.

In the first session, all lymph nodes were determined by position and slice number per neck level. The short axial diameter was recorded per lymph node. All lymph nodes were classified as normal $(\leq 10 \mathrm{~mm})$ or malignant $(>10 \mathrm{~mm})$.

After a time interval of 8 weeks, the same MR images were reviewed again by the same radiologists blinded to each others' MR assessments, clinical information, and the results of the histological examination. For every lymph node the decision was made whether it was normal or metastatic using three morphologic criteria. The first criterion was border as observed on fat-suppressed T2-weighted images (smooth, lobulated, spiculated, indistinct) (Figure 1 demonstrates the four different borders on the schematic drawing). Smooth (Figure 2A) and lobulated (Figure 2B) borders were considered as indicative of normal lymph nodes. Spiculated (Figure 2C) and indistinct (Figure 2D) borders were considered as indicative of metastatic lymph nodes. The second criterion was degree of homogeneity of the signal intensity on contrast-enhanced T1-weighted images. A homogeneous enhancement was considered as normal. A heterogeneous enhancement was considered as indicative of metastatic lymph nodes. The third criterion was degree of homogeneity of the signal intensity on T2-weighted images. A homogeneous signal intensity was considered as normal. A heterogeneous signal intensity was considered as indicative of metastatic lymph nodes (Figure 3). 


\section{Pathological examination}

All neck dissections were performed en bloc. Immediately after the resection, the surgeon positioned the neck dissection specimen on a schematic drawing of the neck in real proportions and fixed it with needles (Figure 4). The pathologist manually identified and localized the lymph nodes per neck level in the specimen. The short axial diameter of all lymph nodes was recorded. Subsequently, lymph nodes were fixed, sectioned, and hematoxyline-eosine (HE) stained, and the presence of tumor in each lymph node was examined microscopically. The pathological results were used as the reference standard.

\section{Matching MR assessment to pathological examination}

The neck was subdivided according to the six different levels in agreement with the classification of the American Joint Committee on Cancer (AJCC); this classification was used by the radiologists when viewing the MR images and used by the pathologist when interpreting the specimen after neck dissection [23].

The results of the measurements on MRI were compared with the results of the pathologic examination of the neck dissection specimens. By recording the combination of the short axial diameter and the exact location of each lymph node, it was possible to perform a topographical correlation for each lymph node per neck level.

\section{Statistical analysis}

Inter-observer agreement with respect to the evaluation of the criterion nodal size, and the criteria border irregularity, homogeneity of contrast enhancement on T1weighted images, and homogeneity of signal intensity on T2-weighted images was expressed by Cohen's kappa coefficient $(\kappa)$.

For each lymph node, the scores for the MRI criteria were correlated with the outcome of the histological examination of the neck dissection specimen: presence or absence of lymph node metastasis. Diagnostic performances of nodal size and the new criteria for the diagnosis of metastasis were evaluated using diagnostic parameters, such as sensitivity, specificity, and diagnostic odds ratio (DOR). In diagnosis, one always has to compromise between sensitivity and specificity: as sensitivity increases, specificity will decrease and vice versa. The DOR is a measure of diagnostic performance, which incorporates sensitivity and specificity and thus captures the trade-off between these measures. A DOR $=1$ indicates that the test under study has no diagnostic value; a $\mathrm{DOR}>1$ indicates that the test under study (in this case, the new MRI criteria) has the ability to discriminate between lymph 
nodes with and without metastasis. The larger the DOR is, the larger the discriminative ability [24].

Multivariate logistic regression analysis was used to evaluate whether a criterion, when used in combination with the other criteria, can be used as an independent indicator for differentiating metastatic from non-metastatic lymph nodes. The independent contribution of each criterion to the diagnostic performance is expressed as the DOR, which can be derived by exponentiation of the corresponding regression coefficient, where $95 \%$ confidence intervals $(95 \% \mathrm{CI})$ are used to indicate whether the DOR is significantly different from DOR=1. Multivariate logistic regression analysis was also used to examine which of the new MRI criteria were most predictive of the presence of metastases and whether the use of these new criteria in addition to size significantly improved the detection of cervical lymph nodes metastases in HNSCC.

The dependent variable in these models was the presence or absence of metastasis according to the pathological examination. The models incorporated as independent variable size as well as the three new MRI criteria. All criteria were entered as dichotomous variables, where abnormal and normal results were coded as 1 and 0 , respectively.

Predicted probabilities of metastasis from these models were used to calculate the area under the curve (AUC) with 95\% CI as measure of diagnostic performance. The AUC of the model incorporating both the new criteria and the size criterion was compared with the AUC of the model that incorporated only the size criterion. The difference in AUCs for the different models was tested using the method described by Hanley et al., which accounts for the fact that the AUCs are derived from the same sample of patients [25]. A p-value $<0.05$ was considered to be statistically significant. 
Table 1 Parameters of the head and neck MRI protocol

\begin{tabular}{|c|c|c|c|c|}
\hline \multirow[t]{2}{*}{ Parameters } & \multicolumn{4}{|l|}{ Sequences } \\
\hline & $\begin{array}{c}\text { TSE } \\
\text { T1-weighted }\end{array}$ & $\begin{array}{l}\text { SPIR }^{\mathrm{a}} \text { TSE } \\
\text { T2-weighted }\end{array}$ & $\begin{array}{c}\text { SPIR CE }{ }^{b} \\
\text { T1-weighted } \\
\end{array}$ & $\begin{array}{c}\text { SPIR CE } \\
\text { T1-weighted }\end{array}$ \\
\hline Field of view (mm) & 220 & 220 & 220 & 220 \\
\hline Slice orientation & Transverse & Transverse & Coronal & Transverse \\
\hline Matrix & 512 & 512 & 512 & 513 \\
\hline Section thickness (mm) & 3 & 3 & 3 & 3 \\
\hline No. of sections & 65 & 65 & 50 & 65 \\
\hline No. of signals acquired & 3 & 3 & 3 & 3 \\
\hline Acquisition time (min:s) & $5: 37$ & 5:09 & $6: 05$ & $6: 40$ \\
\hline TSE $^{\mathrm{c}}$ factor & 3 & 20 & 4 & 4 \\
\hline $\mathrm{TE}^{\mathrm{d}}(\mathrm{ms})$ & 13 & 80 & 14.50 & 14.50 \\
\hline $\operatorname{Tr}^{\mathrm{e}}(\mathrm{ms})$ & 530 & Shortest & 500 & 500 \\
\hline Slice gap & 0 & 0 & 0 & 0 \\
\hline
\end{tabular}

${ }^{\mathrm{a}}$ SPIR spectral pre saturation inversion recovery, ${ }^{\mathrm{b}} \mathrm{CE}$ contrast enhanced, ${ }^{\mathrm{c}} \mathrm{TSE}$ turbo spin echo,

${ }^{\mathrm{d}}$ TE echo time, ${ }^{\mathrm{e}} \mathrm{TR}$ repitition timeMR evaluation

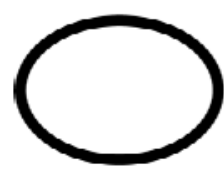

smooth

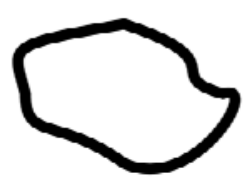

lobulated

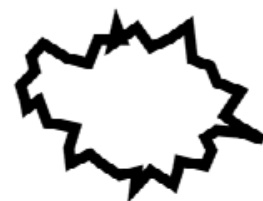

spiculated

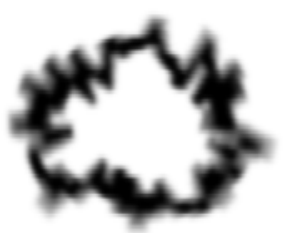

indistinct

Figure 1 Schematic drawing of the four borders for assessment of lymph nodes 

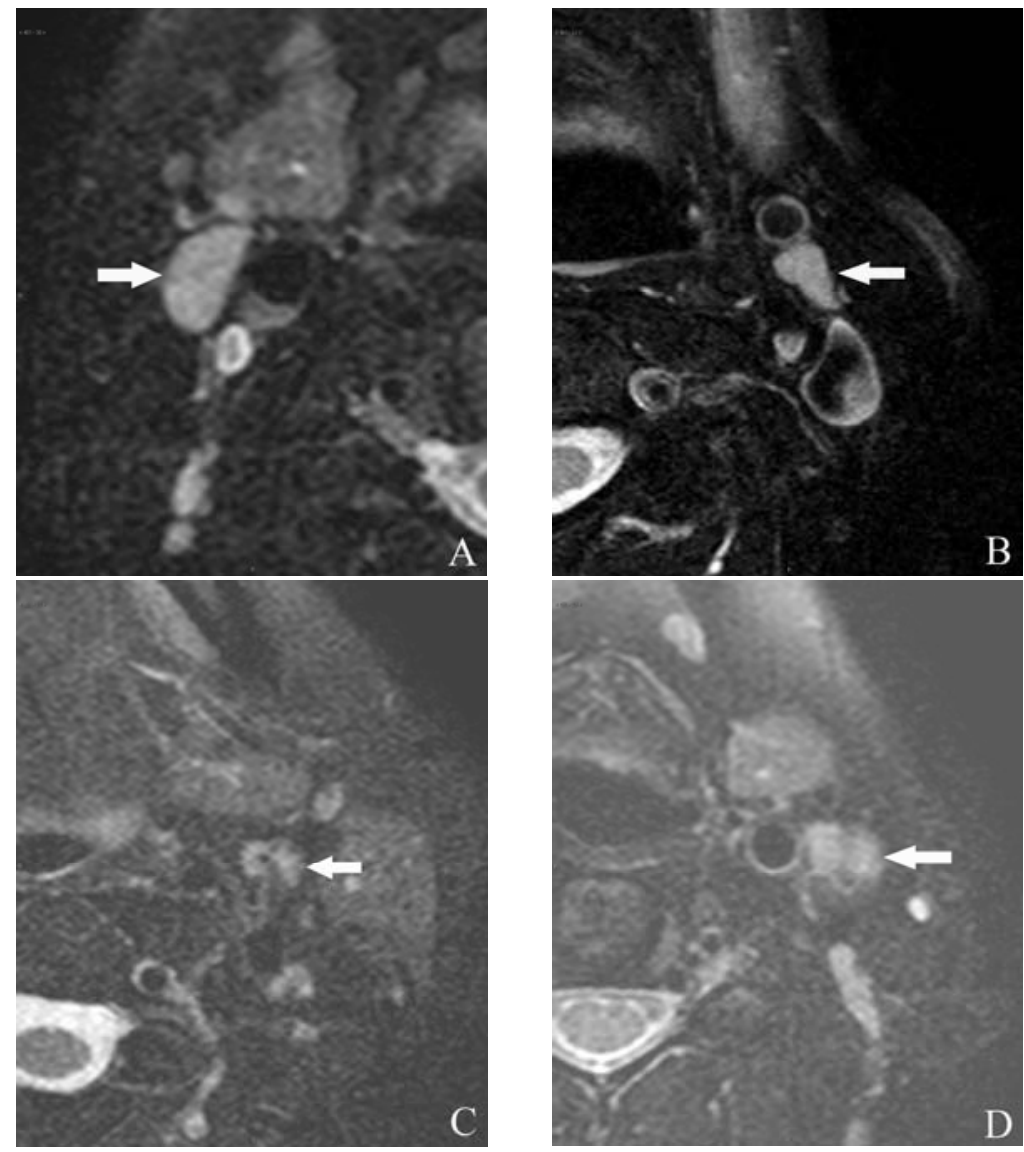

Figure 2 Transverse turbo spin-echo T2-weighted images of the neck. Examples of the four different scores on border regularity. The lymph nodes (arrows) show (A) a smooth border, (B) a lobulated border, (C) a spiculated border, and (D) an indistinct border

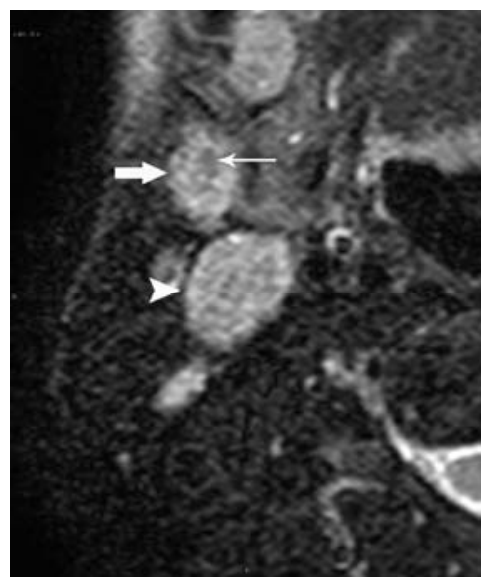

Figure 3 Transverse turbo spin-echo T2-weighted image of the neck showing an example of heterogeneous signal intensity (SI) in a lymph node in level II on the right side. The lymph node (arrowhead) with a homogenous SI, although a short axial diameter of $13 \mathrm{~mm}$, showed no metastasis, whereas the smaller lymph node (arrow) (short axial diameter $9 \mathrm{~mm}$ ) with a heterogeneous and eccentric area of low SI (small arrow) revealed metastasis at the pathologic examination 


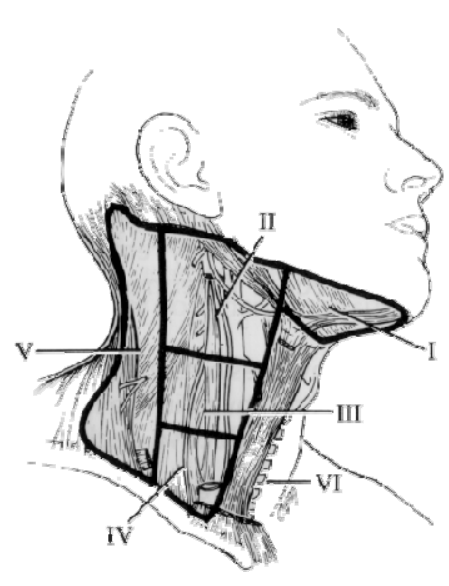

Figure 4 Schematic drawing of the different levels in the neck

\section{Results}

In 44 patients a neck dissection was performed: 9 supraomohyoid neck dissections and 37 radical (modified) neck dissections (in 2 patients a bilateral neck dissections). Distribution of the dissected neck levels was as follows; 32 level I, 41 level II, 34 level III, 25 level IV, and 8 level V.

At pathological examination a total of 261 lymph nodes was found: 71 lymph nodes with metastasis and 190 without metastasis (prevalence=27.2\%). In all levels together, a total of 111 lymph nodes was found in level II, 52 lymph nodes in level I, 65 lymph nodes in level III, 25 lymph nodes in level IV, and 8 lymph nodes in level V. Lymph node metastases were present in 33 patients (patient prevalence $=80.5 \%)$. The majority $(82 \%)$ of 261 assessed lymph nodes was scored as having a size $<10 \mathrm{~mm}$. The average pathologic yield per patient was 11.5 lymph nodes.

At assessment of the MR images of the dissected neck levels, a total of 360 lymph nodes was detected. Of these lymph nodes, 99 were shown to have a short axial diameter of $\leq 3 \mathrm{~mm}$. Because these lymph nodes were not detected at pathological examination, they were excluded from further analysis.

A total of 261 lymph nodes, which was found at pathological examination, was matched to lymph nodes that were detected on MRI by the two observers. 
For observer 1, scores were lacking in 1 lymph node metastasis, leaving 260 lymph nodes for analysis: 70 lymph nodes with metastasis and 190 without metastasis. For observer 2, complete data were available for all 261 lymph nodes.

Observer agreement was $\kappa=1.00$ for size, $\kappa=0.62$ for border irregularity, $\kappa=0.51$ for signal intensity on contrast-enhanced T1-weighted images, and $\kappa=0.51$ for signal intensity on T2-weighted images.

Table 2 shows the sensitivity, specificity, and diagnostic odds ratio (DOR) with $95 \%$ confidence interval $(95 \% \mathrm{CI})$ for the diagnosis of metastasis for the criteria border (abnormal versus normal) on T2-weighted images, signal intensity on contrast-enhanced T1-weighted images (heterogeneous versus homogeneous), and signal intensity on T2-weighted images (heterogeneous versus homogeneous) for the two observers. With respect to the new criteria, the highest DORs are found for border irregularity and signal intensity on T2-weighted images. The lowest DORs are found for the criterion contrast enhancement on T1-weighted images.

Table 3 shows the results for lymph nodes that were smaller than $10 \mathrm{~mm}$. The DORs associated with the new criteria are smaller compared to the DORs for all lymph nodes, but are still elevated, indicating that the morphologic criteria are also useful for the detection of metastases in small lymph nodes.

Table 4 shows the results from multivariate logistic regression analyses. The DORs associated with border irregularity were 2.61 (95\% CI: 1.12-6.08) and 66.2 (95\% CI: 20.4 -217) for observer 1 and 2, respectively; for heterogeneity on T2-weighted images DORs were 2.97 (95\% CI: 1.42-6.18) and 22.6 (95\% CI: 6.40-80.1). The DORs associated with heterogeneity on T1-weighted images are not significantly different from DOR=1.

Another observation was that for observer 2, the DOR associated with size was 1.02 , indicating that for this observer size had no additional diagnostic value when considered in combination with the other criteria.

The AUCs for predictive models using size only $(\leq 10 \mathrm{~mm}$ versus $>10 \mathrm{~mm})$ were 0.67 (95\% CI: $0.61-0.73$ ) and 0.68 (95\% CI: 0.62-0.74) for observer 1 and 2, respectively. After incorporation of the three new criteria into models, the AUCs increased significantly to 0.81 (95\% CI: $0.75-0.87)$ for observer 1 and to 0.96 (95\% CI: $0.94-0.98)$ for observer $2(p<0.001)$. When the criterion "homogeneity of the signal intensity on contrast-enhanced T1-weighted images" was omitted from the predictive model, there was only a very small decrease in AUC for 
observer 1 from 0.81 to 0.79 (95\% CI: $0.73-0.85$ ) and remained 0.96 (95\% CI: 0.93-0.98) for observer 2. This observation indicates that this criterion has little added diagnostic value.

Table 2 Sensitivity, specificity, and diagnostic odds ratio (DOR) with 95\% confidence interval (95\% CI) for the diagnosis of metastasis for the MR criteria: size, border irregularity, signal intensity (SI) on contrast-enhanced (CE) T1-weighted images and SI on T2-weighted images for all lymph nodes

\begin{tabular}{|c|c|c|c|c|c|c|}
\hline \multirow[b]{2}{*}{ All lymph nodes } & \multicolumn{3}{|l|}{ Observer 1} & \multicolumn{3}{|l|}{ Observer 2} \\
\hline & Sensitivity & Specificity & $\begin{array}{c}\text { DOR } \\
(95 \% \mathrm{CI})\end{array}$ & Sensitivity & Specificity & $\begin{array}{c}\text { DOR } \\
(95 \% \mathrm{CI})\end{array}$ \\
\hline $\begin{array}{l}\text { Short axial diameter }(\mathrm{mm}) \\
>10 \text { versus } \leq 10\end{array}$ & 43 & 92 & $\begin{array}{c}8.16 \\
(4.09-16.3)\end{array}$ & 42 & 92 & $\begin{array}{c}7.96 \\
(3.99-15.9)\end{array}$ \\
\hline $\begin{array}{l}\text { Borders on T2-weighted } \\
\text { images piculated/indistinct } \\
\text { versus smooth/lobulated }\end{array}$ & 63 & 84 & $\begin{array}{c}8.68 \\
(4.69-16.1)\end{array}$ & 87 & 94 & $\begin{array}{c}112 \\
(44.7-280)\end{array}$ \\
\hline $\begin{array}{l}\text { SI on CE T1-weighted } \\
\text { Images heterogeneous } \\
\text { versus homogeneous }\end{array}$ & 71 & 66 & $\begin{array}{c}4.81 \\
(2.65-8.71)\end{array}$ & 61 & 65 & $\begin{array}{c}2.89 \\
(1.65-5.05)\end{array}$ \\
\hline $\begin{array}{l}\text { SI on T2-weighted images } \\
\text { heterogeneous versus } \\
\text { homogeneous }\end{array}$ & 67 & 77 & $\begin{array}{c}6.99 \\
(3.83-12.7)\end{array}$ & 93 & 68 & $\begin{array}{c}28.6 \\
(11.3-72.3)\end{array}$ \\
\hline
\end{tabular}

Table 3 Sensitivity, specificity, and diagnostic odds ratio (DOR) with 95\% confidence interval (95\% CI) for the diagnosis of metastasis for the MR criteria: size, border irregularity, signal intensity (SI) on contrast-enhanced (CE) T1-weighted images and SI on T2-weighted images for lymph nodes $<10$ $\mathrm{mm}$ in short axial diameter

\begin{tabular}{|c|c|c|c|c|c|c|}
\hline \multirow[b]{2}{*}{ Lymph nodes $<10 \mathrm{~mm}$} & \multicolumn{3}{|c|}{ Observer 1} & \multicolumn{3}{|c|}{ Observer 2} \\
\hline & Sensitivity & Specificity & $\begin{array}{c}\text { DOR } \\
(95 \% \mathrm{CI})\end{array}$ & Sensitivity & Specificity & $\begin{array}{c}\text { DOR } \\
(95 \% \mathrm{CI})\end{array}$ \\
\hline $\begin{array}{l}\text { Borders on T2-weighted } \\
\text { images piculated/indistinct } \\
\text { versus smooth/lobulated }\end{array}$ & 40 & 87 & $\begin{array}{c}4.61 \\
(2.12-9.99)\end{array}$ & 78 & 97 & $\begin{array}{c}99.6 \\
(33.1-299)\end{array}$ \\
\hline $\begin{array}{l}\text { SI on CE T1-weighted } \\
\text { images heterogeneous } \\
\text { versus homogeneous }\end{array}$ & 53 & 70 & $\begin{array}{c}2.52 \\
(1.25-5.08)\end{array}$ & 42 & 68 & $\begin{array}{c}1.49 \\
(0.74-3.00)\end{array}$ \\
\hline $\begin{array}{l}\text { SI on T2-weighted images } \\
\text { heterogeneous versus } \\
\text { homogeneous }\end{array}$ & 53 & 79 & $\begin{array}{c}4.24 \\
(2.06-8.71)\end{array}$ & 88 & 68 & $\begin{array}{c}15.6 \\
(5.70-41.8)\end{array}$ \\
\hline
\end{tabular}


Table 4 Results of multivariate logistic regression analyses. Regression coefficients $(\beta)$, diagnostic odds ratios (DOR) with 95\% confidence intervals (95\% CI) for the size criterion, and the three new assessed MR criteria border, signal intensity (SI) on contrast-enhanced (CE) T1-weighted images and T2-weighted images for both MR observers

\begin{tabular}{lcccc}
\hline & \multicolumn{2}{c}{ Observer 1 } & \multicolumn{2}{c}{ Observer 2 } \\
\cline { 2 - 5 } MRI criteria & $\beta$ & DOR $(95 \% \mathrm{CI})$ & $\beta$ & DOR (95\% CI) \\
\hline Size $>10$ versus $\leq 10 \mathrm{~mm}$ & 1.06 & $2.89(1.25-6.70)$ & 0.02 & $1.02(0.25-4.18)$ \\
$\begin{array}{l}\text { Border on T2-weighted images } \\
\text { versus smooth/lobulated }\end{array}$ & 0.96 & $2.61(1.12-6.08)$ & 4.20 & $66.2(20.4-217)$ \\
$\begin{array}{l}\text { SI on CE T1-weighted images } \\
\text { versus homogeneous }\end{array}$ & 0.37 & $1.45(0.67-3.14)$ & 0.68 & $1.97(0.70-5.59)$ \\
$\begin{array}{l}\text { SI on T2-weighted images } \\
\text { versus homogeneous }\end{array}$ & 1.09 & $2.97(1.42-6.18)$ & 3.12 & $22.6(6.40-80.1)$ \\
\hline
\end{tabular}




\section{Discussion}

The principal finding of this study was that in addition to the size criterion, new criteria, such as border irregularity and heterogeneity on T2-weighted images, result in a better diagnostic performance of MRI for the detection of cervical lymph node metastases in HNSCC.

Accurate assessment of cervical lymph node metastases in HNSCC is important regarding prognosis and choice of management. Detection of lymph node metastases is usually based on the short axial diameter as size criterion.

However, many studies have shown that the size criterion alone was not accurate enough as predictor for lymph node metastasis [16]. Sensitivity is poor when the commonly chosen cutoff value of $10 \mathrm{~mm}$ is used, and although use of a lower cutoff value, as proposed by van den Brekel et al., results in higher sensitivity, lower cutoff values are associated with low specificity [26]. We evaluated in this study the effect on sensitivity and specificity when lowering the cutoff point from 10 to 7 $\mathrm{mm}$ in short axial diameter; sensitivity raised from $43 \%$ to $79 \%$, but the associated specificity expectedly lowered from $92 \%$ to $64 \%$. However, the corresponding diagnostic odds ratios (DORs) did not differ significantly (DOR=8.16 with $95 \%$ CI $4.09-16.3$ versus DOR=6.43 with 95\% CI 3.38-12.23).

Another assessment of lymph node dimensions is the measurement of ratios of maximum longitudinal to maximum short axial diameter ( $1 / \mathrm{s}$ ratio). A ratio less than 2, like in round lymph nodes versus elliptical lymph nodes, is indicative of metastatic disease, but this is related to enlarged lymph nodes. Because in our presented series $82 \%$ of all assessed lymph nodes were not enlarged - showing short axial diameters less than $10 \mathrm{~mm}$ - we used in this study the $10-\mathrm{mm}$ short axial diameter as cutoff point.

Additional morphological criteria for the detection of lymph node metastasis have been evaluated in this study: the degree of border irregularity on T2-weighted images, homogeneity of the signal intensity on contrast-enhanced T1-weighted images, and the homogeneity of the signal intensity on T2-weighted images of lymph nodes.

The results of univariate analyses, with which we compared the diagnostic performance of all four criteria under study, indicate that all criteria, when used alone, help to discriminate between lymph nodes with and without metastasis, but that the discriminative ability was lowest for degree of homogeneity of signal 
intensity on contrast-enhanced T1-weighted images. This finding also holds after restricting the analysis to lymph nodes smaller than $10 \mathrm{~mm}$. However, when compared with the analysis involving all lymph nodes, DORs were lower, indicating that within small nodes the discriminative ability is poorer. An exception was border irregularity, which for observer 2 was still associated with a high DOR of about 100 (compared with a DOR of 112 in the analysis based on all lymph nodes).

Another morphological criterion that has been used is the presence of central necrosis. However, central necrosis is often seen in the larger involved lymph nodes, which based on their size only would have been classified as malignant anyhow [17-20]. The majority of lymph nodes in our series (82\%) showed subcentrimetrical short axial diameters.

Curtin et al. have already described the diagnostic value of the appearance of internal abnormalities in lymph nodes on CT and MRI; however, the performance of MRI was not changed significantly by the addition of information on internal abnormalities [18].

Evaluation of the results of multivariate logistic regression analysis indicates that, if used in combination with the size criterion, for both observers the scores on border irregularity and heterogeneous signal intensity on T2-weighted images contribute significantly to the prediction of the presence of metastatic lymph nodes, whereas the criterion heterogeneity of signal intensity on contrast-enhanced T1-weighted images has no additional diagnostic value.

Explanations for the MR features in metastatic lymph nodes could be the following. Firstly, changes of a smooth or lobulated border of normal lymph nodes into a spiculated or indistinct border could be due to direct extra-nodal tumor infiltration into the peri-nodal fatty tissue. Secondly, this phenomenon could be explained by a desmoplastic reaction around the affected lymph node, the similar feature that can be observed around a primary tumor site. Thirdly, tumor infiltration and presence of softening or necrosis within lymph nodes usually show on the pathological examination an irregular and heterogeneous pattern. This architectural distortion of the nodal parenchyma results in an irregular signal intensity on T2-weighted images and in a heterogeneous signal intensity on the contrast-enhanced T1-weighted images.

The discrepancies in diagnostic performance between the two observers and low inter-observer agreement regarding the new morphological criteria could be 
explained by the fact that observer 2, experienced in head and neck radiology, was better aware of the diagnostic value of border irregularity and homogeneity of signal intensity than his colleague who had less experience in the assessment of lymph node involvement. In this respect, the results also show that the criterion size for lymph nodes has limited discriminative value for the experienced observer 2. For the more experienced observer, border irregularity and heterogeneity of signal intensity on T2-weighted images are more decisive in the diagnosis. This is reflected by the results of the univariate analysis with high DORs for the criteria border and signal intensity on T2-weighted images (112 and 28.6, respectively), when compared with the low DOR for the size criterion (7.96). Also, in the multivariate logistic regression analysis high DORs are presented for those criteria (66.2 and 22.6, respectively). This is in contrast to the DOR of 1.02 for the size criterion.

\section{Conclusions}

Newly assessed morphological criteria like border irregularity and heterogeneity of signal intensity on T2-weighted images in addition to the size criterion significantly improved the detection of cervical lymph node metastases on MRI in patients with HNSCC. Experience in assessment of cervical lymph nodes is required to adequately use these morphological criteria. 


\section{References}

1. Kalnins IK, Leonard AG, Sako K et al. Correlation between prognosis and degree of lymph node involvement in carcinoma of the oral cavity. Am J Surg 1977;134:450-454

2. Jakobsen J, Hansen $\mathrm{O}$, Jorgensen $\mathrm{KE}$ et al. Lymph node metastases from laryngeal and pharyngeal carcinomas-calculation of burden of metastasis and its impact on prognosis. Acta Oncol 1998;37:489-493

3. Kehrl W, Wenzel S, Niendorf A. [Effect of various forms of metastatic lymph node involvement on prognosis of squamous epithelial carcinomas of the upper aerodigestive tract]. Laryngorhinootologie 1998;77:569-575

4. Ganzer U, Meyer-Breiting E, Ebbers J et al. [Effect of tumor size on lymph node metastasis and type of treatment on the prognosis of hypopharyngeal cancer]. Laryngol Rhinol Otol (Stuttg) 1982;61:622-628

5. Leemans CR, Tiwari RM, van der Waal I et al. [Neck lymph node dissection in squamous cell carcinoma originating in the head-and-neck area; the significance for the prognosis]. Ned Tijdschr Geneeskd 1992;36:221-225

6. Foote RL, Olsen KD, Davis DL. Base of tongue carcinoma: patterns of failure and predictors of recurrence after surgery alone. Head Neck 1993;15:300-307

7. Leemans CR, Tiwari R, Nauta JJ et al. Recurrence at the primary site in head and neck cancer and the significance of neck lymph node metastases as a prognostic factor. Cancer 1994;73:187-190

8. Taylor JM, Mendenhall WM, Lavey RS. Time-dose factors in positive neck nodes treated with irradiation only. Radiother Oncol 1991;22:167-173

9. Newman JP, Terris DJ, Pinto HA et al. Surgical morbidity of neck dissection after chemoradiotherapy in advanced head and neck cancer. Ann Otol Rhinol Laryngol 1997; 106:117-122

10. Hillel A, Patten C. Neck dissection: morbidity and rehabilitation. Cancer Treat Res 1990;52:133-147

11. Sanguineti G, Sormani MP, Benasso M et al. Late local treatment morbidity after accelerated radiotherapy or alternating chemoradiotherapy for advanced head and neck carcinoma. Tumori 2002;88:313-320

12. Bentzen SM, Saunders MI, Dische $S$ et al. Radiotherapy-related early morbidity in head and neck cancer: quantitative clinical radiobiology as deduced from the CHART trial. Radiother Oncol 2001;60:123-135

13. Ferlito A, Rinaldo A, Robbins KT et al. Changing concepts in the surgical management of the cervical node metastasis. Oral Oncol 2003;39:429-435

14. Olsen KD, Caruso M, Foote RL. Primary head and neck cancer. Histopathologic predictors of recurrence after neck dissection in patients with lump node involvement. Arch Otolaryngol Head Neck Surg 1994;120:1370-1374

15. Byers RM, Wolf PF, Ballantyne AJ. Rationale for elective modified neck dissection. Head Neck Surg 1988;10:160-167

16. de Bondt RB, Nelemans PJ, Hofman PA et al. Detection of lymph node metastases in head and neck cancer: A meta-analysis comparing US, USgFNAC, CT and MR imaging. Eur J Radiol 2007

17. van den Brekel MW, Castelijns JA, Stel HV et al. Modern imaging techniques and ultrasoundguided aspiration cytology for the assessment of neck node metastases: a prospective comparative study. Eur Arch Otorhinolaryngol 1993;250:11-17

18. Curtin HD, Ishwaran H, Mancuso AA et al. Comparison of CT and MR imaging in staging of neck metastases. Radiology 1998;207:123-130 
19. Braams JW, Pruim J, Freling NJ et al. Detection of lymph node metastases of squamous-cell cancer of the head and neck with FDG-PET and MRI. J Nucl Med 1995;36:211-216

20. Feinmesser R, Freeman JL, Noyek AM et al. MRI and neck metastases: a clinical, radiological, pathological correlative study. J Otolaryngol 1990;19:136-140

21. Sigal R, Vogl T, Casselman J et al. Lymph node metastases from head and neck squamous cell carcinoma: MR imaging with ultrasmall superparamagnetic iron oxide particles (Sinerem MR) - results of a phase-III multicenter clinical trial. Eur Radiol 2002;12:1104-1113

22. Kim JH, Beets GL, Kim MJ et al. High-resolution MR imaging for nodal staging in rectal cancer: are there any criteria in addition to the size? Eur J Radiol 2004;52:78-83

23. Fleming I, Cooper J, Henson D. AJCC Staging manual. Lippenscott-Raven 5th ed, Philadelphia 1997

24. Glas AS, Lijmer JG, Prins $\mathrm{MH}$ et al. The diagnostic odds ratio: a single indicator of test performance. J Clin Epidemiol 2003;56:1129-1135

25. Hanley JA, McNeil BJ. A method of comparing the areas under receiver operating characteristic curves derived from the same cases. Radiology 1983;148:839-843

26. van den Brekel MW, Castelijns JA, Snow GB. The size of lymph nodes in the neck on sonograms as a radiologic criterion for metastasis: how reliable is it? AJNR Am J Neuroradiol 1998;19:695-700 
$\longrightarrow$ 


\title{
CHAPTER 6
}

\section{Diagnostic accuracy and additional value of diffusion weighted imaging for discrimination of malignant cervical lymph nodes in head and neck squamous cell carcinoma.}

\author{
R.B.J. de Bondt \\ M.C. Hoeberigs \\ P.J. Nelemans \\ W.M.L.L.G. Deserno \\ C. Peutz-Kootstra \\ B. Kremer \\ R.G.H. Beets-Tan
}

Neuroradiology 2009 Mar; 51(3): 183-92

Awarded with the annual first prize of the European Society of Head and Neck Radiology (ESHNR) 2008 


\section{Abstract}

Introduction: The aim was to determine the diagnostic accuracy and additional value of diffusion-weighted imaging (DWI) for detection of malignant lymph nodes in head and neck squamous cell carcinoma (HNSCC).

Methods: 219 lymph nodes, predominantly smaller than $10 \mathrm{~mm}$ (95.4\%), in 16 consecutive patients were evaluated at 1.5 Tesla. Lymph nodes were evaluated for maximum short axial diameter, morphological criteria and apparent diffusion coefficient (ADC) values $\left(b=0\right.$ and $\left.b=1000 \mathrm{sec} / \mathrm{mm}^{2}\right)$. Sensitivity, specificity, positive and negative predictive values as well as diagnostic odds ratios (DORs) and areas under the curves (AUCs) of ROC curves, were calculated for the various MRI criteria individually and in combination. Histological examination of lymph nodes in the neck dissection specimen was the gold standard to determine malignant involvement.

Results: The optimal ADC threshold was $1.0 \times 10^{-3} \mathrm{~mm}^{2} / \mathrm{sec}$. Using this cut-off point, sensitivity and specificity were $92.3 \%$ and $83.9 \%$, respectively. When used in combination with size and morphological criteria, ADC value $<1.0 \times 10^{-3}$ $\mathrm{mm}^{2} / \mathrm{sec}$ was the strongest predictor of presence of metastasis (DOR=97.6). A model which added ADC values to the other MRI criteria performed significantly better than a model without $\mathrm{ADC}$ values: $\mathrm{AUC}=0.98$ versus $\mathrm{AUC}=0.91$ $(p=0.036)$.

Conclusion In this study, with predominantly small lymph nodes, the ADC criterion is the strongest independent predictor of presence of metastasis. The use of ADC values in combination with the other MRI criteria significantly improves the discrimination between malignant and benign lymph nodes. 


\section{Introduction}

The presence of cervical lymph node metastases is an important prognostic factor in patients with head and neck squamous cell carcinoma (HNSCC) as this significantly worsens the treatment outcome [27-34]. Radiological imaging plays an increasingly important role in accurate assessment of the lymph node status of the neck.

Frequently used criteria for differentiation between benign and malignant lymph nodes are the presence of necrosis - mainly seen in larger lymph nodes - and assessment of lymph node morphology such as borders and internal heterogeneity $[11,35]$. Also the introduction of lymph node specific contrast agents like ultra small particles iron oxide (USPIO) on MRI provides differentiation between benign and malignant lymph nodes in the head and neck and results are improving [36-41].

Another commonly used criterion is the maximum short axial diameter for which several studies have been undertaken to determine the optimal cut off size [42, 43]. Small lymph nodes with a maximum short axial diameter below $10 \mathrm{~mm}$ are more challenging for radiologists, because the mere use of this size criterion will result in misclassification of malignant lymph nodes as normal on MRI evaluation. According to the literature, the performance of MRI is still poor in the assessment of lymph node metastases, and this concerns especially the detection of metastases in small lymph nodes [36].

Diffusion weighted imaging (DWI) is a non-invasive magnetic resonance imaging (MRI) technique that provides image contrast dependent on the molecular motion of water. Any architectural changes in the proportion of extra cellular to intra cellular water protons - like metastases in lymph nodes - will alter the diffusion coefficient of the tissue. Therefore, DWI provides in characterization of different tissues and lesions. Previous reports showed the utility of DWI in the region of the head and neck for different pathology[44-51]. In studies focused on nodal staging, the imaging results were based on series with only clinical positive necks in which large lymph nodes were selected for evaluation [52-55]. Studies focusing on mainly negative necks at clinical examination are scarce. 
The aim of this study was to prospectively determine the diagnostic accuracy and additional value of DWI-MRI to conventional MRI - using size and morphology criteria - for differentiation between malignant and normal cervical lymph nodes.

\section{Patients and Methods}

\section{Patients}

The local medical ethics committee approved this prospective study. Between March 2006 and December 2007 a series of 16 consecutive patients with a HNSCC who were scheduled for a supraomohyoid neck dissection (SOHND), selective or radical neck dissection (RND) enrolled in this study. All patients underwent MR imaging as part of the routine diagnostic work up, the findings of diffusion weighted imaging (DWI) were not used for clinical decision making. MR imaging was performed previous to ultrasonography with fine needle aspiration cytology of lymph nodes in the neck.

\section{MR imaging}

All examinations were performed at 1.5 T (Gyroscan, Powertrack 6000, Philips, Best, The Netherlands) using a Head-Neck coil (Philips, Best, The Netherlands). The Synergy Head-Neck coil was used for both the conventional and diffusion weighted MR imaging, the range was from the base of skull to the level of the clavicles. To ascertain correlation of the conventional images and DWI, all sequences were acquired with similar geometry.

The conventional MR protocol consisted of axial fat suppressed T2-weighted (TR/TE 1704/80), axial and coronal T1-weighted (TR/TE 400/12), contrast enhanced axial and coronal fat suppressed T1-weighted (TR/TE 740/12) and coronal STIR (TR/TE/TI 5868/60/15) sequences. Slice thickness was $3 \mathrm{~mm}$, there was no inter-slice gap. The matrix was 272 x 512 with a field of view 220 (RFOV $80 \%)$.

Diffusion weighted images were acquired in the axial plane by using a gradient single-shot echo planar imaging (EPI) sequence. The sequence was repeated for two b-values $b=0$ and $b=1000 \mathrm{~s} / \mathrm{mm}^{2}$ with the following parameters: TR/TE $5666 / 70$, section thickness was $3 \mathrm{~mm}$ according to the conventional protocol, the matrix was $272 \times 512$ with a field of view 400 (RFOV 80\%), there was no interslice gap, the number of signal acquisition (NSA) was 6 . A total of 64 slices were acquired which covered the whole neck area from the skull base to the clavicles. 
The total scan time was 4 min $54 \mathrm{sec}$. DWI was performed before the contrast enhanced T1-weighted sequences. Apparent diffusion coefficient (ADC) maps were automatically constructed by the post processing software of the MR system.

\section{Image evaluation}

Two radiologists with specific experience in head and neck imaging (12 and 4 years, respectively), and taking part of multidisciplinary head and neck oncology conferences in a referral hospital, independently evaluated the conventional images. Both observers were blinded to each other's MR assessments and clinical information.

On the conventional MR images, lymph nodes were assessed for size and morphological criteria. All lymph nodes were determined by position and slice number per neck level, the maximum short axial diameter was recorded per lymph node and lymph nodes were classified as normal $(\leq 10 \mathrm{~mm})$ or malignant $(>10$ $\mathrm{mm}$ ). Furthermore, for each lymph node it was predicted whether it was benign or malignant by using the following morphological criteria determined on the fat suppressed T2-weighted images: the border of the lymph node (smooth, lobulated, spiculated or indistinct). Figure 1: presence of smooth and lobulated borders were considered as indicative of normal lymph nodes whereas that of spiculated and indistinct borders of malignant lymph nodes. Another criterion was the degree of homogeneity of the signal intensity; homogeneous signal intensity was considered as normal whereas heterogeneous signal intensity was considered as indicative of malignant lymph nodes.

Diffusion weighted images were evaluated on the MRI scanner workstation on which the ADC maps were constructed automatically by the post processing software of the MR system. In consensus the two radiologists, who also assessed the conventional MR images, performed the evaluation of the diffusion weighted images. To ascertain a node-by-node correlation between the conventional images versus the diffusion weighted images, all lymph nodes on the T1-weighted and T2-weighted images were correlated to the corresponding b1000 images. On these b1000 images a region of interest (ROI) was drawn manually around each single lymph node, the ROI was copied to the ADC map and the ADC value was automatically calculated by the post processing software of the MR machine. This was done on all subsequent sections that contained lymph node parenchyma to ensure that most of the lymph node was included. The final ADC value of a lymph node was an average of all values from one single lymph node; this was expressed as mean \pm standard deviation. Figure 2 demonstrates an example of this post processing technique. A necrotic area in a lymph node, determined as a hyper- 
intense region on the T2-weighted image with a hypo-intense region on the (contrast enhanced) T1-weighted images, was excluded from the area of analysis.

\section{Pathological examination}

All neck dissections were performed "en bloc". Immediately after the resection the surgeon positioned the neck dissection specimen on a schematic drawing of the neck in real proportions and fixed it with needles. The pathologist manually identified and localized the lymph nodes per neck level in the specimen. The maximum short axial diameter of all lymph nodes was recorded. Subsequently, lymph nodes were fixed, sectioned and hematoxyline-eosine (HE) stained and the presence of tumor in each lymph node was examined microscopically. The results at pathology were used as the reference standard.

\section{Pathological-radiological correlation}

The neck was subdivided according to the six different levels in agreement with the classification of the American Joint Committee on Cancer (AJCC); this classification was used by the radiologists when viewing the MR images and used by the pathologist when interpreting the specimen after neck dissection [56].

The results of the measurements on MRI were compared with the results of the pathological examination of the neck dissection specimens. By recording the combination of the maximum short axial diameter and the exact location of each lymph node per neck level - related to surrounding anatomic structures such as blood vessels, muscles and salivary glands - it was possible to perform a topographic correlation for each lymph node between the pathological examination and the MR images.

\section{Statistical analysis}

Inter observer agreement with respect to the evaluation of the criteria size (maximum short axial diameter), border irregularity and homogeneity of signal intensity on T2-weighted images was expressed by Cohen's kappa-coefficient $(\kappa)$.

For each lymph node, the scores on MRI criteria were correlated with the results of pathologic examination of the neck dissection specimen. Pathologic results with respect to presence or absence of lymph node metastasis were used as standard of reference. Results on MRI criteria were dichotomized and diagnostic accuracy was evaluated in terms of sensitivity, specificity, positive predictive value (PPV) and negative predictive value (NPV). 
Furthermore, discriminative ability was expressed by the diagnostic odds ratio (DOR). The higher the DOR, the higher the ability of a particular criterion to discriminate between lymph nodes with and without metastasis. A DOR=1 indicates that the criterion under study has no diagnostic value; a $\mathrm{DOR}>1$ indicates that the criterion has ability to discriminate between lymph nodes with and without metastasis.

The independent contribution of each criterion under study was analyzed using multivariate logistic regression models. In these models, presence or absence of metastasis according to pathologic examination was the dependent variable and the dichotomized results on MRI criteria were entered as independent variables. Diagnostic odds ratios (DORs), which can be derived from these models by exponentiation of the regression coefficients, indicate to what extent each criterion in the model can be used as an independent predictor of the presence of metastasis in a lymph node.

Finally, the added diagnostic value of ADC values to the other MRI criteria (size, border irregularity and signal intensity on T2-weighted images) was analyzed by using multivariate logistic regression models, which included different sets of MRI criteria. Model-derived predicted probabilities were used to construct receiver operating characteristic (ROC) curves. Areas under the curve (AUCs) with 95\% confidence intervals were used to compare the diagnostic accuracy of various models. Differences between the AUCs were tested for statistical significance using the method described by Hanley et al., which accounts for the fact that the AUCs are derived from the same sample of patients. Statistical analyses were performed with SPSS 13 and STATA. In all analyses, p-values $<0.05$ were considered as indicator for statistical significance[57].

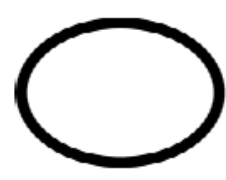

smooth

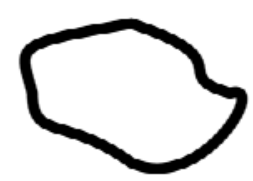

lobulated

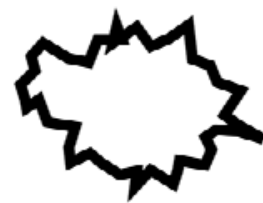

spiculated

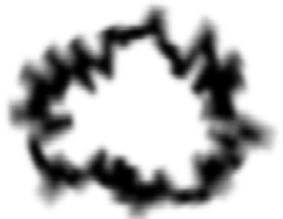

indistinct

Figure 1 Schematic drawing of the 4 borders for assessment of cervical lymph nodes. 


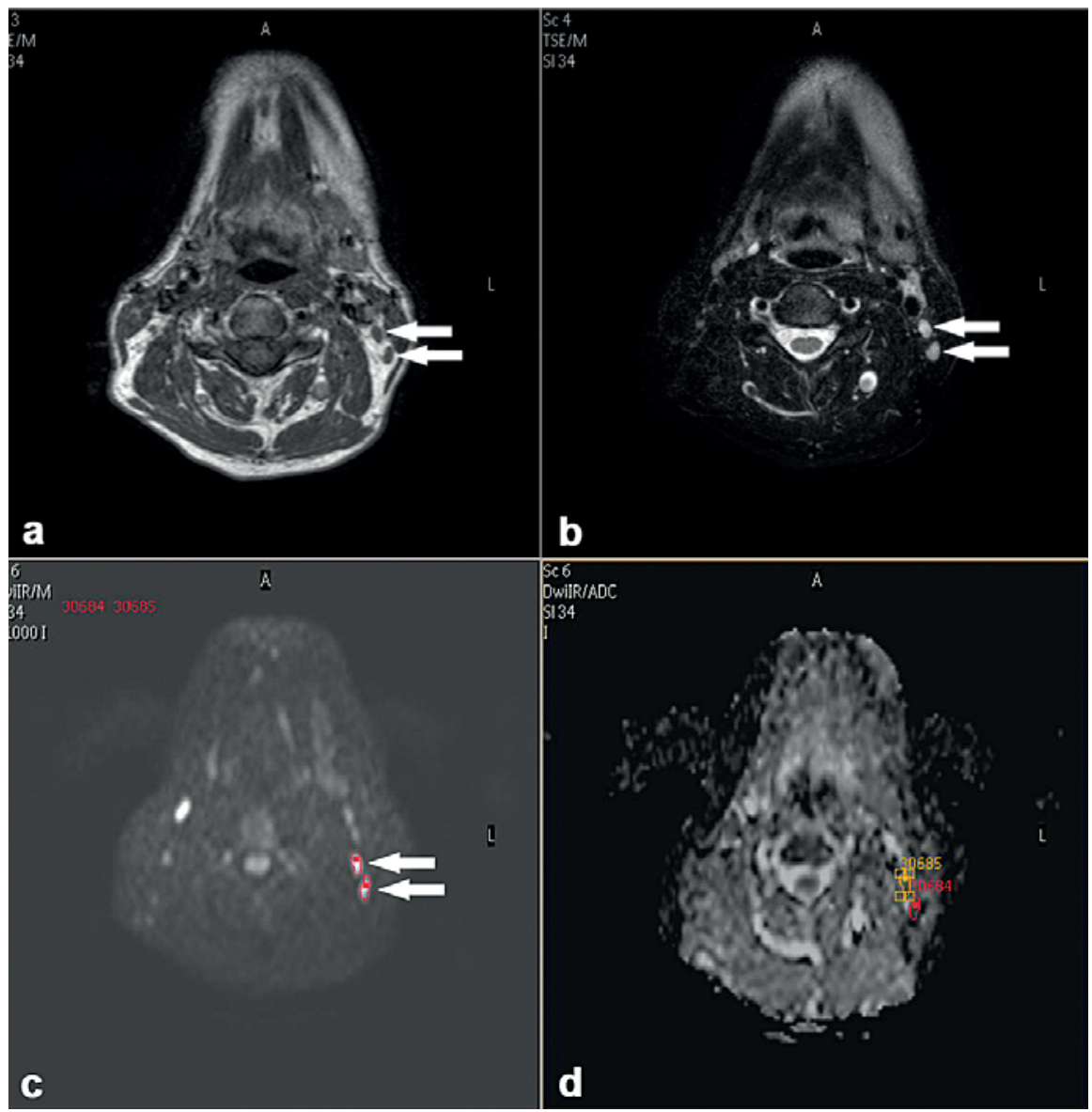

Figure 2 Overview of measurement of the apparent diffusion coefficient (ADC) value of cervical lymph nodes. Axial T1-weighted (a) and fat suppressed T2-weighted (b) images are used to ascertain matching between lymph nodes (arrows) on the conventional and the diffusion weighted imaging (DWI). Two small lymph nodes (arrows) can also be observed on the DWI b1000 image (c), on this image regions of interest are drawn around the lymph nodes and automatically copied to the ADC map (d) on which reference numbers are correlated to the ADC values.

\section{Results}

In 16 patients (mean age 59 years [range 40-77 years]; 7 women and 9 men) a total of 17 neck dissections were performed; supraomohyoid neck dissection (SOHND) $n=4$, radical neck dissection (RND) $n=4$ and selective neck dissection $n=9$. Distribution of the total of 58 dissected neck levels was as follows; level I $n=17$, level II $n=17$, level III $n=12$, level IV $n=5$ and level V $n=7$. 
Relevant patient characteristics, clinical findings, TNM classification and details of the performed neck dissections are summarized in Table 1.

At assessment of the MR images of the 58 dissected neck levels a total of 281 lymph nodes were detected. Of these lymph nodes, 62 had a short axial diameter of $\leq 2 \mathrm{~mm}$. Because these lymph nodes were not detected at the pathological examination they were excluded from further analysis.

The large majority (13 out of 16) of patients presented without palpable lymph nodes at clinical examination (clinical negative neck), only a few lymph nodes exceeded the maximum short axial diameter of $10 \mathrm{~mm}$ at pathological and radiological examination.

Pathological examination revealed 219 lymph nodes, which were matched to the lymph nodes on the MR images. Most lymph nodes were situated at levels I $(n=45)$, level II $(n=100)$ or level III $(n=49)$. Only 25 nodes were detected at level IV $(n=7)$ or level V $(n=18)$. Evidence of metastases was demonstrated in 26 lymph nodes (node-based prevalence $=11.8 \%$ ) and in 11 of 16 patients (patient-based prevalence $=68.8 \%)$. Lymph node size in relation to the presence of metastases ( $n=26)$ was as follows: $5 \mathrm{~mm} n=5 ; 6 \mathrm{~mm} n=4 ; 7 \mathrm{~mm} n=3 ; 8 \mathrm{~mm} n=6 ; 9 \mathrm{~m} n=1$; $10 \mathrm{~mm} n=3 ; 15 \mathrm{~mm} n=1 ; 18 \mathrm{~mm} n=1 ; 20 \mathrm{~mm} n=1 ; 30 \mathrm{~mm} n=1$. The majority (95.4\%) of lymph nodes on MRI showed a maximum short axial diameter $\leq 10$ $\mathrm{mm}$. Only 10 nodes $(4.6 \%)$ were $>10 \mathrm{~mm}$. Of these 10 pathological determined lymph nodes, 6 were negative at pathological examination (range $11-12 \mathrm{~mm}$ in maximum short axial diameter). Four lymph nodes, with maximum short axial diameter ranging from $15-30 \mathrm{~mm}$, showed massive metastasis. Twenty-two of the 26 metastases were found in the lymph nodes $\leq 10 \mathrm{~mm}$. Figure 3 illustrates a lymph node with maximum short axial diameter of $12 \mathrm{~mm}$ with an ADC value of $1.3 \mathrm{x}$ $10^{-3} \mathrm{~mm}^{2} / \mathrm{sec}$, pathological examination revealed no metastasis.

Observer agreement for the three morphological criteria was $\kappa=0.86$ for size, $\kappa=$ 1.00 for border irregularity and $\kappa=0.91$ for signal intensity on the T2-weighted images.

Table 2 presents combinations of sensitivity and specificity that were found using different cut-off values for ADC. Lower ADC values correspond with higher probability of lymph node metastasis. ADC values of malignant lymph nodes were significantly lower compared with benign lymph nodes with mean (standard deviation=SD) values of $0.85 \times 10^{-3} \mathrm{~mm}^{2} / \mathrm{sec}\left(\mathrm{SD} 0.19 \times 10^{-3} \mathrm{~mm}^{2} / \mathrm{sec}\right)$ and $1.2 \times$ $10^{-3} \mathrm{~mm}^{2} / \mathrm{sec}\left(\mathrm{SD} 0.24 \times 10^{-3} \mathrm{~mm}^{2} / \mathrm{sec}\right.$ ), respectively. 
High sensitivity combined with acceptable specificity was found for a cut-off point of $<1.0 \times 10^{-3} \mathrm{~mm}^{2} / \mathrm{sec}$ versus $>1.0 \times 10^{-3} \mathrm{~mm}^{2} / \mathrm{sec}$. This cut-off value was used for further analyses.

Table 3 shows the sensitivity, specificity, positive predictive value (PPV) and negative predictive value (NPV) for dichotomized ADC values and the other MRI criteria such as size, border irregularity and signal intensity on T2 weighted images. Both the dichotomized size and ADC criteria are associated with the highest sensitivity, but the high sensitivity for size is compromised by a high proportion of false positive results.

Table 4 shows diagnostic odds ratios (DORs) with 95\% confidence intervals (95\% CI) for the individual MRI criteria when they are used separately (derived from univariate analyses) and for the individual MRI criteria when they are used in combination with each other (derived from multivariate analyses). When used separately, the border criterion is associated with the highest ability to discriminate between malignant and benign lymph nodes ( $D O R=90.1)$. However, when used in combination, the ADC criterion is the strongest independent predictor of presence of metastasis (DOR=97.6) followed by the border criterion ( $\mathrm{DOR}=56.0)$. When evaluated in combination with the other criteria, the DOR of 4.4 (with $95 \% \mathrm{CI}$ : 0.7-28.0) associated with size no longer differs significantly from 1 and thus has no independent predictive value.

Table 5 presents areas under the curve (AUCs) with 95\% confidence intervals from various models. Model 3, adding ADC values to the other MRI criteria (size, border irregularity and signal intensity on T2-weighted images) performs significantly better than model 2 that does not add $\mathrm{ADC}$ values to the other three criteria: $\mathrm{AUC}=0.98$ versus $\mathrm{AUC}=0.91(p=0.036)$. Moreover, model 1, which includes only $\mathrm{ADC}$ as predictor of presence of metastasis, performs nearly as well as model 2, which includes the other three MRI criteria as predictors: $A U C=0.89$ versus $\mathrm{AUC}=0.91(p=0.52)$ 
Table 1 Clinical findings in 16 consecutive patients with primary head and neck squamous cell carcinoma, clinical TNM classification and details of the performed neck dissections

\begin{tabular}{|c|c|c|c|c|c|c|c|c|}
\hline \multirow{2}{*}{$\begin{array}{l}\text { Patient } \\
\text { no. }\end{array}$} & \multirow{2}{*}{$\begin{array}{c}\text { Age } \\
\text { (years) }\end{array}$} & \multirow[t]{2}{*}{ Gender } & \multicolumn{2}{|l|}{ Clinical presentation } & \multirow{2}{*}{$\begin{array}{c}\text { Clinical } \\
\text { TNM }\end{array}$} & \multicolumn{2}{|l|}{ Neck dissection (ND) } & \multirow{2}{*}{$\begin{array}{c}\text { Dissected } \\
\text { levels }\end{array}$} \\
\hline & & & Primary tumor location & Side & & Type & Side & \\
\hline 1 & 64 & M & Tongue & $\mathrm{R}$ & T2N0M0 & Selective ND & $\mathrm{R}$ & I-III,V \\
\hline 2 & 75 & M & Nasopharynx & $\mathrm{L}$ & T2N1M0 & Radical ND & $\mathrm{L}$ & $\mathrm{I}-\mathrm{V}$ \\
\hline 3 & 53 & M & Tongue & $\mathrm{R}$ & T3N1M0 & Radical ND & $\mathrm{R}$ & $\mathrm{I}-\mathrm{V}$ \\
\hline 4 & 62 & $\mathrm{~F}$ & Tongue & $\mathrm{L}$ & T3N0M0 & Selective ND & $\mathrm{L}$ & I, II \\
\hline 5 & 58 & M & Vallecula & $\mathrm{R}$ & T1N0M0 & Selective ND & $\mathrm{RR}$ & I, II, III, V \\
\hline 6 & 40 & M & Tongue & $\mathrm{L}$ & T2N0M0 & Selective ND & $\mathrm{L}$ & I-III \\
\hline 7 & 54 & M & Nasopharynx & $\mathrm{L}$ & T1N1M0 & Selective ND & $\mathrm{L}$ & I-III \\
\hline 8 & 63 & M & Retro molar trigonum & $\mathrm{L}$ & T2N0M0 & Selective ND & $\mathrm{L}$ & I-IV \\
\hline 9 & 55 & $\mathrm{~F}$ & Submandibular gland & $\mathrm{L}$ & T2N0M0 & Selective ND & $\mathrm{L}$ & I, II \\
\hline 10 & 72 & M & Tongue & $\mathrm{L}$ & T2N0M0 & Supraomohyoid ND & $\mathrm{L}$ & I-III \\
\hline 11 & 74 & $\mathrm{~F}$ & Cheek & $\mathrm{L}$ & T2N0M0 & Selective ND & $\mathrm{L}$ & I, II \\
\hline 12 & 51 & $\mathrm{~F}$ & Floor of mouth & $\mathrm{R}$ & T2N0M0 & Radical ND & $\mathrm{R}$ & $\mathrm{I}-\mathrm{V}$ \\
\hline 13 & 52 & $\mathrm{~F}$ & Soft palate & $\mathrm{L}$ & T2N0M0 & Radical ND & $\mathrm{L}$ & $\mathrm{I}-\mathrm{V}$ \\
\hline 14 & 47 & $\mathrm{~F}$ & Tongue & $\mathrm{R}$ & T3N0M0 & Supraomohyoid ND & $\mathrm{R}$ & I-III \\
\hline 15 & 50 & $\mathrm{~F}$ & Tongue & $\mathrm{R}$ & T2N0M0 & Selective ND & $\mathrm{R}$ & I, II \\
\hline \multirow[t]{2}{*}{16} & 77 & M & Nose & - & T2-3N0M0 & Supraomhyoid ND & $\mathrm{R}$ & I-III \\
\hline & & & & & & Supraomohyoid ND & $\mathrm{L}$ & I-III \\
\hline
\end{tabular}

Table 2 Sensitivity, specificity, and diagnostic odds ratio (DOR) with 95\% confidence interval (95\% CI) for the diagnosis of malignant cervical lymph nodes for different threshold values of the apparent diffusion coefficient (ADC)

\begin{tabular}{lccc}
\hline Threshold & Sensitivity $(\%)$ & Specificity (\%) & DOR (95\% CI) \\
\hline $\begin{array}{l}<0.9 \times 10^{-3} \mathrm{~mm}^{2} / \mathrm{sec} \\
\text { versus }>0.9 \times 10^{-3} \mathrm{~mm}^{2} / \mathrm{sec}\end{array}$ & $69.2 \%$ & $92.2 \%$ & $26.7(10.0-71.5)$ \\
$<0.94 \times 10^{-3} \mathrm{~mm}^{2} / \mathrm{sec}$ & $84.6 \%$ & $90.2 \%$ & $50.4(15.7-161.6)$ \\
versus $>0.94 \times 10^{-3} \mathrm{~mm}^{2} / \mathrm{sec}$ & & & \\
$<1.0 \times 10^{-3} \mathrm{~mm}^{2} / \mathrm{sec}$ & $92.3 \%$ & $83.9 \%$ & $62.7(14.1-279)$ \\
versus $>1.0 \times 10^{-3} \mathrm{~mm}^{2} / \mathrm{sec}$ & & & \\
\hline
\end{tabular}


Table 3 Sensitivity, specificity, positive predictive value (PPV) and negative predictive value (NPV) for the diagnosis of malignant lymph nodes for the MR criteria: apparent diffusion coefficient (ADC) $(\leq 1.0 \times 10-3 \mathrm{~mm} 2 / \mathrm{sec}$ versus $>1.0 \times 10-3 \mathrm{~mm} 2 / \mathrm{sec}$ ), size (maximum short axial diameter $\leq 10 \mathrm{~mm}$ versus $>10 \mathrm{~mm}$ ), border irregularity and signal intensity (SI) on T2-weighted images

\begin{tabular}{lcccc}
\hline Criterion & Sensitivity (\%) & Specificity (\%) & PPV (\%) & NPV (\%) \\
\hline $\begin{array}{l}\text { ADC } \\
<1.0 \times 10^{-3} \mathrm{~mm}^{2} / \mathrm{sec}\end{array}$ & $92.3 \%$ & $83.9 \%$ & $43.6 \%$ & $98.8 \%$ \\
$\begin{array}{l}\text { versus }>1.0 \times 10^{-3} \mathrm{~mm}^{2} / \mathrm{sec} \\
\text { Size } \leq 10 \mathrm{~mm} \text { versus }>10 \mathrm{~mm}\end{array}$ & $92.3 \%$ & $60.6 \%$ & $24.0 \%$ & $98.3 \%$ \\
$\begin{array}{l}\text { Border irregularity on T2- } \\
\text { weighted images } \\
\text { spiculated/indistinct } \\
\text { versus smooth/lobulated }\end{array}$ & $61.5 \%$ & $98.3 \%$ & $84.2 \%$ & $94.4 \%$ \\
$\begin{array}{l}\text { SI on T2-weighted images } \\
\text { heterogeneous versus } \\
\text { homogeneous }\end{array}$ & $50.0 \%$ & $94.8 \%$ & & \\
\end{tabular}

Table 4 Diagnostic odds ratios (DORs) with 95\% confidence intervals (95\% CI) for apparent diffusion coefficient (ADC) levels and three assessed morphological criteria (size, border and signal intensity). Results are derived from univariate and multivariate logistic regression analyses

\begin{tabular}{lcc}
\hline MRI criteria & $\begin{array}{c}\text { Univariate analysis } \\
\text { DOR }(95 \% \mathrm{CI})\end{array}$ & $\begin{array}{c}\text { Multivariate analysis } \\
\text { DOR }(95 \% \mathrm{CI})\end{array}$ \\
\hline $\begin{array}{l}\text { ADC threshold } \\
<1.0 \times 10^{-3} \mathrm{~mm}^{2} / \mathrm{sec}\end{array}$ & $62.7(10.0-71.5)$ & $97.6(9.7-982)$ \\
versus $>1.0 \times 10^{-3} \mathrm{~mm}^{2} / \mathrm{sec}$ & & $4.4(0.7-28.0)$ \\
$\begin{array}{l}\text { Size } \leq 10 \mathrm{~mm} \text { versus }>10 \mathrm{~mm} \\
\begin{array}{l}\text { Border irregularity spiculated/indistinct } \\
\text { versus smooth/lobulated }\end{array}\end{array}$ & $18.5(4.2-80.4)$ & $56.0(4.4-719)$ \\
$\begin{array}{l}\text { Signal intensity on T2-weighted image } \\
\text { heterogeneous versus homogeneous }\end{array}$ & $18.2(6.7-49.4)$ & $12.8(1.8-91.6)$ \\
\hline
\end{tabular}

Table 5 Area under the curve (AUC) and 95\% confidence intervals (95\% CI) for predicted probabilities of malignant lymph nodes derived from four models which included different combinations of MRI criteria

\begin{tabular}{llcc}
\hline Model & Criteria & AUC & $95 \%$ CI \\
\hline 1 & ADC (alone) & 0.89 & $0.86-0.92$ \\
2 & size, border and signal intensity on T2-weighted images & 0.91 & $0.84-0.99$ \\
3 & ADC, size, border and signal intensity on T2-weighted images & 0.98 & $0.97-1.00$ \\
4 & size and ADC & 0.94 & $0.91-0.96$ \\
\hline
\end{tabular}



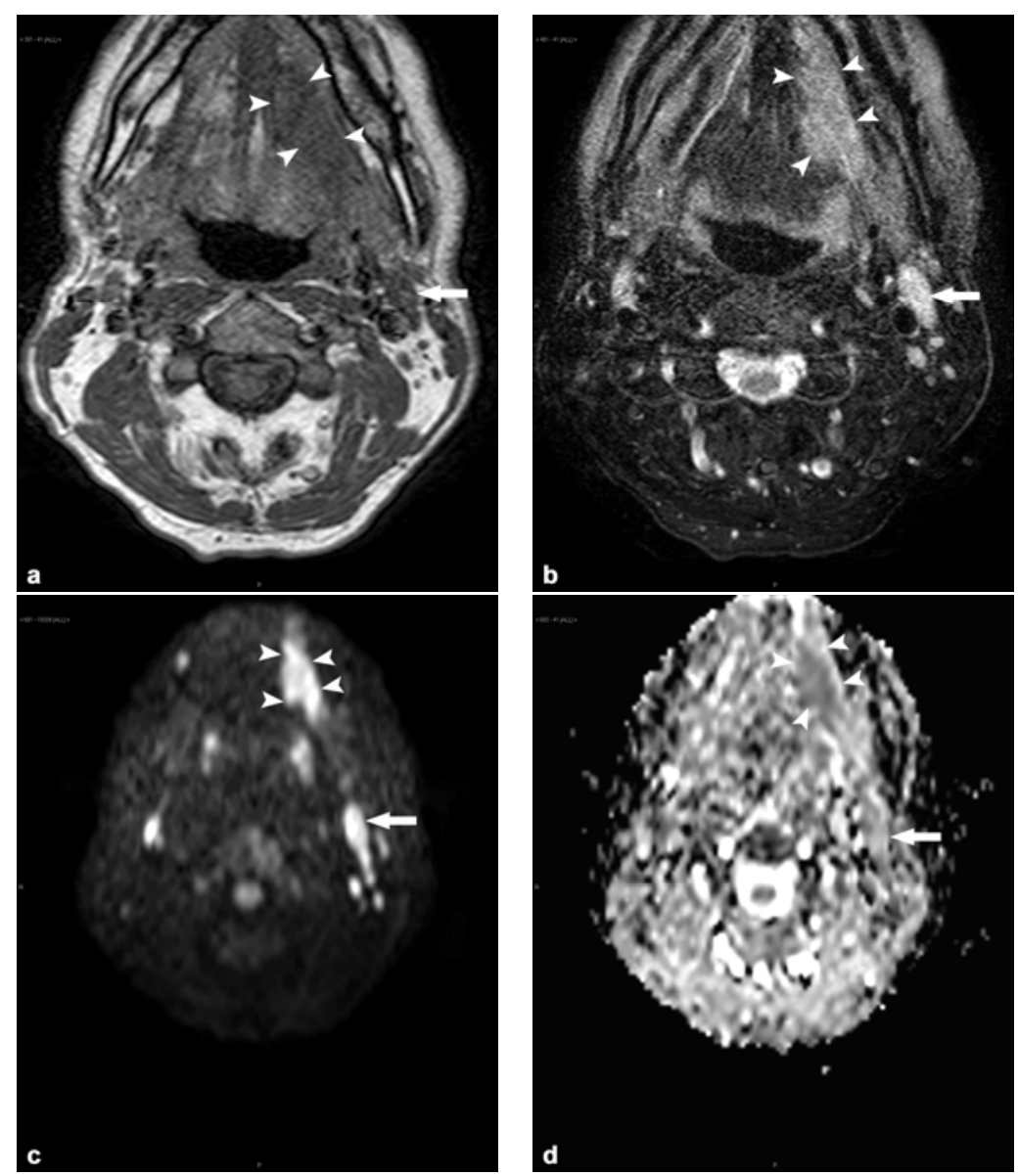

Figure 3 51-year old patient with a squamous cell carcinoma in the floor of the mouth on the left side (arrowheads). The axial T1-weighted (a) and fat suppressed T2-weighted (b) images show in level II on the left side an enlarged lymph node (arrow) with a maximum short axial diameter of $12 \mathrm{~mm}$. This lymph nodes is easily recognized on the diffusion weighted image (DWI) at b1000 (c). The corresponding area on the apparent diffusion coefficient (ADC) map (d) shows a calculated ADC of $1.3 \times 10-3 \mathrm{~mm} 2 / \mathrm{s}$. Pathological examination revealed no metastasis. 


\section{Discussion}

The results show that apparent diffusion coefficient (ADC) values calculated on the diffusion weighted images (DWI), alone or in combination with other morphological criteria, are useful in discriminating malignant from benign lymph nodes in head and neck squamous cell carcinoma (HNSCC). ADC values of malignant lymph nodes were significantly lower compared to benign lymph nodes.

At present there are only few reports that demonstrate the usefulness of DWI-MRI in characterizing lymph nodes in the neck. Some studies reported the ability of DWI to discriminate between different histological lymph node cancers in the neck [53-55]. Others showed that ADC determination on DWI-MRI may have added value in discriminating malignant from benign lymph nodes in the neck [44, 45, 52]. All studies were performed in patients with palpable cervical lymphadenopathy (clinical positive necks), and further evaluation by MRI was limited to selected enlarged lymph nodes.

In contrast to previous reports, this study is unique in that it mainly dealt with patients with clinically negative necks. In only 3 out of 16 patients lymph nodes were palpable at clinical examination, however, in only two patients an enlarged lymph node with maximum short axial diameter ranging from 18 to $30 \mathrm{~mm}$ could also be demonstrated on MRI and at pathological examination. The large majority of lymph nodes (95.4\%) on MRI were $\leq 10 \mathrm{~mm}$, within this group the pathologically confirmed malignant lymph nodes ranged a maximum short axial diameter of $5-10 \mathrm{~mm}$. Those small lymph nodes would all be assigned as benign based on the size criterion. Especially the multivariate regression analyses demonstrate that the size criterion no longer differs significantly malignant from benign lymph nodes and thus has no independent predictive value. When used separately, the border criterion is associated with the highest ability to discriminate between lymph nodes with and without metastasis, however, when used in combination, the ADC criterion is the strongest independent predictor of presence of metastasis followed by the border criterion (Table 4).

These results in the clinically negative necks are of great clinical relevance as DWI shows to be an accurate tool to differentiate metastases in small lymph nodes. In a radiologically negative neck the planned neck dissection could be converted to a wait-and-scan policy, whereas the radiologically determined positive neck would support the decision to perform a neck dissection. The relative high sensitivity and NPV for the size criterion in this study only accounts for lymph nodes exceeding $10 \mathrm{~mm}$ in maximum short axial diameter (Table 3). However, as in this study the 
large majority of lymph nodes were $\leq 10 \mathrm{~mm}$, the size criterion does no longer differ in these lymph nodes between malignant and benign lymph nodes. This is expressed by the low DOR for the size criterion in the univariate and multivariate logistic regression analyses (Table 4).

The optimal ADC threshold for this study population appeared to be $1.0 \times 10^{-3}$ $\mathrm{mm}^{2} / \mathrm{sec}$ (Figure 4); when the criteria size and morphology on the conventional MR protocol were combined with this ADC threshold value, the area under the curve (AUC) significantly increased. Figure 5 illustrates the receiver operating characteristics (ROC) curves derived from predicted probabilities from models including different criteria sets. A model which added ADC values to the other MRI criteria performed significantly better than a model without ADC values whereas the model which includes only ADC performs nearly as well as the model which includes the other three MRI criteria. In a previous study the same assessed morphological criteria showed - in addition to the size criterion - an increased AUC from 0.91 to 0.96 . However, this accounted solely for the experienced head and neck radiologist in a series with higher prevalence of metastases $(27.2 \%)$ and larger assessed lymph nodes $(18 \%>10 \mathrm{~mm})$ [58].

The chosen ADC threshold of $1.0 \times 10^{-3} \mathrm{~mm}^{2} / \mathrm{sec}$ in this study might be difficult to reproduce in other centers. This is probably based on the differences in study design and techniques that will be used and differences in selection of patient series, i.e. the sizes of assessed lymph nodes might influence the choice of the ADC threshold as larger lymph nodes tend to show necrosis and this will affect the ADC values. To use DWI for characterizing lymph nodes in general practice, there should be a well-established threshold for the ADC values. It is advisable that thresholds are reproducible in different centers and on different MR machines, to set the ADC threshold to the proper value depends on the differential diagnosis of expected lymph node pathology.

Some limitations of this study have to be addressed. Firstly, the low prevalence of malignant lymph nodes, with as a result that the negative predictive values were high for all MRI criteria. It must be kept in mind that predictive values are not independent of the setting in which the test is used and are strongly influenced by the prevalence; metastases were present in only 26 out of 219 lymph nodes and negative predictive values of tests tend to be high when applied in a population with a low likelihood of the disease. It also has to be mentioned that the primary tumor sites in this study, which are relatively more likely to show small metastases, have been of influence especially on the size criterion showing low diagnostic accuracy and additional value. Secondly, in 9 out of 45 lymph nodes $(20 \%)$ with a 
size of 3-4 $\mathrm{mm}$ in maximum short axial diameter, ADC values were observed below the threshold of $1.0 \times 10^{-3} \mathrm{~mm}^{2} / \mathrm{sec}$. None of these small lymph nodes showed histopathological evidence of metastasis; partial volume effect when drawing ROIs on the b1000 images around these small lymph nodes might be responsible for the false positive results. Thirdly, although the scan-time of DWI will only take less than 5 minutes, it must be said that evaluating all lymph nodes at the MR workstation is time consuming.

\section{Conclusions}

We demonstrated in a series with predominantly small lymph nodes that ADC values calculated on the diffusion weighted MRI is a strong predictor of the presence of metastasis, independently from other predictors such as size and morphology, in patients with head and neck squamous cell carcinoma (HNSCC). The use of ADC values in combination with the other MRI criteria significantly improves the discrimination between malignant and benign lymph nodes.

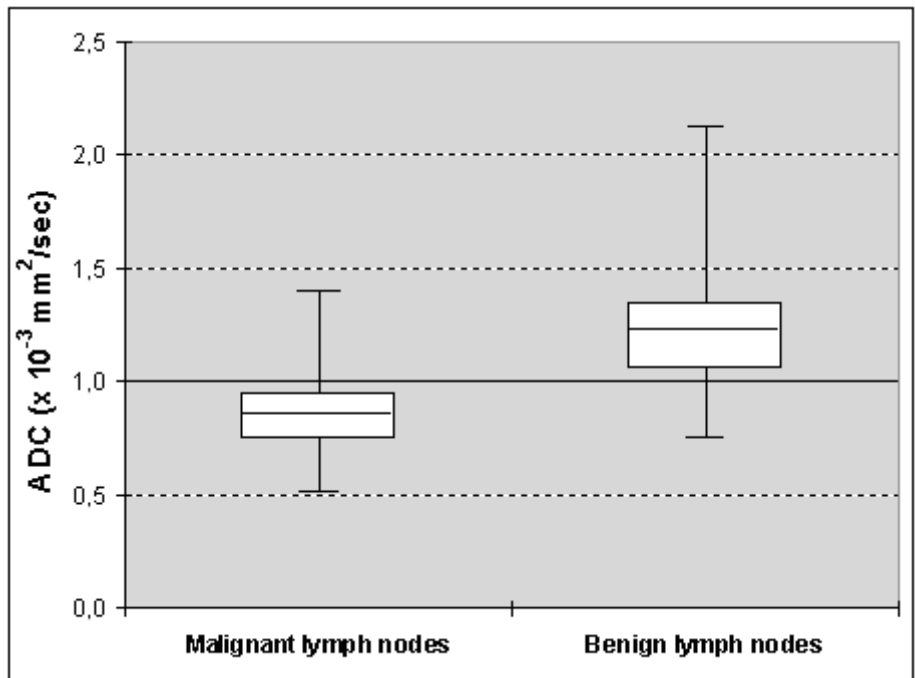

Figure 4 Graph of box plots shows the apparent diffusion coefficient (ADC) values of malignant and benign lymph nodes. The horizontal line in the box represents the median (50th percentile), whereas the top and the bottom represent the 25 th and 75 th percentile, respectively. The whiskers represent the range from the largest and the smallest measured ADC data. The ADC values of benign lymph nodes are higher than compared than those of malignant lymph nodes. 


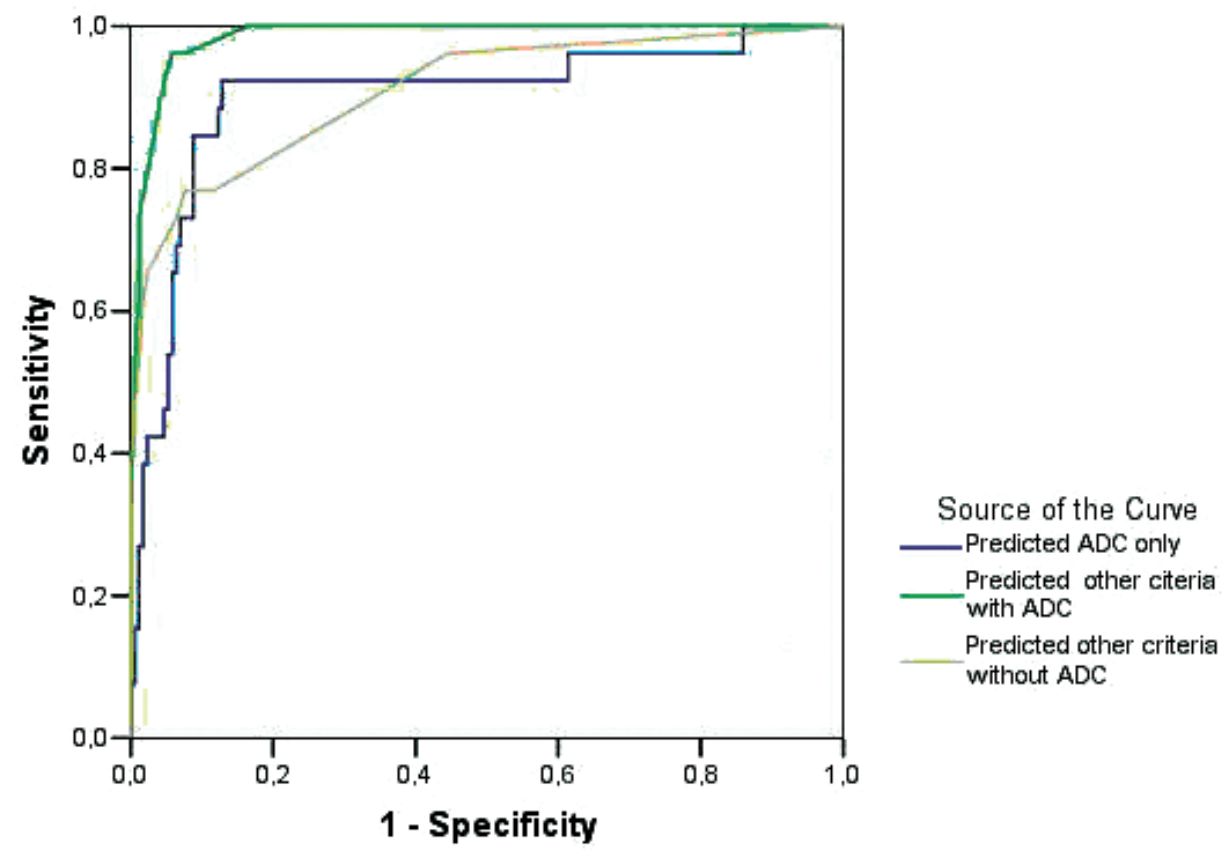

Figure 5 Receiver operating characteristics (ROC) curves derived from predicted probabilities from models including different sets of MRI. A model which added ADC values to the other MRI criteria (green line) performed significantly better than a model without ADC values (gray line): AUC=0.98 versus $\mathrm{AUC}=0.91(\mathrm{p}=0.036)$. Moreover, the model which includes only ADC (blue line) as predictor of presence of metastasis performs nearly as well as the model, which includes the other three MRI criteria as predictors: $\mathrm{AUC}=0.89$ versus $\mathrm{AUC}=0.91(\mathrm{p}=0.52)$. 


\section{References}

1. Straetmans, J.M., et al., Human papillomavirus reduces the prognostic value of nodal involvement in tonsillar squamous cell carcinomas. Laryngoscope, 2009. 119(10): p. 1951-7.

2. Weiss, M.H., L.B. Harrison, and R.S. Isaacs, Use of decision analysis in planning a management strategy for the stage N0 neck. Arch Otolaryngol Head Neck Surg, 1994. 120(7): p. 699-702.

3. de Bondt, R.B., et al., Detection of lymph node metastases in head and neck cancer: A metaanalysis comparing US, USgFNAC, CT and MR imaging. Eur J Radiol, 2007.

4. van den Brekel, M.W., et al., Cervical lymph node metastasis: assessment of radiologic criteria. Radiology, 1990. 177(2): p. 379-84.

5. Cabanas, R.M., An approach for the treatment of penile carcinoma. Cancer, 1977. 39(2): p. 456-66.

6. Tregnaghi, A., et al., Ultrasonographic evaluation of superficial lymph node metastases in melanoma. Eur J Radiol, 1997. 24(3): p. 216-21.

7. Knappe, M., M. Louw, and R.T. Gregor, Ultrasonography-guided fine-needle aspiration for the assessment of cervical metastases. Arch Otolaryngol Head Neck Surg, 2000. 126(9): p. 1091-6.

8. Takes, R.P., et al., The value of ultrasound with ultrasound-guided fine-needle aspiration biopsy compared to computed tomography in the detection of regional metastases in the clinically negative neck. Int J Radiat Oncol Biol Phys, 1998. 40(5): p. 1027-32.

9. van den Brekel, M.W., et al., Modern imaging techniques and ultrasound-guided aspiration cytology for the assessment of neck node metastases: a prospective comparative study. Eur Arch Otorhinolaryngol, 1993. 250(1): p. 11-7.

10. Zenk, J., et al., Neck lymph nodes: prediction by computer-assisted contrast medium analysis? Ultrasound Med Biol, 2007. 33(2): p. 246-53.

11. Curtin, H.D., et al., Comparison of CT and MR imaging in staging of neck metastases. Radiology, 1998. 207(1): p. 123-30.

12. McGuirt, W.F., et al., A comparative diagnostic study of head and neck nodal metastases using positron emission tomography. Laryngoscope, 1995. 105(4 Pt 1): p. 373-5.

13. Steinkamp, H.J., et al., [Reactive enlargement of cervical lymph nodes and cervical lymph node metastases: sonography (M/Q quotient) and computed tomography]. Aktuelle Radiol, 1992. 2(4): p. 188-95.

14. Plaat, R.E., et al., Prognostic importance of paratracheal lymph node metastases. Laryngoscope, 2005. 115(5): p. 894-8.

15. Regelink, G., et al., Detection of unknown primary tumours and distant metastases in patients with cervical metastases: value of FDG-PET versus conventional modalities. Eur J Nucl Med Mol Imaging, 2002. 29(8): p. 1024-30.

16. Myers, L.L., et al., Positron emission tomography in the evaluation of the N0 neck. Laryngoscope, 1998. 108(2): p. 232-6.

17. Schwartz, D.L., et al., FDG-PET/CT imaging for preradiotherapy staging of head-and-neck squamous cell carcinoma. Int J Radiat Oncol Biol Phys, 2005. 61(1): p. 129-36.

18. Herborn, C.U., et al., Interstitial MR lymphography with MS-325: characterization of normal and tumor-invaded lymph nodes in a rabbit model. AJR Am J Roentgenol, 2002. 179(6): p. 1567-72.

19. Misselwitz, B., J. Platzek, and H.J. Weinmann, Early MR lymphography with gadofluorine M in rabbits. Radiology, 2004. 231(3): p. 682-8.

20. Weissleder, R., et al., Ultrasmall superparamagnetic iron oxide: an intravenous contrast agent for assessing lymph nodes with MR imaging. Radiology, 1990. 175(2): p. 494-8. 
21. Anzai, Y., et al., Dextran-coated superparamagnetic iron oxide, an MR contrast agent for assessing lymph nodes in the head and neck. AJNR Am J Neuroradiol, 1994. 15(1): p. 87-94.

22. Will, O., et al., Diagnostic precision of nanoparticle-enhanced MRI for lymph-node metastases: a meta-analysis. Lancet Oncol, 2006. 7(1): p. 52-60.

23. Kvistad, K.A., et al., Axillary lymph node metastases in breast cancer: preoperative detection with dynamic contrast-enhanced MRI. Eur Radiol, 2000. 10(9): p. 1464-71.

24. Fischbein, N.J., et al., Assessment of metastatic cervical adenopathy using dynamic contrastenhanced MR imaging. AJNR Am J Neuroradiol, 2003. 24(3): p. 301-11.

25. de Lussanet, Q.G., et al., Dynamic contrast-enhanced magnetic resonance imaging of radiation therapy-induced microcirculation changes in rectal cancer. Int J Radiat Oncol Biol Phys, 2005. 63(5): p. 1309-15.

26. Liney, G.P., et al., Dynamic contrast-enhanced MRI in the differentiation of breast tumors: user-defined versus semi-automated region-of-interest analysis. J Magn Reson Imaging, 1999. 10(6): p. 945-9.

27. Kalnins, I.K., et al., Correlation between prognosis and degree of lymph node involvement in carcinoma of the oral cavity. Am J Surg, 1977. 134(4): p. 450-4.

28. Jakobsen, J., et al., Lymph node metastases from laryngeal and pharyngeal carcinomas-calculation of burden of metastasis and its impact on prognosis. Acta Oncol, 1998. 37(5): p. 489-93.

29. Kehrl, W., S. Wenzel, and A. Niendorf, [Effect of various forms of metastatic lymph node involvement on prognosis of squamous epithelial carcinomas of the upper aerodigestive tract]. Laryngorhinootologie, 1998. 77(10): p. 569-75.

30. Ganzer, U., et al., [Effect of tumor size on lymph node metastasis and type of treatment on the prognosis of hypopharyngeal cancer]. Laryngol Rhinol Otol (Stuttg), 1982. 61(11): p. 622-8.

31. Leemans, C.R., et al., Recurrence at the primary site in head and neck cancer and the significance of neck lymph node metastases as a prognostic factor. Cancer, 1994. 73(1): p. 18790.

32. Leemans, C.R., et al., [Neck lymph node dissection in squamous cell carcinoma originating in the head-and-neck area; the significance for the prognosis]. Ned Tijdschr Geneeskd, 1992. 136(5): p. 221-5.

33. Foote, R.L., K.D. Olsen, and D.L. Davis, Base of tongue carcinoma: patterns of failure and predictors of recurrence after surgery alone. Head Neck, 1993. 15(4): p. 300-7.

34. Taylor, J.M., W.M. Mendenhall, and R.S. Lavey, Time-dose factors in positive neck nodes treated with irradiation only. Radiother Oncol, 1991. 22(3): p. 167-73.

35. King, A.D., et al., Necrosis in metastatic neck nodes: diagnostic accuracy of CT, MR imaging, and US. Radiology, 2004. 230(3): p. 720-6.

36. de Bondt, R.B., et al., Detection of lymph node metastases in head and neck cancer: a metaanalysis comparing US, USgFNAC, CT and MR imaging. Eur J Radiol, 2007. 64(2): p. 26672.

37. Anzai, Y., et al., Evaluation of neck and body metastases to nodes with ferumoxtran 10enhanced MR imaging: phase III safety and efficacy study. Radiology, 2003. 228(3): p. 777-88.

38. Sigal, R., et al., Lymph node metastases from head and neck squamous cell carcinoma: MR imaging with ultrasmall superparamagnetic iron oxide particles (Sinerem MR) -- results of a phase-III multicenter clinical trial. Eur Radiol, 2002. 12(5): p. 1104-13.

39. Baghi, M., et al., The efficacy of MRI with ultrasmall superparamagnetic iron oxide particles (USPIO) in head and neck cancers. Anticancer Res, 2005. 25(5): p. 3665-70.

40. Baghi, M., et al., Iron oxide particle-enhanced magnetic resonance imaging for detection of benign lymph nodes in the head and neck: how reliable are the results? Anticancer Res, 2007. 27(5B): p. 3571-5. 
41. Mack, M.G., et al., Superparamagnetic iron oxide-enhanced MR imaging of head and neck lymph nodes. Radiology, 2002. 222(1): p. 239-44.

42. van den Brekel, M.W., J.A. Castelijns, and G.B. Snow, The size of lymph nodes in the neck on sonograms as a radiologic criterion for metastasis: how reliable is it? AJNR Am J Neuroradiol, 1998. 19(4): p. 695-700.

43. van den Brekel, M.W., et al., Occult metastatic neck disease: detection with US and US-guided fine-needle aspiration cytology. Radiology, 1991. 180(2): p. 457-61.

44. Wang, J., et al., Head and neck lesions: characterization with diffusion-weighted echo-planar MR imaging. Radiology, 2001. 220(3): p. 621-30.

45. Koc, O., et al., Role of diffusion weighted MR in the discrimination diagnosis of the cystic and/or necrotic head and neck lesions. Eur J Radiol, 2007. 62(2): p. 205-13.

46. Kato, H., et al., Head and neck squamous cell carcinoma: usefulness of diffusion-weighted MR imaging in the prediction of a neoadjuvant therapeutic effect. Eur Radiol, 2008.

47. Kito, S., et al., Utility of diffusion-weighted images using fast asymmetric spin-echo sequences for detection of abscess formation in the head and neck region. Oral Surg Oral Med Oral Pathol Oral Radiol Endod, 2006. 101(2): p. 231-8.

48. Vandecaveye, V., et al., Detection of head and neck squamous cell carcinoma with diffusion weighted MRI after (chemo)radiotherapy: correlation between radiologic and histopathologic findings. Int J Radiat Oncol Biol Phys, 2007. 67(4): p. 960-71.

49. Vandecaveye, V., et al., Evaluation of the larynx for tumour recurrence by diffusion-weighted MRI after radiotherapy: initial experience in four cases. Br J Radiol, 2006. 79(944): p. 681-7.

50. Abdel Razek, A.A., et al., Role of diffusion-weighted echo-planar MR imaging in differentiation of residual or recurrent head and neck tumors and posttreatment changes. AJNR Am J Neuroradiol, 2007. 28(6): p. 1146-52.

51. Takahara, T., et al., Diffusion weighted whole body imaging with background body signal suppression (DWIBS): technical improvement using free breathing, STIR and high resolution 3D display. Radiat Med, 2004. 22(4): p. 275-82.

52. Sumi, M., et al., Discrimination of metastatic cervical lymph nodes with diffusion-weighted MR imaging in patients with head and neck cancer. AJNR Am J Neuroradiol, 2003. 24(8): p. 1627-34.

53. Sumi, M., M. Van Cauteren, and T. Nakamura, MR microimaging of benign and malignant nodes in the neck. AJR Am J Roentgenol, 2006. 186(3): p. 749-57.

54. Abdel Razek, A.A., et al., Role of diffusion-weighted $M R$ imaging in cervical lymphadenopathy. Eur Radiol, 2006. 16(7): p. 1468-77.

55. King, A.D., et al., Malignant cervical lymphadenopathy: diagnostic accuracy of diffusionweighted MR imaging. Radiology, 2007. 245(3): p. 806-13.

56. Fleming, I., J. Cooper, and D. Henson, AJCC Staging manual. Lippenscott-Raven, 1997. 5th ed. Philadelphia.

57. Hanley, J.A. and B.J. McNeil, A method of comparing the areas under receiver operating characteristic curves derived from the same cases. Radiology, 1983. 148(3): p. 839-43.

58. de Bondt RBJ, H.M., Nelemans PJ, Deserno WMLLG, Peutz-Kootstra C, Kremer B, BeetsTan RGH, Morphologic MRI criteria improve the detection of lymph node metastases in head and neck squamous cell carcinoma: multivariate logistic regression analysis of MRI features of cervical lymph nodes. European Radiology 2007;64:266-272. 


\section{CHAPTER 7}

General discussion and future perspectives 


\section{General discussion}

The objectives of this thesis were to evaluate the diagnostic performance of MR imaging for the detection of metastatic lymph nodes in the neck in patients presenting with HNSCC and to explore how accuracy of diagnosis by MRI can be improved. The prognosis of patients with HNSCC is mainly determined by the presence or absence of lymph node metastases. Therefore, the identification of lymph node disease by radiological imaging methods remains a challenge for each radiologist working in the diagnostic oncology field. Despite several attempts regarding imaging and therapy that have been made in the last decades, the prognosis of head and neck cancers has not really been improved. Part of the lack of improvement can be contributed to the fact that precise stratification of patients to more or less aggressive treatments is not possible, as staging by imaging lacks sufficient accuracy.

\section{Evaluation of diagnostic accuracy of $M R I$}

Prediction of lymph node involvement with the currently available radiological imaging modalities cannot be reliably used for clinical decision making, unless combined with invasive and demanding procedures such as ultrasound guided fine needle aspiration cytology (USgFNAC).

However, there are some drawbacks regarding the use of USgFNAC. This technique is not only time consuming, but also operator dependent and subject to false negative results due to sampling errors. The reported variation in sensitivity of $48-97 \%$ might indicate that USgFNAC is less reproducible in general centers. Another limitation is that lymph nodes located in the retropharyngeal space are beyond the scope of the US probe. Unlike USgFNAC, MRI is non-invasive and less dependent on the operator experience. MRI provides a roadmap before treatment and can be helpful for radiotherapists and surgeons to plan their treatment approach. A further advantage, as compared with CT and PET-CT, is that the patient is not exposed to radiation. Therefore, MRI could become an excellent imaging tool for detection of metastases in cervical lymph nodes if sensitivity and specificity could reach levels acceptable for clinical decision making.

\section{Additional value of morphological criteria}

The finding that USgFNAC is superior to US, CT and MRI was confirmed in a meta-analysis of 17 published studies (Chapter 3). US performed better than CT and MRI. MRI without the use of the contrast agent USPIO was associated with poor performance. According to the published literature, MRI is not sufficiently 
accurate to exclude the presence of occult metastases. Sensitivity and specificity of MRI definitely need to be improved. The poor performance of MRI may partly be explained by the consideration that the mere use of size as a criterion to classify lymph nodes as benign or malignant is inadequate. Sensitivity is poor when using the commonly used cut-off value of $10 \mathrm{~mm}$, and although use of a lower cut-off value results in higher sensitivity, lower cut-off values are associated with low specificity. Also the use of other criteria, such as measurements of ratio in dimensions and morphological aspects of lymph nodes, was expected to decrease the probability that small metastatic lymph nodes will be missed. The results presented in Chapter 5 indicate that morphological criteria, like border irregularity and heterogeneity of signal intensity on T2-weighted images, in addition to the size criterion significantly improved the prediction of the presence of cervical lymph node metastases in patients with HNSCC. All criteria evaluated and used as a stand-alone, helped to discriminate between nodes with and without metastasis. However, the study showed that diagnostic odds ratios (DORs) associated with the combined use of all morphological criteria were the best, and in particular for the specialized radiologist. This finding indicates that experience in head and neck imaging is required for adequate use of morphological criteria.

The results in Chapter 5 also show that after restriction of the analysis to lymph nodes smaller than $10 \mathrm{~mm}$, the morphological criteria were still useful, but discriminative ability was poorer. Reductions in sensitivity were only minor for the experienced MRI reader, but for the less experienced reader sensitivity reductions were more substantial: from $63 \%$ to $40 \%$ for border irregularity and $67 \%$ to $53 \%$ for signal heterogeneity on T2-weighted images, respectively. An explanation for these findings may be that an experienced observer is less focused on size and better aware of the diagnostic value of other criteria. As a result, border irregularity and signal heterogeneity on T2-weighted images are more decisive in the diagnosis; this was also reflected by the results from univariate analyses with higher DORs for these criteria $(\mathrm{DOR}=112$ and $\mathrm{DOR}=28.6$, respectively) when compared with the low DOR associated with size $(\mathrm{DOR}=8)$.

\section{Additional value of other MRI sequences: STIR and DWI}

This thesis also explored the additional value of the use of other MRI sequences and newer functional MR techniques, such as the STIR and DWI.

The results presented in chapter 4 indicate that the use of the STIR sequence compared to the size criterion on conventional MRI improved the detection of cervical metastatic lymph nodes in HNSCC. It has to be emphasized that the sole finding of pathological hypo-intense signal on the reversed STIR images will not be sufficient to determine lymph nodes as malignant: STIR is highly sensitive for the 
detection of pathologic lesions, but it is not specific for malignancy alone as a hypo-intense signal can also be observed in inflammatory lymph nodes. However, this finding on STIR is an important indicator of pathological lymph nodes, and the STIR sequence has added value as it could be used as a pathfinder for visualization and detection of existing lymph nodes. Subsequently, other available MRI sequences and morphological criteria should be reviewed for the decision to determine detected lymph nodes on STIR to be malignant.

Use of the diffusion weighted imaging (DWI) sequence provides quantitative functional information on nodes by measuring the apparent diffusion coefficient (ADC). The use of ADC values in combination with other MRI criteria significantly improved the discrimination between malignant and benign lymph nodes. A model which added ADC values to the other predictors performed significantly better than a model without $\mathrm{ADC}$ values (AUC $=0.98$ versus AUC $=0.91, p=0.036$ ). Other studies have shown that determination of ADC values may have added value to conventional MRI in discriminating malignant form benign nodes in the neck. However, these studies were all performed in patients with palpable nodes at clinical examination (clinically positive necks). The study described in Chapter 6 is unique in that it mainly dealt with patients with clinically negative necks. In this clinically relevant population, use of the ADC value resulted in more accurate diagnosis than use of the size criterion (sensitivity/specificity for ADC was $92 \% / 84 \%$ compared with $92 \% / 61 \%$ for size). Based on these results, the use of DWI-MRI seems a promising technique for detection of lymph node involvement in patients with HNSCC. If future studies can confirm these results, non-invasive DWI-MRI might potentially be able to replace the more invasive USgFNAC modality. At present, the measurements of $\mathrm{ADC}$ values are time consuming and not suitable for implementation in general practices, unless studies will focus on automatically acquisition of the ADC data. Future studies should also address the question whether the ADC threshold value of $1.0 \times 10^{-3} \mathrm{~mm}^{2} / \mathrm{sec}$, which was used in this study to differentiate between benign and malignant lymph nodes, is optimal for use in other study populations which may differ in distribution with respect to clinically positive and negative necks.

\section{Diagnosis and management of patients with clinically negative necks}

Diagnosis of cervical lymph node metastases in patients with clinically N0 necks constitutes the main challenge, because for these patients there is much at stake. On the one hand, inability to completely rule out the presence of lymph node metastases by radiological imaging implies that there is a risk that occult metastases remain undetected and that the patient is undertreated, which has negative repercussion for the prognosis of these patients. On the other hand, a 
"wait-and-see policy", where it is decided to refrain from elective dissection of lymph nodes, has the advantage that unnecessary morbidity due to surgery can be avoided in patients who are free from cervical lymph node metastases. Especially in this group, evaluation of the accuracy of radiological imaging modalities is clinically relevant and therefore, it is surprising that studies in patients with N0 necks are almost absent from the literature. The literature search that was required for the meta-analysis in Chapter 3 revealed that studies, which explicitly address the evaluation of the clinically N0 necks, are scarce. In the literature, only three such studies could be found and two of these studies were included in the review. Only one study evaluated diagnostic performance of MRI in patients with N0 necks [1]. This study used size and central necrosis as criteria for the differentiation between malignant and benign nodes and quite surprisingly found acceptable estimates of sensitivity and specificity ( $82 \%$ and $81 \%$, respectively). The diagnostic odds ratio, which combines sensitivity and specificity into one measure, was 19 and corresponds well with the results in Chapter 5, where size was associated with a sensitivity of $92 \%$, a specificity of $61 \%$ and a DOR $=18$. These DORs compare favorably with the mean DOR for MRI that was estimated at DOR=7 in the metaanalysis. This finding however raises the question whether the size criterion performs better in patients with $\mathrm{N} 0$ necks than in patients with $\mathrm{N}+$ necks or whether these results occurred by chance. Furthermore, it is important to evaluate whether the diagnostic gain, which can be achieved by using other criteria than size in patients with N0 necks, is comparable to that in patients with palpable cervical lymph nodes. Future studies should focus more on diagnostic performance of the combined use of MRI features in patients with N0 necks to provide the answers. The consideration that we need answers to clinically relevant questions should outweigh objections that, due to lower prevalence of lymph node metastases, smaller sample sizes will be available for estimation of sensitivity than in series with $\mathrm{N}+$ necks.

\section{Future perspectives}

The role in future of the blood pool contrast agent Ultra small Super Paramagnetic Iron Oxide (USPIO) is questionable as USPIO is not FDA approved in Europe nor at the other side of the Atlantic. Another MR blood pool contrast agent, Gadofosveset, (Vasovist Bayer Schering Pharma) has shown potential in preliminary studies in rectal cancer patients; however at the moment of writing, this contrast is not marketed in Europe but only available on the market in the USA. [2] 
There is early experience indicating that DWI may not only be helpful for detection and staging of the primary tumor and regional lymph node metastases, but also for detection of distant metastasis. Especially Whole Body DWI (DWIBS) seems to be particularly promising and even shows the potential to compete with the results of PET-CT. DWIBS is a method without the use of intravenous contrast agent and without the use of irradiation[3]

Developments in the field of combined imaging, such as the integrated PET-CT and PET-MRI machines, in which functional and morphological information are combined, will probably further refine the assessment of the neck [4-13].

It is very likely to assume that the MRI sequences STIR and DWI, combined with the morphological criteria border irregularity and heterogeneous signal will provide the highest diagnostic performance. A prospective study is needed to prove this assumption. 


\section{References}

1 van den Brekel, M. W., Castelijns, J. A., Stel, H. V., et al., "Modern imaging techniques and ultrasound-guided aspiration cytology for the assessment of neck node metastases: a prospective comparative study." Eur Arch Otorhinolaryngol. 1993. 250(1): 11-7.

2 Herborn, C. U., Lauenstein, T. C., Vogt, F. M., et al., "Interstitial MR lymphography with MS-325: characterization of normal and tumor-invaded lymph nodes in a rabbit model." AJR Am J Roentgenol. 2002. 179(6): 1567-72.

3 Kwee, T. C., Takahara, T., Ochiai, R., et al., "Diffusion-weighted whole-body imaging with background body signal suppression (DWIBS): features and potential applications in oncology." Eur Radiol. 2008. 18(9): 1937-52.

4 Connell, C. A., Corry, J., Milner, A. D., et al., "Clinical impact of, and prognostic stratification by, F-18 FDG PET/CT in head and neck mucosal squamous cell carcinoma." Head Neck. 2007. 29(11): 986-95.

5 Escott, E. J., "Positron emission tomography-computed tomography protocol considerations for head and neck cancer imaging." Semin Ultrasound CT MR. 2008. 29(4): 263-70.

6 Fleming, A. J., Jr. and Johansen, M. E., "The clinician's expectations from the use of positron emission tomography/computed tomography scanning in untreated and treated head and neck cancer patients." Curr Opin Otolaryngol Head Neck Surg. 2008. 16(2): 127-34.

7 Kyzas, P. A., Evangelou, E., Denaxa-Kyza, D., et al., "18F-fluorodeoxyglucose positron emission tomography to evaluate cervical node metastases in patients with head and neck squamous cell carcinoma: a meta-analysis." J Natl Cancer Inst. 2008. 100(10): 712-20.

8 Nahmias, C., Carlson, E. R., Duncan, L. D., et al., "Positron emission tomography/computerized tomography (PET/CT) scanning for preoperative staging of patients with oral/head and neck cancer." J Oral Maxillofac Surg. 2007. 65(12): 2524-35.

$9 \mathrm{Ng}, \mathrm{S}$. H., Yen, T. C., Liao, C. T., et al., "18F-FDG PET and CT/MRI in oral cavity squamous cell carcinoma: a prospective study of 124 patients with histologic correlation." J Nucl Med. 2005. 46(7): 1136-43.

10 Pentenero, M., Cistaro, A., Brusa, M., et al., "Accuracy of 18F-FDG-PET/CT for staging of oral squamous cell carcinoma." Head Neck. 2008. 30(11): 1488-96.

11 Quon, A., Fischbein, N. J., McDougall, I. R., et al., "Clinical role of 18F-FDG PET/CT in the management of squamous cell carcinoma of the head and neck and thyroid carcinoma." J Nucl Med. 2007. 48 Suppl 1: 58S-67S.

12 Roh, J. L., Yeo, N. K., Kim, J. S., et al., "Utility of 2-[18F] fluoro-2-deoxy-D-glucose positron emission tomography and positron emission tomography/computed tomography imaging in the preoperative staging of head and neck squamous cell carcinoma." Oral Oncol. 2007. 43(9): 887-93.

13 Veit-Haibach, P., Luczak, C., Wanke, I., et al., "TNM staging with FDG-PET/CT in patients with primary head and neck cancer." Eur J Nucl Med Mol Imaging. 2007. 34(12): 1953-62. 
$\longrightarrow$ 


\section{CHAPTER 8}

Conclusions and Recommendations 


\section{Conclusions}

According to a meta-analysis comparing USgFNAC, US, CT and MRI with respect to diagnostic accuracy for the detection of cervical lymph node metastases, conventional MRI had the worst diagnostic performance. MRI was not accurate enough to rule out the presence of (occult) metastases.

The use of the STIR sequence improves the diagnostic accuracy of MRI, and the STIR sequence has added value as it could be used as a pathfinder for visualization and detection of existing lymph nodes. A hypo-intense signal intensity of lymph nodes on STIR is an important, but not specific, indicator of pathology. Subsequently, other MRI sequences and morphological criteria should be used for the decision to determine lymph nodes to be malignant.

The use of morphological criteria for assessment of lymph nodes assessed on T2 weighted images in addition to size significantly improved the prediction of the presence of cervical lymph node metastases in patients with HNSCC. However, experience in head and neck imaging is required for adequate use of the assessed morphological criteria.

The use of the diffusion weighted imaging (DWI) sequence provides information on the apparent diffusion coefficient (ADC) of different tissues and lesions. ADC values of malignant lymph nodes were significantly lower compared with benign lymph nodes. The optimal cut-off value for ADC was $1.0 \times 10^{-3} \mathrm{~mm}^{2} / \mathrm{sec}$. The use of ADC values in combination with other MRI criteria significantly improved the discrimination between malignant and benign lymph nodes in patients with predominantly clinically negative necks. ADC measurements are time consuming and at present not yet ready for clinical implementation in a busy radiological practice.

The results on the predictive value of MRI features for the presence of lymph node metastases have to be validated in other studies;other populations and prediction models, which assign weights to the scores on different criteria, have to be developed.

Diagnosis of cervical lymph node metastases in patients with N0 necks constitutes the main challenge, but studies that explicitly address the evaluation of the clinically N0 necks are scarce. Future studies should focus on diagnostic performance of the combined use of MRI features in patients with clinically negative necks. 


\section{Recommendations}

At present, no difference in MRI protocol should be made for the clinically N0 or clinically $\mathrm{N}+$ necks.

The pretherapeutic work up of patients with HNSCC should preferably be performed in expert centers as experience is essential when using the morphological criteria. Calculation of ADC values derived from the DWI sequence is time consuming and not yet suitable in peripheral settings. Furthermore, there is more research and evidence needed to establish the added diagnostic value of DWI.

The MRI protocol - focussed on the assessment of both the primary tumor and the neck nodes - is recommended to include the STIR sequence as a road map of lymph nodes, subsequently morphological criteria should be assessed on fat suppressed T2 weighted images, and the DWI sequence should be performed to calculate the ADC values. A combined assessment of MRI sequences and criteria is recommended to achieve the highest diagnostic performance. 
$\longrightarrow$ 


\section{CHAPTER 9}

Summary 


\section{Summary}

In this thesis the diagnostic value of MRI relative to that of other imaging methods is discussed with regard to the differentiation of malignant from benign cervical lymph nodes in patients with head and neck squamous cell carcinoma (HNSCC). The focus is on the use of new sequences and/or other MRI criteria to improve the diagnostic performance.

In Chapter 2 the anatomy and physiology of lymph nodes are described. Furthermore, this chapter aimed at describing the present status of modern imaging techniques for identifying lymph node disease in patients with head and neck cancer. An overview is given of all applicable radiological imaging modalities in which for every modality the present diagnostic accuracy is discussed.

Chapter 3 presented a meta-analysis in which the diagnostic performance of the radiological imaging modalities US, USgFNAC, CT, and MRI are compared for the detection of cervical lymph node metastases in patients with HNSCC. USgFNAC shows to be the most reliable imaging technique to assess the presence of metastases in cervical lymph nodes. Ultrasound alone is associated with a lower diagnostic performance, this is followed by CT. Conventional MRI shows to be the least performing imaging modality. Furthermore, some advantages and disadvantages of each imaging modality are discussed.

In Chapter 4 an analysis is performed on the role of the STIR sequence over the size criterion on the conventional MRI for the detection of cervical lymph nodes metastases in HNSCC. The use of the STIR sequence improves the diagnostic accuracy of MRI, and the STIR sequence has added value as it could be used as a pathfinder for visualization and detection of existing lymph nodes. STIR is a sensitive and fast MRI sequence and adding STIR to the conventional MRI protocol is recommended.

Chapter 5 presents a study on the use of morphological criteria for the detection of cervical lymph node metastases in patients with HNSCC. The morphological criteria border irregularity and heterogeneity of signal intensity assessed on T2weighted images, in addition to the size criterion on the conventional MRI, 
significantly improve the detection of cervical lymph node metastases. Experience in assessment of cervical lymph nodes is required to adequately use these morphological criteria.

In Chapter 6 is investigated the use of Diffusion Weighted Imaging (DWI) and Apparent Diffusion Coefficient (ADC) in patients with HNSCC. In a series with predominantly small lymph nodes, ADC values calculated based on the diffusionweighted MRI appeared to be a strong predictor of the presence of metastasis, this was independent from other assessed predictors such as size and morphological criteria. The optimal ADC threshold to differentiate between malignant and benign lymph nodes in this evaluated study population appeared to be $1.0 \times 10-3$ $\mathrm{mm} 2 / \mathrm{sec}$. From the results it can be concluded that the use of ADC values, in combination with the other assessed MRI criteria, significantly improves the discrimination between malignant and benign cervical lymph nodes.

Chapter 7 provides a general discussion and future perspectives are discussed.

Chapter 8 focuses on the main conclusions and recommendation for MR imaging are provided. 
$\longrightarrow$ 
CHAPTER 10

Samenvatting 


\section{Samenvatting}

In dit proefschrift wordt de diagnostische waarde beschreven van MRI ten opzichte van andere beeldvormende technieken met de focus op de differentiatie van maligne van benigne lymfeklieren in de hals bij patiënten met een plaveiselcelcarcinoom in het hoofd-hals gebied. De nadruk ligt op het gebruik van nieuwe MRI sequenties en/of het gebruik van morfologische criteria voor de beoordeling van lymfeklieren, met als doel om de diagnostische waarde van MRI te verbeteren.

Hoofdstuk 2 behandelt de anatomie en fysiologie van lymfeklieren. Het doel van dit hoofdstuk is het beschrijven van de actuele status van de beeldvormende technieken voor de detectie van lymfekliermetastasen bij hoofd-hals kanker. Er wordt een overzicht gegeven van alle beschikbare radiologische beeldvormende technieken, waarbij voor elke modaliteit de huidige diagnostische waarde wordt beschreven.

Hoofdstuk 3 bespreekt een meta-analyse, waarbij de radiologische modaliteiten echografie (US), echografie geleide cytologische punctie (USgFNAC), CT en MRI worden vergeleken, en de diagnostische waarden worden bepaald, voor de detectie van lymfekliermetastasen in de hals bij patiënten met een plaveiselcelcarcinoom in het hoofd-hals gebied. USgFNAC blijkt hiervoor de meest betrouwbare beeldvormende techniek te zijn. Echografie alleen, dus zonder cytologische punctie, heeft een lagere diagnostische waarde, gevolgd door CT. Conventioneel MRI onderzoek is de modaliteit met de laagste diagnostische waarde. Enkele voor- en nadelen van elke beeldvormende techniek worden in dit hoofdstuk nader besproken.

Hoofdstuk 4 beschrijft een studie waarbij de rol van de STIR sequentie, ten opzichte van het groottecriterium bij het conventionele MRI onderzoek, voor de detectie van lymfekliermetastasen in de hals bij patiënten met een hoofdhals plaveiselcelcarcinoom is geanalyseerd. Het gebruik van STIR verbetert de diagnostische waarde van MRI. Verder heeft STIR een toegevoegde waarde als signaalfunctie bij de detectie van lymfeklieren in de hals; deze kunnen vervolgens ook worden beoordeeld op basis van andere criteria. STIR is een sensitieve en 
snelle MRI sequentie. De toevoeging van de STIR sequentie aan het conventionele scanprotocol wordt daarom aanbevolen.

Hoofdstuk 5 betreft een studie naar het gebruik van morfologische criteria ten behoeve van de detectie van lymfekliermetastasen in de hals bij patiënten met een plaveiselcelcarcinoom in het hoofd-hals gebied. De morfologische criteria met betrekking tot onregelmatige afgrensbaarheid van de lymfeklier en heterogeniteit van de Signaal Intensiteit van lymfeklieren, die het best op de T2-gewogen MRI beelden kunnen worden beoordeeld, worden vergeleken met het grootte criterium op het conventionele MRI onderzoek. Het gebruik van deze criteria leidt tot een significante verbetering van de detectie van lymfekliermetastasen. Deze beoordeling vereist echter ruime ervaring om deze criteria adequaat te kunnen toepassen.

In hoofdstuk 6 is wordt het gebruik van Diffusion Weighted Imaging (DWI) en de Apparent Diffusion Coefficient (ADC) besproken. Uit de resultaten blijkt dat een groot deel van de patiënten een klinisch negatieve hals hadden en het merendeel van de beoordeelde lymfklieren kleiner dan $1 \mathrm{~cm}$ in doorsnede waren. De ADC waarde - berekend op basis van de DWI gewogen beelden- blijkt een sterke voorspeller te zijn van de aanwezigheid van metastasen in lymfeklieren. Dit resultaat is onafhankelijk van de andere variabelen zoals de criteria grootte en morfologie. De optimale ADC drempelwaarde voor de differentiatie tussen maligne en benigne lymfeklieren in deze studiepopulatie wordt bepaald op $1.0 \times 10^{-}$ ${ }^{3} \mathrm{~mm}^{2} / \mathrm{sec}$. Uit deze resultaten kan worden geconcludeerd, dat het gebruik van de ADC in combinatie met de andere MRI criteria het onderscheidend vermogen aanzienlijk verbetert tussen maligne en benigne lymfklieren.

Hoofdstuk 7 betreft de algemene discussie, waarbij er, naast de gevonden resultaten, ook enkele overwegingen worden gegeven ten aanzien van toekomstig onderzoek.

In Hoofdstuk 8 worden de conclusies van dit proefschrift beschreven en worden er aanbevelingen gedaan ten aanzien van de MR beeldvorming van de hals. 
$\longrightarrow$ 


\section{Dankwoord}

Een promotieonderzoek gaat zeker niet vanzelf, dat geldt ook minstens voor de afronding daarvan. Met veel enthousiasme ben ik in 2006 gestart met het onderwerp MR beeldvorming van halsklieren bij hoofdhals tumoren, resulterend in dit proefschrift. De totstandkoming hiervan heeft afgehangen van vele betrokkenen, in de eerste plaats de medewerking van alle patiënten waarvoor ik hen graag wil bedanken. Voorts zijn er vele betrokkenen in mijn werk en privé omgeving geweest die het mogelijk hebben gemaakt om voldoende tijd te kunnen besteden aan dit onderzoek.

In gedachten ben ik niemand vergeten, hoogstens wordt nu niet iedereen genoemd.

Prof. dr. R.G.H. Beets-Tan, promotor. Beste Regina, tijdens mijn kennismaking met de afdeling radiologie in het MUMC was jij het eerste staflid waar ik aan werd voorgesteld door Prof. dr. J.M.A. van Engelshoven: 'met Regina kun jij wel zaken doen!'. En dat was ook zo. Jouw bevlogenheid voor de research is ook mij niet ontgaan. Inspirerende en soms felle research besprekingen waren kenmerkend voor jou. Het resultaat ligt hier nu voor je. De band die wij hebben opgebouwd is deels gebaseerd op eenzelfde drive om onze doelen te bereiken. Verder was er een niet onbelangrijke geografische band; onze domicilie in het mooie Eijsden. Ik ben blij met jou als promotor.

Prof. dr. B. Kremer, promotor. Beste Bernd, jouw enthousiasme voor het onderwerp van mijn thesis is voor mij inspirerend geweest. De expertise die jij hebt op het gebied van de hoofdhals oncologie heeft een positief effect gehad op het schrijven van de artikelen, wat ook heeft geresulteerd in goede en snelle publicaties. Zoals jij dat zelf noemde: "Es geht wie der Feuerwehr". Jouw ontspannen en open houding, interesse in de ander en hoffelijkheid maken dat het heel prettig samenwerken is geweest. Geheel voor de hand liggend dat jij mijn promotor bent.

Dr. J.W. Casselman, co-promotor. Beste Jan, na een periode met jou te hebben gewerkt in Brugge begrijp ik welke inspanning het vergt om wereldwijd te excelleren in de hoofdhals radiologie en dat in combinatie met het voeren van een algemene radiologische praktijk. Het resultaat hangt af van de inspanning. In Brugge hadden wij in mijn laatste twee dagen (en dus ook twee nachten!) iets meer 
dan 500 dossiers en bijbehorende MRI scans nagekeken. Snel opgeschreven leverde dat kort daarna een goede publicatie op. Belangrijker zijn jou twee gouden regels; houdt de clinici zoveel mogelijk te vriend, en wees nooit bang om kennis met anderen te delen want je wordt er zelf alleen maar beter van. Jij hebt jouw expertise van de hoofdhals radiologie met mij gedeeld, ik moet je zeggen dat we beide zijn gegroeid. Echter, het verschil tussen ons is wel even groot gebleven! Ik ben er trots op dat jij mijn co-promotor bent.

Dr. P.M. Nelemans, co-promotor. Beste Patty, jij wilde na mijn researchperiode niet de geschiedenis in gaan als "een rots in de branding". Voor wat betreft het epidemiologische deel en de statistische analyses was jij dat echter wel. Recht door zee, een kritische kijk op een onderzoek hebben zeker geresulteerd in een hogere kwaliteit van de artikelen en mede daardoor ook snelle acceptaties. Tijdens onze besprekingen dobberden wij vaak af naar andere onderwerpen zoals vakanties en forelvissen, ik denk met plezier terug aan onze bijeenkomsten.

De leden van de beoordelingscommissie, Prof. dr. C. Boetes, Prof. dr. J.O. Barentsz, Prof. dr. R.J. Baatenburg de Jong, Prof. dr. J.A. Castelijns, Prof. dr. P. Kessler en Prof. dr. Ph. Lambin ben ik zeer erkentelijk voor het beoordelen en goedkeuren van dit proefschrift.

Dr. L.M. Kingma, mijn opleider. Beste Lucas, dank voor de mogelijkheid om de opleiding radiologie te starten in het Westeinde ziekenhuis. Zonder dat zou ik nu niet promoveren op een radiologisch onderwerp. Jouw steun en inzet waren er ook om het laatste jaar van mijn opleiding in Nijmegen voort te zetten, dit vanwege 'een vriendin', niet lang daarna mijn vrouw.

Prof. dr. J.M.A. van Engelshoven. Beste Jos, dank dat je mij in Maastricht de mogelijkheid hebt geboden om de KNO radiologie uit te breiden en verdere invulling te geven. Daarnaast voor de mogelijkheid om promotieonderzoek te doen, het was opvallend de ruimte en vrijheid die jij daarbij gaf. Op afstand hield jij het proces in de gaten, zie hier nu het uiteindelijke resultaat.

Prof. dr. C. Boetes. Beste Carla, onze samenwerking gaat terug naar de periode dat ik mijn opleiding radiologie afrondde in Nijmegen. Al snel was er een vertrouwensband, deze werd jaren later weer voortgezet in Maastricht. Je weet dat ik jou zeer waardeer en dat ik probeer het contact te onderhouden. Dank voor het voorzitterschap van de beoordelingscommissie. 
De staf radiologie in het MUMC. Dank voor de samenwerking, ik heb veel mogelijkheden gehad om een bijdrage te leveren aan de vormgeving en verbetering van KNO radiologie. Daarnaast was er voldoende ruimte om mijzelf te ontwikkelen en om tijd te besteden aan promotieonderzoek. Ik kijk positief terug op mijn periode van 5 jaar die ik heb doorgebracht in Maastricht.

De staf KNO heelkunde in het MUMC, dank voor de samenwerking. Hierbij wil ik speciaal noemen Prof. dr. R.J. Stokroos. Beste Robert, met jou heb ik altijd een intensieve en plezierige samenwerking gehad, ook na mijn vertrek uit het Maastricht. Gesprekken over klinisch werk, research en (oude) auto's gingen altijd moeiteloos in elkaar over.

Ine Kengen, dank voor jouw inzet om mijn artikelen te voorzien van illustraties maar nog belangrijker het toevoegen van de eindeloze, steeds veranderende, referentielijsten. Dit getuigd pas van doorzettingsvermogen!

De dames van het secretariaat radiologie in Maastricht, Elfie, Christianne en Monique. Aan mijn eindeloze vragen en telefoontjes, vooral in de laatse fase van dit proefschrift, is ook een einde gekomen. Dank voor jullie ondersteuning.

De dames van het secretariaat KNO in Maastricht. Hoewel van een geheel andere afdeling zijn jullie naar mij altijd zeer welwillend geweest, dank daar voor.

Fons Kessels. Het heeft even geduurd voordat wij hetzelfde dachten over het begrip "onbekende primaire hoofdhalstumor bij bekende halskliermetastasen". Op het moment dat we dezelfde taal spraken was het STIR artikel af en al gepubliceerd.

De maatschap radiologie Isala Klinieken. Beste maten Aldert Taams, Boudewijn van Hasselt, Cor Holt, Corné van der Worp, Ernst Mulderije, Geert-Jan Waldman, Henk Boelhouwers, Jan Korte, Liesbeth Sijbrandij, Martijn Boomsma, Miranda van't Veer, Rolf van den Hoed, Zwenneke Flach (en Ben Zuidema). Jullie hebben geen last gehad van mijn promotie exercitie, hopelijk wel de lusten die het met zich mee brengt. Ik zie een positieve toekomst met de maatschap.

Mijn zusje, lieve Margaretha. Ik ben blij dat je aanwezig bent en de promotie meemaakt. Jij hebt je eigen weg gekozen en woont nu letterlijk op afstand, in gedachten is dat echter niet zo voor mij. We komen je snel opzoeken in het zuiden.

Ad en Hennie, mijn schoonouders. De vraag wanneer ik eindelijk eens afstudeer hoeft nu niet meer gesteld te worden, dat was overigens al in 2004. Jullie liefde, 
aandacht en steun aan ons gezin is enorm en onvoorwaardelijk. Daarvoor heb ik veel waardering en spreek dat nu dus ook uit. Ik hoop dat wij dit op eenzelfde manier kunnen doen voor onze kinderen.

Mijn paranimfen, Dennis den Hartog en Maarten Boot. Trouwe vrienden daar heb je slechts een klein aantal van, jullie zijn daar twee exemplaren van. Hoewel verschillend in karakter en professionele ontwikkeling zijn er toch ook een paar belangrijke overeenkomsten binnen onze vriendschappen te noemen: loyaliteit en eerlijkheid. Onze vriendschappen worden verder voornamelijk gekenmerkt door veel humor tijdens diners, vakanties of buitenlandse forelvistripjes. Ik ben blij dat jullie naast mij staan.

Mijn lieve ouders, Ruud en Lineke. Jullie zijn uiteraard voor een deel de reden van wie ik ben en waar ik nu sta. Afremmen van initiatieven was eerder aan de orde dan dat jullie mij moesten aansporen. Jullie steun aan mij tot dit moment met daarbij de vanzelfsprekendheid dat het allemaal goed komt is voor mij altijd een geruststellende gedachte geweest. De geografische afstand van de afgelopen twee decennia heeft gelukkig niet geleid tot een emotionele afstand, ik ben echter wel blij dat jullie weer in Nederland in de buurt komen wonen. Het is voor mij een mooi moment dat jullie de promotie vandaag allebei meemaken.

Lieve Margreet, wij zijn samen alweer tien jaar op weg. Een periode waar ik alleen maar positief op kan terug kijken. Samen vertrokken wij met onze eerste twee kinderen Sophie en Marijn naar het voor ons allebei onbekende Zuid-Limburg. In Eijsden werd ons gezin verrijkt met Merel. Ondanks dat wij goed konden aarden hebben wij toch het besluit genomen om verder te reizen terug naar de regio waar onze roots liggen. Jouw optimisme en enthousiasme zijn voor mij altijd een steun geweest. Verder zijn jouw vermogen om goed te kunnen relativeren, geduld en nuchtere kijk op de wereld een welkome aanvulling op mijn karakter. Samen hebben wij, zeker met onze drie kinderen, een mooi en rijk leven, dit is voor mij het allerbelangrijkste. Laten wij positief blijven kijken naar de toekomst en de reis samen voortzetten voor de rest van ons leven.

I love you. 


\section{Curriculum Vitae}

The author of this thesis was born in The Hague. After finishing high school at the Christelijk Lyceum Arnhem he started the training in physical therapy in Arnhem and Utrecht.

After three years, Medical School could be started in 1989 at the University of Amsterdam, for which he received the medical doctor degree in 1996. Subsequently, he started in the summer of 1996 a residency as Senior House Officer in Ear, Nose and Throat (ENT) surgery at the Kent County Ophthalmic \& Oral Hospital in Maidstone (Kent, England).

This was continued with a residency in ENT surgery at the Netherlands Cancer Institute/Antoni van Leeuwenhoek Hospital, Amsterdam.

The residency training in Radiology was started in 1998 at the Medical Center Haaglanden location Westeinde (Dr. L.M. Kingma) and finished as junior staff member at the University Medical Center Nijmegen (Dr. G.J. Jager).

In that last year he was trained in Neuroradiology and Head and Neck Radiology at the University Hospitals K.U. Leuven (Prof. dr. R. Hermans) and at the St. Jan Hospital, Bruges (Dr. J.W. Casselman) in Belgium.

From 2004 he was staff member at the Maastricht University Medical Center (MUMC) with the topic Head and Neck radiology.

The research program on MRI in lymph node imaging in head and neck cancer was started in 2006 resulting in this presented thesis.

In 2009 he continued his radiological career at the Isala Clinics in Zwolle.

He is married to Margreet Verveld, they have two daughters Sophie (2002) and Merel (2005), and a son Marijn (2003). 
$\longrightarrow$ 


\section{List of publications}

Diagnostic accuracy and additional value of diffusion weighted imaging for discrimination of malignant cervical lymph nodes in head and neck squamous cell carcinoma.

R.B.J. de Bondt, M.C. Hoeberigs, P.J. Nelemans, W.M.L.L.G. Deserno, C. Peutz-Kootstra, B. Kremer, R.G.H. Beets-Tan.

Neuroradiology. 2009 Mar;51(3):183-92.

Diffusion-weighted MR imaging of the head and neck.

Casselman JW, De Foer B, De Bondt BJ.

J Radiol. 2010 Mar;91(3):369-374.

Phenotypic manifestations and management of hyperostosis cranialis interna, a hereditary bone dysplasia affecting the calvaria and the skull base.

Waterval JJ, Stokroos RJ, Bauer NJ, De Bondt RB, Manni JJ.

Am J Med Genet A. 2010 Mar;152A(3):547-55.

Clinical impact of STIR MRI on staging and management in patients with cervical lymph node metastases of head and neck squamous cell carcinoma.

Bert-Jan de Bondt, Robert Stokroos, Jan W. Casselman, Jos M.A. van Engelshoven, Regina G.H. Beets-Tan, Fons G.H. Kessels.

Head Neck. 2009 Jul;31(7):928-37.

Facial nerve decompression via middle fossa approach for hyperostosis cranialis interna: a feasible therapeutic approach.

Waterval JJ, Stokroos RJ, De Bondt RB, Manni JJ.

J Laryngol Otol. 2009 Oct;123(10):1177-80.

Follow-up assessment of vestibular schwannomas: volume quantification versus two-dimensional measurements.

Van de Langenberg R, de Bondt BJ, Nelemans PJ, Baumert BG, Stokroos RJ. Neuroradiology. 2009 Aug;51(8):517-524.

Laryngeal tuberculosis presenting as a supraglottic carcinoma: a case report and review of the literature.

Smulders YE, De Bondt BJ, Lacko M, Hodge JA, Kross KW.

J Med Case Reports. 2009 Nov 20;3:9288. 
Synchronous bilateral epithelial-myoepithelial carcinoma of the parotid gland: case report and review of the literature.

van Tongeren J, Creytens DH, Meulemans EV, de Bondt RB, de Jong J, Manni JJ. Eur Arch Otorhinolaryngol. 2009 Sep;266(9):1495-500.

An unusual cause of transient neurologic deficits: compression of the carotid artery by a thyroid cystic nodule.

Braakman HM, Knippenberg SA, de Bondt BJ, Lodder J.

J Stroke Cerebrovasc Dis. 2010 Jan;19(1):73-4.

Destructive basal cell carcinoma in a patient with basal cell nevus syndrome and an interstitial deletion of chromosome 9q22.

Mosterd K, Sommer A, van Marion A, Lacko M, Herbergs J, de Bondt BJ, van Steensel MA, Kelleners-Smeets NW.

Dermatol Surg. 2009 Dec;35(12):2051-3.

Radiology quiz case 2. Metastasis from NSCLC in both IACs.

Van de Langenberg R, Stokroos R, de Bondt BJ.

Arch Otolaryngol Head Neck Surg. 2009 Jul;135(7):717-719.

Morphological MRI criteria improve the detection of lymph node metastases in head and neck squamous cell carcinoma: multivariate logistic regression analysis of MRI features of cervical lymph nodes.

R.B.J. de Bondt, P.J. Nelemans, F. Bakers, J.W. Casselman, C. Peutz-Kootstra, B. Kremer, P.A.M. Hofman, R.G.H. Beets-Tan.

Eur Radiol. 2009 Mar; 19(3):626-33.

Diagnose in beeld: een man met polssynchrone tinnitus.

R.B.J. de Bondt, T.K. Oei en J.J. Manni.

Nederlands Tijdschrift voor KNO-heelkunde. April 2008, 109.

Primary temporal bone secretory meningeoma presenting as chronic otitis media.

Marcelissen TA, de Bondt RB, Lammens M, Manni JJ.

Eur Arch Otorhinolaryngol 2008 Jul; 265(7) 843-7.

The fate of autologous hepatocytes transplanted into spleen, pancreas, mesentery, stomach, and small bowel in pigs.

Sosef MN, de Bondt RB, Bosma A, de Bruijn C, van Gulik TM.

Hepato-Pancreato-Biliary Association (Oxford) 2007;9(3):216-8. 
Detection of lymph nodes metastases in head and neck cancer: a meta-analysis comparing US, USgFNAC, CT and MR imaging.

R.B.J. de Bondt, P.J. Nelemans, P.A.M. Hofman, J.W. Casselman, B. Kremer, J.M.A. van Engelshoven, R.G.H. Beets-Tan.

European Journal of Radiology 64(2007) 266-272.

Permanent loss of cervical spinal cord function associated with the posterior approach.

Voermans NC, de Bondt RBJ, Crul BJ, Zwarts MJ, van Engelen BG.

Anesthesia \& Analgesia. 2006 Jan;102(1):330-1.

The "silent" progression of a secondary paranasal malignancy in a laryngectomized patient.

Bert Jan de Bondt, Robert Stokroos, Fons Balm and Jan Casselman.

European Journal of Radiology. June 2006; Volume 58 (3) 77-80.

Primary Non-Hodgkin Lymphoma of the cerebellopontine angle.

Stokroos RJ, de Bondt BJ, Hof J, Dings J, Casselman J. Laryngol Otol. 2007.

Persistent Trigeminal Artery (PTA) associated with Trigeminal Neuralgia; concept of a Neuro Vascular Conflict.

Bert-Jan de Bondt, Robert Stokroos, Jan Casselman.

Neuroradiology. 2006 Nov; Vol 49(1); 23-26.

Gerefereerde oorpijn: een belangrijk hoofdhals oncologisch signaal.

Bondt RBJ de, Balm AJM, Hilgers FJM, Tan IB.

NTVG 1998(1);142(32):1753-6.

\section{Book chapters}

Transplantation of autologous hepatocytes in pigs: 'a comparison of spleen, pancreas, mesentery and small bowel wall as implantation sites'.

Bondt RBJ de, Flendrig LM, Gulik TM van, Bosma A, Royen EA van, Chamuleau RAFM, Obertop H.

In: Proceedings Second World Congress of the International Hepato-PancreatoBiliary Association 1996; 1:33-6.

Lymph node imaging: present status and future developments.

In: Clinical Blood Pool MR Imaging (The Vasovist Product Monograph.

Eds. Tim Leiner, Mathias Goyen, Martin Rohrer, Stefan Schoenberg, Springer 2008). Hoofdstuk 16; 181-193. 
Chapters Diagnosis of the primary tumor and Diagnosis of cervical lymph node metastases.

In: Consensus development Hypopharynx carcinoma.

In: Revision Consensus development treatment Larynx carcinoma.

\section{Prizes and awards}

Diagnostic accuracy and additional value of diffusion weighted imaging for discrimination of malignant cervical lymph nodes in head and neck squamous cell carcinoma.

R.B.J. de Bondt, M.C. Hoeberigs, P.J. Nelemans, W.M.L.L.G. Deserno, C. Peutz-Kootstra, B. Kremer, R.G.H. Beets-Tan.

Best clinical study and presentation at the annual meeting of the European Society of Head and Neck Radiology (ESHNR), November 6-8 2008, Geneva, Switzerland.

Multivariate logistic analysis of MRI features of cervical lymph node: new MRI criteria improve the detection of lymph node metastases in head and neck squamous cell carcinoma.

R.B.J. de Bondt, P.J. Nelemans, F. Bakers, P.A.M. Hofman, B. Kremer, R.G.H. Beets-Tan.

Best study and presentation at the annual meeting of the Dutch Radiological Society (NVVR), September 27-28 2007, Rotterdam, The Netherlands.

Transplantation of autologous hepatocytes in pigs: a comparison of spleen, pancreas, mesentery and small bowel wall as implantation sites.

Bondt RBJ de, Flendrig LM, Gulik TM van, Bosma A, Royen EA van, Chamuleau RAFM, Obertop H.

Scientific Students award 1996 at the annual meeting of the Dutch Society of Gastro-enterology, March 21-22 1996, Veldhoven, The Netherlands.

Glaxo Wellcome Prize 1996, at the annual meeting of the German Society for Gastro-enterology, September 18-21 1996, Aachen, Germany. 